$$
\begin{gathered}
\text { UNIVERSIDADE DE SÃO PAULO } \\
\text { ESCOLA DE ENGENHARIA DE SÃO CARLOS } \\
\text { DEPARTAMENTO DE ENGENHARIA DE ESTRUTURAS }
\end{gathered}
$$

Gustavo Assis da Silva

Otimização topológica considerando incertezas com critério de falha em tensão

São Carlos 

Gustavo Assis da Silva

\title{
Otimização topológica considerando incertezas com critério de falha em tensão
}

\author{
VERSÃO CORRIGIDA \\ A versão original encontra-se na Escola de Engenharia de São Carlos
}

Tese apresentada ao Programa de PósGraduação em Engenharia de Estruturas da Escola de Engenharia de São Carlos, Universidade de São Paulo, como parte dos requisitos para a obtenção do título de Doutor em Ciências. Programa: Engenharia Civil (Estruturas).

Área de concentração: Estruturas

Orientador: Prof. Dr. André Teófilo Beck Coorientador: Prof. Dr. Eduardo Lenz Cardoso

\section{São Carlos}


AUTORIZO A REPRODUÇÃO TOTAL OU PARCIAL DESTE TRABALHO, POR QUALQUER MEIO CONVENCIONAL OU ELETRÔNICO, PARA FINS DE ESTUDO E PESQUISA, DESDE QUE CITADA A FONTE.

Ficha catalográfica elaborada pela Biblioteca Prof. Dr. Sérgio Rodrigues Fontes da EESC/USP com os dados inseridos pelo(a) autor(a).

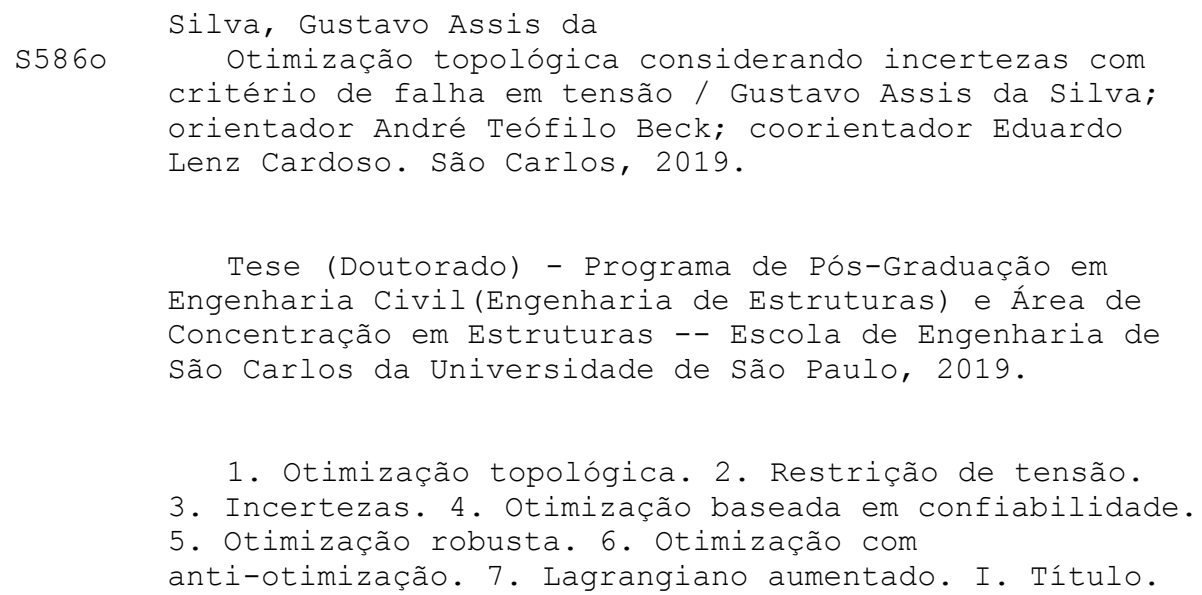

Eduardo Graziosi Silva - CRB - 8/8907 


\section{FOLHA DE JULGAMENTO}

Candidato: Engenheiro GUSTAVO ASSIS DA SILVA.

Título da tese: "Otimização topológica considerando incertezas com critério de falha em tensão".

Data da defesa: 19/02/2019.

\section{Comissão Julgadora:}

Prof. Associado André Teófilo Beck

\section{(Orientador)}

(Escola de Engenharia de São Carlos/EESC)

Prof. Titular Emílio Carlos Nelli Silva

(Escola Politécnica/EP-USP)

Prof. Dr. Eduardo Alberło Fancello

(Universidade Federal de Santa Catarina/UFSC)

Prof. Dr. André Jacomel Torii

(Universidade Federal da Paraíba/UFPB

Dr. Antônio André Novớny (Laboratório Nacional de Computação Científica/LNCC)

Coordenador do Programa de Pós-Graduação em Engenharia Civil (Engenharia de Estruturas):

Prof. Titular Humberło Breves Coda

Presidente da Comissão de Pós-Graduação:

Prof. Titular Luis Fernando Cosita Alberło
Resulfado:

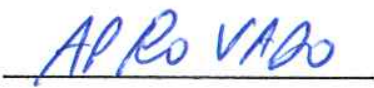

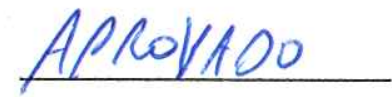

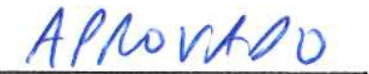





\section{AGRADECIMENTOS}

Inicialmente, gostaria de agradecer ao professor André Teófilo Beck pela orientação, pelas conversas, pelas trocas de ideias, por depositar confiança no meu trabalho, pelos ensinamentos que complementaram minha formação, e pelo suporte fornecido em diversas situações difíceis durante os anos de Doutorado.

Gostaria de agradecer ao professor Eduardo Lenz Cardoso por continuar acompanhando o desenvolvimento do meu trabalho durante estes anos de Doutorado, pelas trocas de ideias, e pela orientação fornecida em várias situações.

Gostaria de agradecer aos professores Humberto Breves Coda e Rodrigo Ribeiro Paccola pelos ensinamentos que complementaram minha formação. Agradeço ao professor Coda, também, pelas conversas, e pela orientação fornecida em diversas situações.

Agradeço ao professor Ole Sigmund por me receber na DTU (Technical University of Denmark), pela orientação, e por acreditar no meu trabalho.

Agradeço aos amigos que fiz durante estes anos de Doutorado pelos momentos de descontração.

Agradeço à minha família, que sempre incentivou os meus estudos.

Agradeço à minha esposa, Tássia Luize Soares, pelo apoio e incentivo prestados, em todos os momentos, e por me ajudar a seguir em frente e a não desistir, independentemente das dificuldades e desafios. Agradeço à Tássia por todos os momentos que passamos juntos.

Finalmente, gostaria de agradecer a FAPESP, processo número 2015/25199-0, pelo apoio financeiro, que foi fundamental para o desenvolvimento deste trabalho. 



\section{RESUMO}

SILVA, G. A. Otimização topológica considerando incertezas com critério de falha em tensão. 2019. 125p. Tese (Doutorado) - Escola de Engenharia de São Carlos, Universidade de São Paulo, São Carlos, 2019.

Hoje em dia, é amplamente reconhecido que o projeto de estruturas otimizadas deve ser robusto em relação a incertezas nas forças, geometria e propriedades do material. Entretanto, existem diversas alternativas para considerar tais incertezas em problemas de otimização estrutural. Esta tese apresenta quatro formulações para lidar com incertezas no problema de otimização topológica com restrição de tensão. As três primeiras são desenvolvidas para lidar com incertezas na intensidade e direção das forças aplicadas: 1) formulação robusta probabilística, onde substituem-se as restrições de tensão originais por uma soma ponderada entre os seus valores esperados e desvios padrão, obtidos por meio do método de perturbação de primeira ordem; 2) formulação baseada em confiabilidade, onde consideram-se restrições de tensão probabilísticas; o problema é formulado por meio de uma abordagem acoplada de primeira ordem; 3) formulação robusta não probabilística, onde considera-se o pior cenário possível para as restrições de tensão; o problema é formulado com uma abordagem acoplada de otimização com anti-otimização. A quarta formulação não segue o padrão das três primeiras; diferente das demais, esta é desenvolvida para lidar com incerteza uniforme de manufatura: 4) formulação robusta de três campos, onde três topologias são consideradas de forma simultânea durante o processo de otimização, de forma a simular possíveis imperfeições que possam ocorrer devido a erros de manufatura. As quatro abordagens são bastante diferentes na forma de lidar com as incertezas; no entanto, o procedimento de solução é o mesmo: a abordagem baseada em densidade é empregada na parametrização material, enquanto que o método do Lagrangiano aumentado é empregado para solucionar o problema resultante, de forma a lidar com o elevado número de restrições de tensão. Diversos exemplos são solucionados para mostrar a aplicabilidade das formulações propostas. Os exemplos são posteriormente verificados através da Simulação de Monte Carlo e comparados com os resultados determinísticos. Os resultados mostram que as estruturas obtidas com a abordagem tradicional determinística são extremamente sensíveis a incertezas. As formulações desenvolvidas nesta tese, por outro lado, mostraram-se alternativas válidas a formulação determinística, fornecendo resultados robustos e confiáveis na presença de incertezas.

Palavras-chave: Otimização topológica. Restrição de tensão. Incertezas. Otimização baseada em confiabilidade. Otimização robusta. Otimização com anti-otimização. Lagrangiano aumentado. 



\section{ABSTRACT}

\section{SILVA, G. A. Topology optimization under uncertainty with stress failure}

criterion. 2019. 125p. Tese (Doutorado) - Escola de Engenharia de São Carlos, Universidade de São Paulo, São Carlos, 2019.

It is nowadays widely acknowledged that optimal structural design should be robust with respect to the uncertainties in loads, geometry and material parameters. However, there are several alternatives to consider such uncertainties in structural optimization problems. This thesis addresses four formulations to handle uncertainties in topology optimization with stress constraint. The first three are developed to handle uncertainties in magnitude and direction of applied loads: 1) probabilistic robust formulation, where the original stress constraints are replaced by a weighted sum between their expectations and standard deviations; these are obtained by first-order perturbation approach; 2) reliability-based formulation, where probabilistic stress constraints are considered; the problem is formulated by a coupled first order approach; 3) non-probabilistic robust formulation, where the worstcase scenario for the stress constraints is considered; the problem is formulated by a coupled approach called optimization with anti-optimization. The fourth formulation is quite different from the first three; it is developed to handle uniform boundary variation: 4) three-field robust approach, where three topologies are simultaneously considered during the optimization process, in order to simulate imperfections which may occur due to manufacturing errors. These four formulations are quite different in handling with uncertainties; however, the solution procedure is the same: the density approach is employed to material parameterization, while the augmented Lagrangian method is employed to solve the resulting problem, in order to handle the large number of stress constraints. Several examples are solved to demonstrate applicability of proposed formulations. Numerical examples are further verified via Monte Carlo Simulation and compared to deterministic results. The results show that the structures obtained with traditional deterministic formulation are extremely sensitive to uncertainties. On the other hand, the formulations developed in this thesis are shown to be valid alternatives to the deterministic formulation, providing robust and reliable results in the presence of uncertainties.

Keywords: Topology optimization. Stress constraint. Uncertainties. Reliability-based optimization. Robust optimization. Optimization with anti-optimization. Augmented Lagrangian. 



\section{LISTA DE FIGURAS}

Figura 1 - Otimização topológica - ilustração: (a) domínio de projeto e condições de contorno; (b) topologia otimizada. . . . . . . . . . . . . .

Figura 2 - Critério de falha em tensão na otimização topológica: (a) domínio de projeto e condições de contorno; (b) estrutura de mínima flexibilidade; (c) estrutura de mínimo volume dado critério de falha em tensão. . . . 22

Figura 3 - Otimização topológica sob incerteza: (a) problema determinístico; (b) solução do problema determinístico com critério de falha em tensão; (c) pós-processamento da tensão máxima normalizada $\sigma_{e q}^{\max } / \sigma_{y}$ para $\theta \in[0,2 \pi] \mathrm{rad} ;(\mathrm{d})$ problema robusto; (e) solução do problema robusto; (f) pós-processamento para $\theta \in[0,2 \pi] \mathrm{rad}$.

Figura 4 - Problema de otimização topológica por meio da distribuição de material isotrópico. . . . . . . . . . . . . . . . . 27

Figura 5 - Otimização topológica baseada em densidade - ilustração: (a) problema contínuo; (b) problema discretizado; (c) topologia otimizada no domínio discretizado; (d) topologia pós-processada por meio de técnica de extração de contorno. . . . . . . . . . . . . . . . . . . . . .

Figura 6 - Instabilidade de tabuleiro e dependência da malha: (a) exemplo de instabilidade de tabuleiro; (b) solução para discretização com 600 elementos; (c) solução para discretização com 5400 elementos.

Figura 7 - Topologias otimizadas considerando filtro de densidades: (a) 300; (b) 1200; (c) 4800; (d) 19200 elementos. . . . . . . . . . . . . . . . . . . 36

Figura 8 - Distribuição de tensão em estrutura com contorno irregular: (a) estrutura; (b) primeiras tensões principais avaliadas no centroide de cada elemento dado um carregamento de tensão uniaxial na barra. . . . . . . . 54

Figura 9 - Problema da chapa com furo circular: (a) domínio completo e condições de contorno; (b) 1/4 do domínio após aplicação das condições de simetria; (c) modelo discretizado com o MEF baseado em pixel. . . . . . . . . . 66

Figura 10 - Tensão máxima $\sigma_{11}$ calculada com três malhas distintas e comparada com a solução de referência ( $\cong 3$, linha cheia). O tamanho das legendas horizontais representa o tamanho dos elementos (lado dos quadrados). . 67

Figura 11 - Procedimento de filtragem e projeção utilizado: (a) campo de projeto; (b) campo filtrado; (c) campo projetado para $\delta \rightarrow \infty$; (d) campo projetado para $\delta=32$. Os campos projetados são avaliados para cinco valores distintos de $\eta$, da esquerda para direita: $0.1,0.25,0.5,0.75$ e $0.9 \ldots$. . 68

Figura 12 - Derivadas dos campos projetados em relação à $x$, para $\delta=32$ (Figura 11 (d)) e cinco valores diferentes de $\eta$. 
Figura 13 - Ilustração do comportamento assintótico da Equação (5.24), para $R=1.70$

Figura 14 - Gráficos de tensão para diferentes valores de $\varepsilon$ e tamanhos de malha. Cada gráfico foi gerado para quatro valores diferentes de $\delta$ e comparado com a solução de referência ( $\cong 3)$. O tamanho das linhas horizontais indica o tamanho dos elementos na malha. . . . . . . . . . . . . 72

Figura 15 - Fluxograma simplificado com a abordagem de solução proposta. . . . . 77

Figura 16 - Domínios em forma de L considerados, com dimensões e condições de contorno. . . . . . . . . . . . . . . . . 82

Figura 17 - Escala de cores utilizada para ilustração de topologias. Branco representa a fase vazia $(\bar{\rho}=0)$ e preto a fase sólida $(\bar{\rho}=1)$ da topologia.

Figura 18 - Escala de cores utilizada para ilustração de tensões de von Mises e índices de confiabilidade pós-processados. Nos gráficos de tensão, vermelho representa a tensão máxima normalizada $(\cong 1)$ e azul a tensão mínima normalizada $(\cong 0)$. Nos gráficos de índices de confiabilidade, vermelho representa o índice de confiabilidade mínimo e azul o máximo. . . . . .

Figura 19 - Topologias otimizadas e respectivas tensões equivalentes de von Mises para diferentes discretizações. Da esquerda para direita: $N_{e}=57600$ $(300 \times 300) ; N_{e}=102400(400 \times 400) ; N_{e}=160000(500 \times 500) ;$ $N_{e}=230400(600 \times 600)$. Problema $($ L1) determinístico.

Figura 20 - Topologias otimizadas e respectivas tensões equivalentes de von Mises para diferentes discretizações. Da esquerda para direita: $N_{e}=57600$ $(300 \times 300) ; N_{e}=102400(400 \times 400) ; N_{e}=160000(500 \times 500) ;$ $N_{e}=230400(600 \times 600)$. Problema $($ L2) determinístico. . . . . . . . 84

Figura 21 - Históricos de convergência associados aos resultados obtidos para as malhas mais refinadas $\left(N_{e}=230400\right)$. Problemas: (L1), esquerda; e (L2), direita. . . . . . . . . . . . . . . . . . . 85

Figura 22 - Problemas considerados na subseção 7.1.2: (a) problema de domínio retangular sob duas forças incertas; (b) problema (L1) sob uma força incerta e uma força determinística; com dimensões e condições de contorno. 87

Figura 23 - Resultados do problema de domínio retangular: topologias (esquerda); tensões de von Mises (centro); índices de confiabilidade pós-processados com SMC (direita). As tensões ilustradas são as próprias medidas de tensão adotadas em cada problema. Os volumes estruturais, tensões máximas e índices de confiabilidade mínimos são mostrados abaixo de cada figura. . . . . . . . . . . . . . . . . . . . . . 89

Figura 24 - Resultados do problema (L1) sob uma força incerta: topologias (esquerda); tensões de von Mises (centro); índices de confiabilidade pósprocessados com SMC (direita). As tensões ilustradas são as próprias medidas de tensão adotadas em cada problema. . . . . . . . . . . . . . 92 
Figura 25 - Topologias otimizadas obtidas como solução do problema (L1) considerando, da direita para a esquerda, otimização com: 1 força determinística; 4 casos de força determinísticos; 8 casos de força determinísticos; anti-otimização com $\theta \in[0,2 \pi]$ rad. . . . . . . . . . . . . . . 95

Figura 26 - Topologias otimizadas obtidas como solução do problema (L2) considerando, da direita para a esquerda, otimização com: 1 força determinística; 4 casos de força determinísticos; 8 casos de força determinísticos; anti-otimização com $\theta \in[0,2 \pi]$ rad. . . . . . . . . . . . . . . 96

Figura 27 - Problema (L2) considerado nesta subseção. Dimensões em metro e força em Newton. . . . . . . . . . . . . . . . . . . . . . . .

Figura 28 - Topologias e respectivas tensões equivalentes de von Mises para estruturas otimizadas erodida (esquerda), intermediária (centro) e dilatada (direita) . . . . . . . . . . . . . . . . . . . 9 98

Figura 29 - Procedimento empregado para extração de contorno suave das topologias otimizadas. . . . . . . . . . . . . . . . . . . 100

Figura 30 - Tensões de von Mises máximas pós-processadas. Resultados referentes às estruturas erodida, intermediária e dilatada, considerando o modelo ajustado ao corpo, são indicados por círculos.

Figura 31 - Curvas de nível, estrutura erodida: (a) considerando o furo; (b) desconsiderando o furo; e respectivas tensões equivalentes de von Mises resultantes de modelos ajustados ao corpo: (c) e (d) . . . . . . . . . . . 102

Figura 32 - Tensões equivalentes de von Mises obtidas para os modelos baseado em pixel (esquerda) e ajustado ao corpo (direita), para $\eta=0.5$. . . . . . 103

Figura 33 - Topologias intermediárias (robustas) e detalhes dos cantos reentrantes com densidades filtradas e curvas de nível consideradas no pósprocessamento, para $N_{e}=25600$ (esquerda) e $N_{e}=102400$ (direita). . 104

Figura 34 - Tensões de von Mises máximas pós-processadas, para $N_{e}=25600$. Resultados referentes às estruturas erodida, intermediária e dilatada, considerando o modelo ajustado ao corpo, são indicados por círculos. 105

Figura 35 - Problema de domínio com furo circular. . . . . . . . . . . . . . . 107

Figura 36 - Topologias (esquerda) e respectivas curvas de nível (direita). Tensões obtidas com modelos baseados em pixel (esquerda) e ajustados ao corpo (direita). Problema de domínio com furo circular. . . . . . . . . . . 108

Figura 37 - Tensões de von Mises máximas pós-processadas, problema de domínio com furo circular. Resultados referentes às estruturas erodida, intermediária e dilatada, considerando o modelo ajustado ao corpo, são indicados por círculos. . . . . . . . . . . . . . . . . . 109

Figura 38 - Problema de domínio com corte triangular. . . . . . . . . . . . . . . 109 
Figura 39 - Topologias (linha 1), respectivas curvas de nível (linha 2), tensões obtidas com modelos baseados em pixel (linha 3) e ajustados ao corpo (linha 4). Problema de domínio com corte triangular. . . . . . . . . . . 110

Figura 40 - Tensões de von Mises máximas pós-processadas, problema de domínio com corte triangular. Resultados referentes às estruturas erodida, intermediária e dilatada, considerando o modelo ajustado ao corpo, são indicados por círculos. . . . . . . . . . . . . . . . . . 111 


\section{LISTA DE TABELAS}

Tabela 1 - Número de iterações até a convergência, problemas determinísticos (L1)

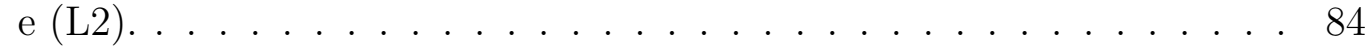

Tabela 2 - Volumes estruturais, índices de confiabilidade mínimos, probabilidades de falha máximas e número de iterações até a convergência. Problema de domínio retangular. . . . . . . . . . . . . . . . . . . . . 88

Tabela 3 - Volumes estruturais, índices de confiabilidade mínimos, probabilidades de falha máximas e número de iterações até a convergência. Problema (L1) sob uma força incerta. . . . . . . . . . . . . . . . . . . . . 93

Tabela 4 - Número de iterações até a convergência, problemas determinísticos e robustos $(\mathrm{L} 1)$ e $(\mathrm{L} 2) \ldots \ldots$. . . . . . . . . . . . . . . . . . . 97 



\section{LISTA DE ABREVIATURAS E SIGLAS}

HMV Hybrid Mean Value

IVE Interior Value Extrapolation

MEF Método dos Elementos Finitos

MEMS MicroElectroMechanical Systems

PMA Performance Measure Approach

RAMP Rational Approximation of Material Properties

SIMP Solid Isotropic Material with Penalization

SMC Simulação de Monte Carlo

SORA Sequential Optimization and Reliability Assessment 



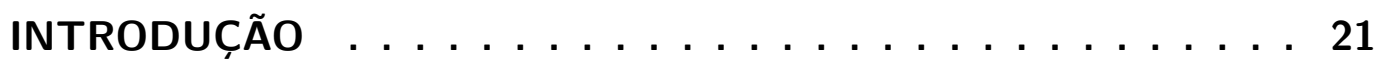

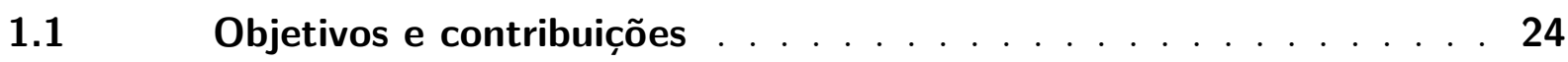

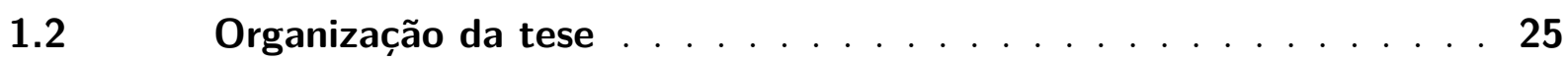

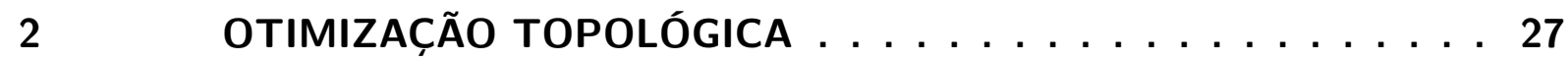

$2.1 \quad$ Dificuldades associadas $\ldots \ldots \ldots \ldots \ldots \ldots \ldots$

$2.1 .1 \quad$ Dependência da malha . . . . . . . . . . . . . . 32

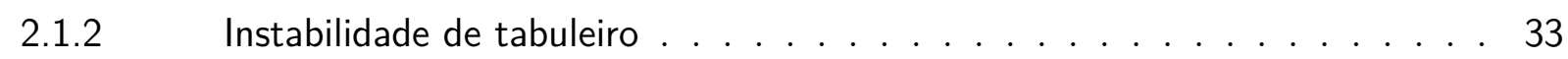

$2.2 \quad$ Métodos de restrição do espaço de solução . . . . . . . . . . . 34

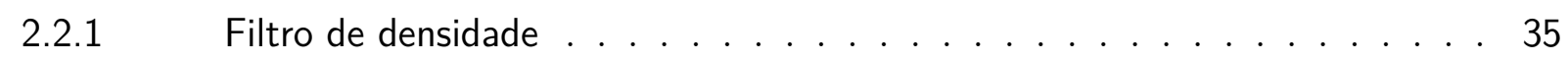

$2.2 .2 \quad$ Métodos de projeção . . . . . . . . . . . . . . 36

2.2.2.1 Projeção de dilatação . . . . . . . . . . . . . . . . . . 36

2.2.2.2 Projeção parametrizada . . . . . . . . . . . . . . . . . . . 37

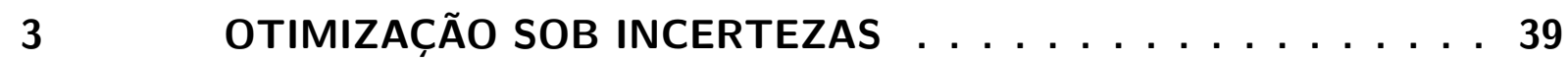

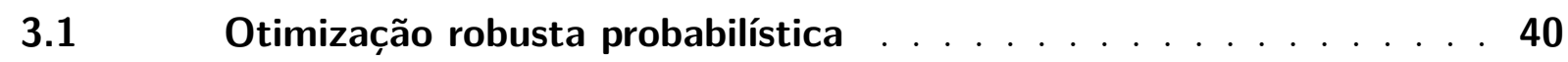

$3.2 \quad$ Otimização baseada em confiabilidade . . . . . . . . . . . 43

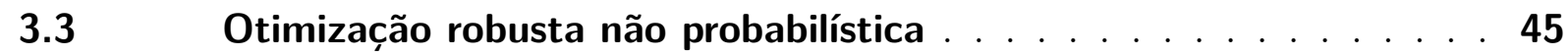

4 OTIMIZAÇÃO TOPOLÓGICA COM RESTRIÇÃO DE TENSÃO . . 49

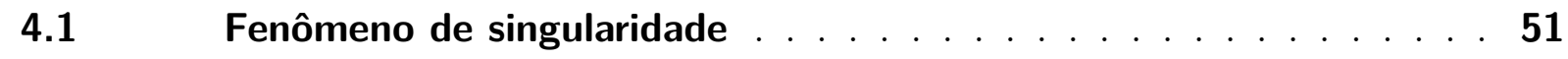

$4.2 \quad$ Natureza local do critério de falha em tensão . . . . . . . . . . 52

$4.3 \quad$ Cálculo da tensão no contorno irregular . . . . . . . . . . . . . 54

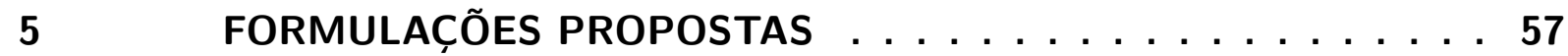

5.1 Minimização de volume com restrição de tensão e incerteza nas

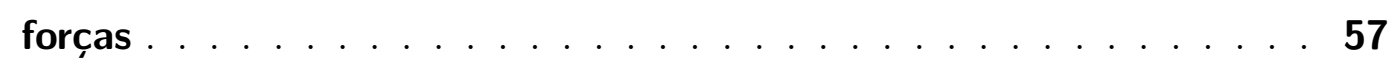

$5.1 .1 \quad$ Formulação robusta probabilística $\ldots \ldots \ldots \ldots \ldots$

$5.1 .2 \quad$ Formulação baseada em confiabilidade . . . . . . . . . . . 60

5.1.3 Formulação robusta não probabilística . . . . . . . . . . . . . 62

5.2 Minimização de volume com restrição de tensão e incerteza de manufatura . . . . . . . . . . . . . . . . . 64

5.2.1 Análise: chapa com furo circular . . . . . . . . . . . . . . 65

5.2.2 Limitando a nitidez dos campos projetados . . . . . . . . . . . 67

5.2.3 Escolhendo um parâmetro de interpolação de tensão adequado . . . . . . 70

6 SOLUÇÃO DO PROBLEMA DE OTIMIZAÇÃO $\ldots \ldots \ldots \ldots$ 
$6.1 \quad$ Método do Lagrangiano aumentado . . . . . . . . . . . . . 75

6.1.1 Problemas com incerteza nas forças . . . . . . . . . . . . . . . 77

6.1.2 Problema com incerteza de manufatura . . . . . . . . . . . . 78

6.2 Solução dos subproblemas de otimização . . . . . . . . . . . . 78

7 RESULTADOS NUMÉRICOS E DISCUSSÕES . . . . . . . . 81

7.1 Problema com restrição de tensão sob incerteza nas forças . . . . 81

7.1.1 Problema determinístico: estudo de dependência da malha . . . . . . . . 81

7.1.2 Problema sob incerteza na intensidade das forças . . . . . . . . . 86

7.1.2.1 Problema de domínio retangular sob duas forças incertas . . . . . . . . 87

7.1.2.2 Problema (L1) sob uma força incerta . . . . . . . . . . . . . . 90

7.1.3 Problema sob incerteza na direção das forças . . . . . . . . . . . . . 93

7.2 Problema com restrição de tensão sob incerteza de manufatura . . 97

7.2 .1 Influência do tamanho da malha . . . . . . . . . . . . . . . . 104

7.2.2 Resultados numéricos complementares . . . . . . . . . . . . . . 106

7.2.2.1 Problema de domínio com furo circular . . . . . . . . . . . . . 106

7.2.2.2 Problema de domínio com corte triangular . . . . . . . . . . . . . . 108

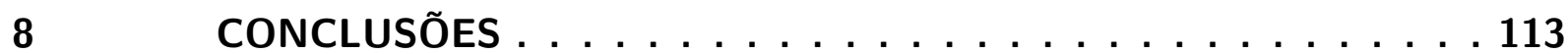

8.1 Conclusões específicas: problemas sob incerteza nas forças . . . . 113

8.2 Conclusões específicas: problemas sob incerteza de manufatura . . 114

$8.3 \quad$ Sugestões para trabalhos futuros . . . . . . . . . . . . . . . . 114

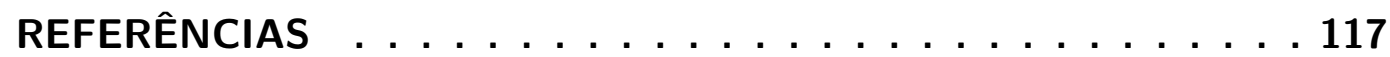




\section{INTRODUÇÃO}

Otimização topológica é uma importante ferramenta que é frequentemente utilizada no projeto de estruturas de alto desempenho (BENDSØE; SIGMUND, 2003). Dado um problema de projeto estrutural, a otimização topológica de estruturas contínuas consiste na distribuição de material dentro de um domínio fixo de projeto, com o intuito de minimizar/maximizar uma medida de desempenho, tal que o conjunto de restrições de projeto seja satisfeito (BENDSØE; SIGMUND, 2003), Figura 1.

Figura 1: Otimização topológica - ilustração: (a) domínio de projeto e condições de contorno; (b) topologia otimizada.

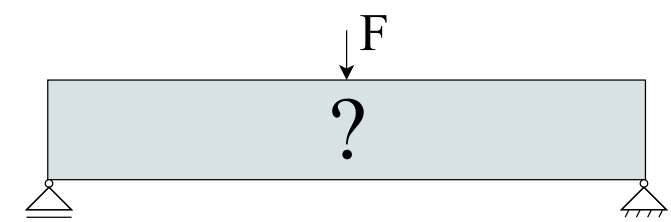

(a)
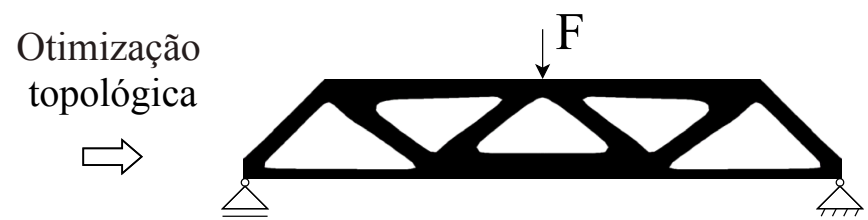

(b)

Fonte: adaptado de Bendsøe e Sigmund (2003).

Desde o trabalho pioneiro de Bendsøe e Kikuchi (1988), onde a abordagem de distribuição de material foi pela primeira vez apresentada como uma ferramenta computacional sistemática para a otimização topológica de estruturas, a área de otimização topológica em meios contínuos tem sido alvo de intensa pesquisa na literatura (SIGMUND; MAUTE, 2013). O amadurecimento da literatura em técnicas de otimização topológica e a disponibilidade de melhores recursos computacionais permitiram a inclusão de requisitos de projeto mais realísticos na otimização topológica de estruturas contínuas, como a inclusão do critério de falha em tensão (DUYSINX; BENDSØE, 1998; LE et al., 2010; PEREIRA; FANCELLO; BARCELLOS, 2004) e a consideração de incertezas durante a etapa de projeto (JUNG; CHO, 2004; MAUTE; FRANGOPOL, 2003; WANG; LAZAROV; SIGMUND, 2011), requisitos estes diretamente relacionados com esta tese.

A inclusão do critério de falha em tensão é particularmente importante quando se deseja evitar concentrações ou singularidades de tensão (LE et al., 2010). Originalmente, na literatura, a otimização topológica foi empregada no projeto de estruturas de mínima flexibilidade (máxima rigidez), dada uma quantidade de material estabelecida a priori (restrição de volume) (BENDSØE; SIGMUND, 2003). O projeto baseado em tensão surgiu como uma alternativa e um complemento a formulação de mínima flexibilidade, permitindo a inclusão de critérios de falha mais realísticos durante o projeto estrutural. A Figura 2 ilustra a diferença entre ambas as formulações; observa-se que a consideração de um critério de falha em tensão permite a obtenção de uma estrutura com canto arredondado, evitando a singularidade de tensão do domínio de projeto. 
Figura 2: Critério de falha em tensão na otimização topológica: (a) domínio de projeto e condições de contorno; (b) estrutura de mínima flexibilidade; (c) estrutura de mínimo volume dado critério de falha em tensão.

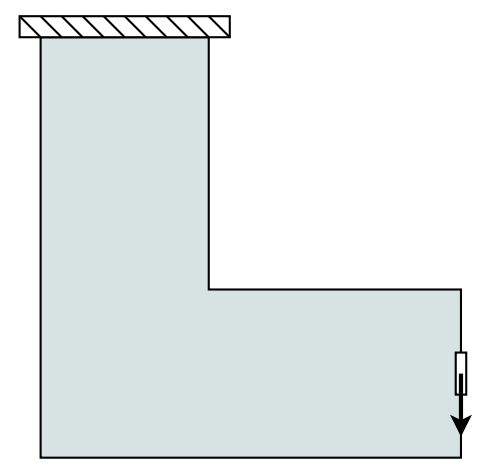

(a)

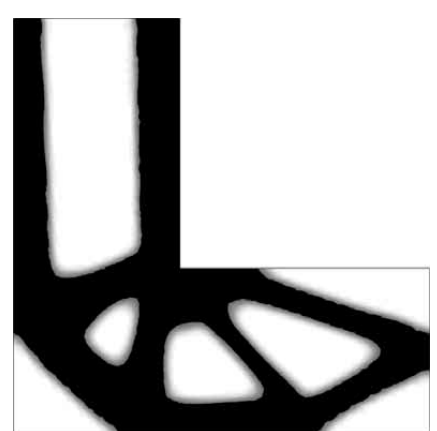

(b)

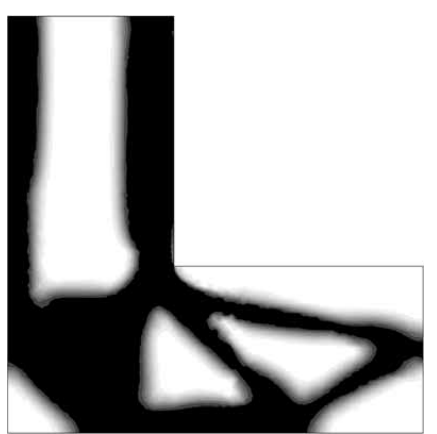

(c)

Fonte: adaptado de Pereira, Fancello e Barcellos (2004).

A consideração de incertezas na etapa de projeto, por sua vez, é importante no projeto de estruturas robustas e confiáveis, que não sejam suscetíveis a perturbações em parâmetros que afetam a resposta estrutural (BEYER; SENDHOFF, 2007). Conforme mostrado em Silva, Cardoso e Beck (2018), a otimização topológica determinística fornece soluções extremamente instáveis, Figura 3 (b) e (c), não sendo adequada na solução de problemas de otimização topológica sujeitos a incertezas. A otimização topológica considerando incertezas surgiu como uma alternativa a abordagem determinística tradicional, de forma a lidar com as incertezas que afetam a resposta estrutural durante o procedimento de otimização e fornecer estruturas confiáveis e que não sejam suscetíveis a perturbações nos parâmetros do modelo, Figura 3 (e) e (f).

Esta tese visa o estudo de problemas de otimização topológica de estruturas contínuas sujeitos, de forma simultânea, tanto ao critério de falha em tensão quanto a incertezas. Apesar de já existirem trabalhos propondo formulações com critério de falha em tensão, e trabalhos propondo formulações que lidam com as incertezas do problema estrutural, trabalhos com formulações unificadas que lidam, de forma simultânea, com o critério de falha em tensão e com incertezas, são escassos na literatura. Formulações unificadas têm sido propostas apenas recentemente, devido a sua elevada complexidade. Uma revisão cuidadosa e abrangente da literatura mostrou cinco trabalhos recentes propondo formulações unificadas (HOLMBERG; THORE; KLARBRING, 2017; LUO et al., 2014; SANTOS; TORII; NOVOTNY, 2018; SILVA; CARDOSO, 2017; THORE; HOLMBERG; KLARBRING, 2017), descritos brevemente nos próximos parágrafos.

Silva e Cardoso (2017) propõem uma formulação probabilística robusta para solucionar o problema baseado em tensão sujeito a incerteza nas propriedades do material. Propõe-se a quantificação de incertezas por meio da técnica de perturbação de primeira ordem. Neste trabalho, as restrições de tensão são escritas como uma soma ponderada do 
Figura 3: Otimização topológica sob incerteza: (a) problema determinístico; (b) solução do problema determinístico com critério de falha em tensão; (c) pós-processamento da tensão máxima normalizada $\sigma_{e q}^{\max } / \sigma_{y}$ para $\theta \in[0,2 \pi] \mathrm{rad} ;(\mathrm{d})$ problema robusto; (e) solução do problema robusto; (f) pós-processamento para $\theta \in$ $[0,2 \pi] \mathrm{rad}$.

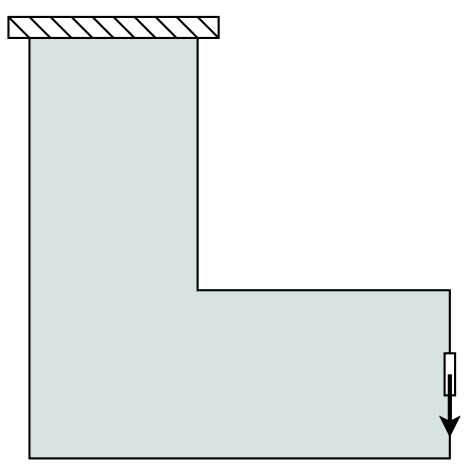

(a)

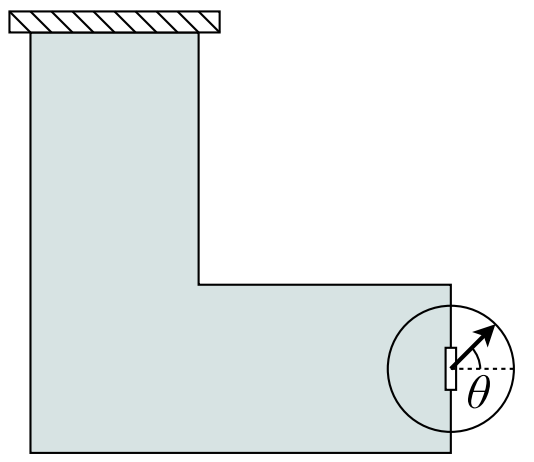

(d)

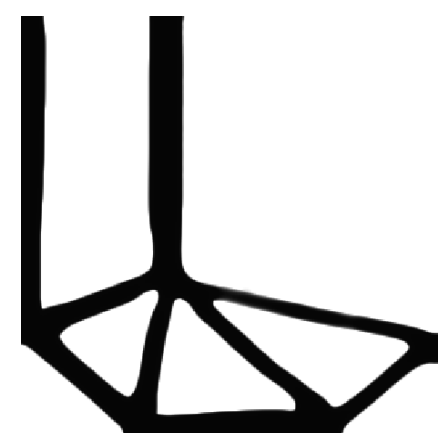

(b)

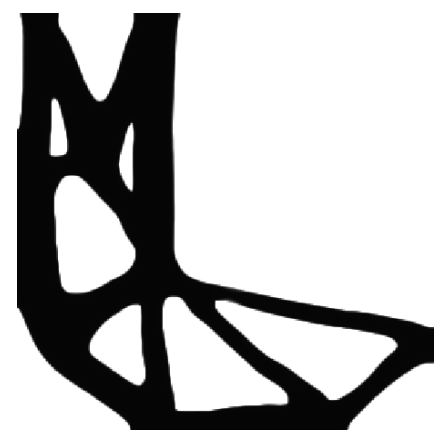

(e)

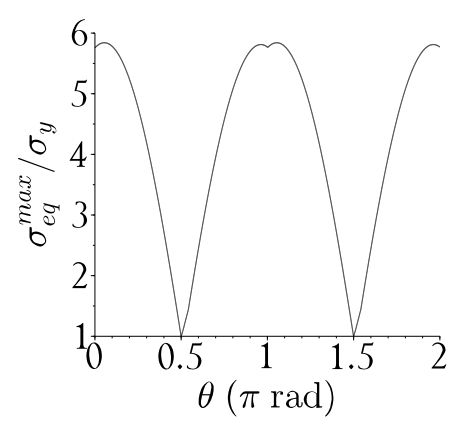

(c)

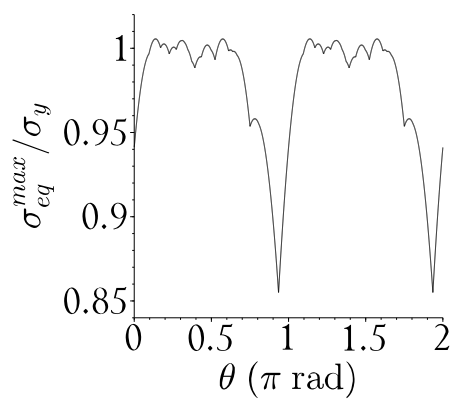

(f)

Fonte: adaptado de Silva, Cardoso e Beck (2018).

seu valor esperado e do seu desvio padrão.

Luo et al. (2014) propõem uma formulação baseada em confiabilidade para solucionar problemas baseados em tensão sob incerteza nas forças externas e propriedades do material. Uma abordagem acoplada de primeira ordem é empregada para formular o problema baseado em confiabilidade e uma técnica de agregação é utilizada para lidar com o elevado número de restrições de tensão. Neste trabalho, sugere-se que métodos de relaxação tradicionais não são suficientes para evitar o fenômeno de singularidade quando incertezas são consideradas, de forma que uma relaxação adicional no índice de confiabilidade alvo é necessária para evitar que o algoritmo obtenha topologias não ótimas.

Santos, Torii e Novotny (2018) propõem uma formulação baseada em confiabilidade para lidar com incertezas nas forças externas e no limite de tensão no problema de otimização topológica. O problema é formulado considerando uma abordagem desacoplada de primeira ordem, onde efetua-se, de forma sequencial, uma otimização determinística e uma análise de confiabilidade inversa (SORA - Sequential Optimization and Reliability Assessment (DU; CHEN, 2004)), até que a obtenção de uma estrutura otimizada que 
respeite a restrição probabilística sobre a tensão máxima seja obtida.

Holmberg, Thore e Klarbring (2017) propõem uma formulação robusta não probabilística para solucionar problemas baseados em tensão considerando carregamentos incertos-mas-limitados. Os autores propõem uma abordagem baseada em ciclo para a solução do problema sob incerteza, onde efetuam, de forma sequencial, uma otimização determinística e uma análise de pior cenário, até a obtenção de uma estrutura que seja robusta em relação às incertezas nas forças aplicadas.

Thore, Holmberg e Klarbring (2017) propõem uma formulação robusta não probabilística onde considera-se que a função objetivo e as restrições de projeto são funções quadráticas dos carregamentos aplicados, permitindo a utilização de um modelo elipsoidal na avaliação do pior cenário das tensões, dados carregamentos incertos-mas-limitados. Esta formulação permite a solução de problemas sujeitos a uma restrição sobre a norma Euclidiana das tensões.

Apesar destas contribuições apresentarem formulações e métodos para a solução de problemas baseados em tensão considerando incertezas, muita pesquisa ainda se faz necessária para o desenvolvimento de formulações e métodos complementares. Além disso, a área em questão é extremamente desafiadora, de forma que muita pesquisa ainda é necessária para definir quais os métodos mais robustos, práticos e sistemáticos na solução destes problemas. Conforme descrito a seguir, esta tese propõe diversas formulações e métodos que podem ser utilizados tanto como um complemento como uma alternativa às formulações unificadas previamente propostas.

\subsection{Objetivos e contribuições}

Este trabalho visa o estudo do problema de minimização de volume com restrição local de tensão considerando incertezas. Os objetivos e contribuições desta tese são descritos a seguir.

1. Propor uma formulação:

- Robusta probabilística, para lidar com incertezas na intensidade e direção das forças aplicadas. Esta formulação é descrita em detalhes em Silva, Beck e Cardoso (2018), que consiste em um trabalho recente do autor e fruto desta tese, e também na subseção 5.1.1;

- Baseada em confiabilidade (probabilística), para lidar com incertezas na intensidade e direção das forças aplicadas. Esta formulação é descrita em detalhes em Silva e Beck (2018), que consiste em um trabalho recente do autor e fruto desta tese, e também na subseção 5.1.2; 
- Robusta não probabilística, para lidar com incertezas na intensidade e direção das forças aplicadas. Esta formulação é descrita em detalhes em Silva, Cardoso e Beck (2018), que consiste em um trabalho recente do autor e fruto desta tese, e também na subseção 5.1.3;

- Robusta não probabilística, para lidar com incerteza uniforme de manufatura. Esta formulação é descrita em detalhes em Silva, Beck e Sigmund (2019), que consiste em um trabalho recente do autor e fruto desta tese, e também na subseção 5.2

2. Propor um procedimento de solução baseado no método do Lagrangiano aumentado, que possa ser utilizado para solução de problemas determinísticos ou sob incerteza, sujeitos a um elevado número de restrições de tensão. Seção 6;

3. Solucionar exemplos clássicos da literatura e comparar os resultados obtidos com os resultados determinísticos. Seção 7;

4. Validar os resultados obtidos por meio de pós-processamento adequado. Seção 7.

\subsection{Organização da tese}

De forma a facilitar ao leitor, dividimos esta tese em 5 partes, cada uma contendo uma ou mais seções, conforme descrito a seguir:

1. Contextualização e revisão bibliográfica, seções 2,3 e 4 .

2. Formulações propostas, seção 5 .

3. Procedimento de solução proposto, seção 6 .

4. Resultados e discussões, seção 7.

5. Conclusões e sugestões para trabalhos futuros, seção 8. 



\section{OTIMIZAÇÃO TOPOLÓGICA}

Neste trabalho, aborda-se o problema estrutural de otimização topológica de distribuição de material isotrópico. Considerando um domínio fixo de projeto $\Omega \in \mathbb{R}^{d}$, onde $d=2$ ou 3 (ilustrado na Figura 4 para $d=2$ ), o objetivo da otimização topológica consiste em obter a distribuição de material que minimize/maximize o desempenho da estrutura, ao mesmo tempo em que os requisitos de projeto sejam satisfeitos (BENDSØE; SIGMUND, 2003).

Figura 4: Problema de otimização topológica por meio da distribuição de material isotrópico.

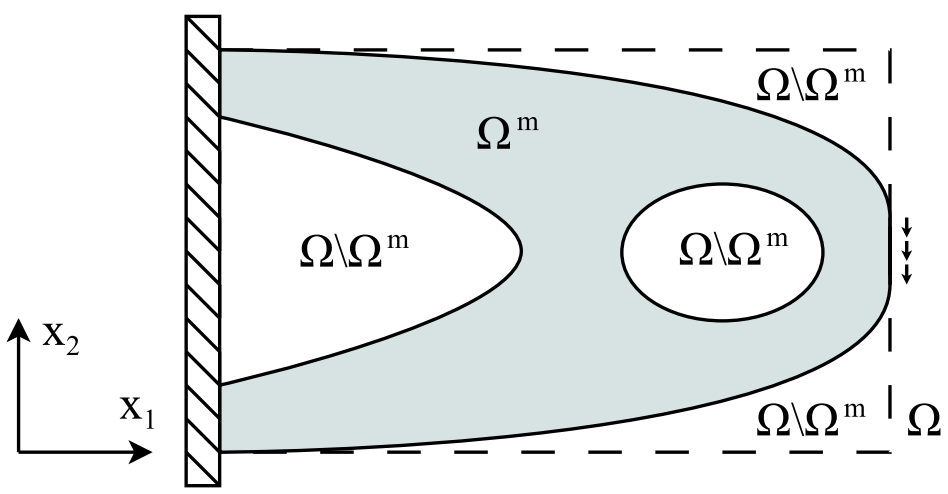

Fonte: produção do próprio autor.

Um problema de otimização topológica de distribuição de material isotrópico é idealmente formulado por meio de uma parametrização discreta $\Theta(\mathbf{x})$, que descreve o tensor constitutivo no domínio de projeto,

$$
C_{i j k l}(\mathbf{x})=\Theta(\mathbf{x}) C_{i j k l}^{b}
$$

onde $C_{i j k l}(\mathbf{x})$ é o tensor constitutivo do material, que depende da sua posição no domínio de projeto $\mathbf{x} \in \Omega$, e $C_{i j k l}^{b}$ é o tensor constitutivo do material base (BENDSØE; SIGMUND, 1999). A parametrização discreta é escrita como

$$
\Theta(\mathbf{x})=\left\{\begin{array}{ll}
1 & \text { se } \mathbf{x} \in \Omega^{m} \\
0 & \text { se } \mathbf{x} \in \Omega \backslash \Omega^{m}
\end{array},\right.
$$

onde $\Omega^{m}$ é a fase sólida e $\Omega \backslash \Omega^{m}$ a fase vazia da topologia, Figura 4 .

Pode-se escrever um problema genérico de otimização topológica de distribuição de 
material isotrópico como

$$
\begin{array}{lll}
\underset{\Theta(\mathbf{x})}{\operatorname{minimizar}} & f(\Theta(\mathbf{x})) \\
\text { sujeito a } & g_{k}(\Theta(\mathbf{x})) \leqslant 0 & k=1 . . N_{k} \\
& h_{j}(\Theta(\mathbf{x}))=0 \quad j=1 . . N_{j} \\
& \delta \Pi(\Theta(\mathbf{x}))=0
\end{array}
$$

onde $f(\Theta(\mathbf{x}))$ é a função objetivo (medida de desempenho) do problema de otimização topológica, $g_{k}(\Theta(\mathbf{x})) \leqslant 0$ e $h_{j}(\Theta(\mathbf{x}))=0$ são as restrições de projeto, para $k=1 . . N_{k}$ e $j=1 . . N_{j}$, respectivamente, e $\delta \Pi(\Theta(\mathbf{x}))=0$ indica a condição de equilíbrio da estrutura, representada pela estacionariedade da energia potencial total do sistema, $\Pi(\Theta(\mathbf{x}))$. Idealmente, no problema contínuo de otimização topológica, Equação (2.3), o campo de variáveis de projeto (campo que pode ser modificado para minimizar a função objetivo dentro da região viável de projeto) é representado pela própria função de parametrização discreta $\Theta(\mathbf{x})$; em outras palavras, o campo de variáveis de projeto consiste na própria topologia a ser otimizada.

Segundo Sigmund e Maute (2013), o problema de otimização topológica, Equação (2.3), pode ser abordado de duas formas: 1) com abordagens Lagrangianas, que consistem em métodos de otimização de forma que permitem a inclusão de furos (mudança de topologia) durante o procedimento de otimização; e 2) com abordagens Eulerianas, que consistem em métodos de malha fixa onde o contorno da topologia (fronteira entre as fases sólida e vazia) é dado de forma implícita. Em abordagens Eulerianas, a mudança de topologia é normalmente feita de forma automática e simultânea a mudança de forma da estrutura. Note que ambas as abordagens fazem o uso de métodos numéricos, como o Método dos Elementos Finitos (MEF) baseado em deslocamentos (BATHE, 1996), para a obtenção do estado de equilíbrio da estrutura durante as etapas da otimização. A diferença é que em abordagens Lagrangianas a malha é alinhada ao contorno da topologia, que é dado de forma explícita, enquanto que em abordagens Eulerianas, malhas fixas são utilizadas.

Neste trabalho, a otimização topológica clássica baseada em densidade (SIGMUND; MAUTE, 2013) é empregada. Esta abordagem é baseada na relaxação do espaço de solução por meio da relaxação da parametrização discreta, Equação (2.2). Esta relaxação é empregada com o intuito de permitir uma variação contínua entre as fases sólida e vazia, permitindo o cálculo de derivadas e, consequentemente, a otimização com métodos baseados em gradiente. Nesta abordagem, o domínio fixo de projeto é discretizado com uma malha fixa de elementos finitos, e cada elemento da malha é então associado a uma densidade relativa $\bar{\rho}_{e}$, que varia, de forma contínua, de 0 (fase vazia) até 1 (fase sólida). Grandezas como rigidez, tensão e volume são então calculadas com base na densidade relativa associada a cada elemento da malha (BENDSØE; SIGMUND, 1999). A Figura 5 
Figura 5: Otimização topológica baseada em densidade - ilustração: (a) problema contínuo; (b) problema discretizado; (c) topologia otimizada no domínio discretizado; (d) topologia pós-processada por meio de técnica de extração de contorno.

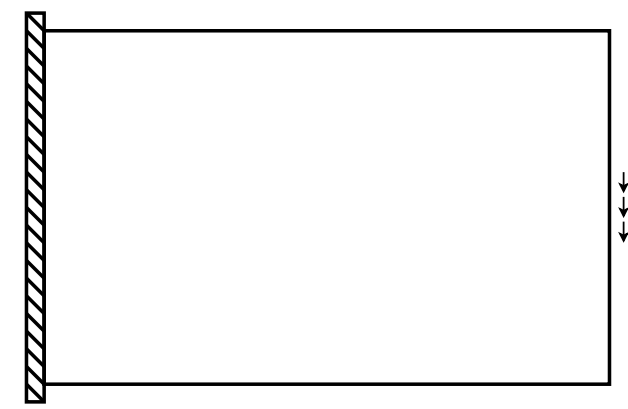

(a)

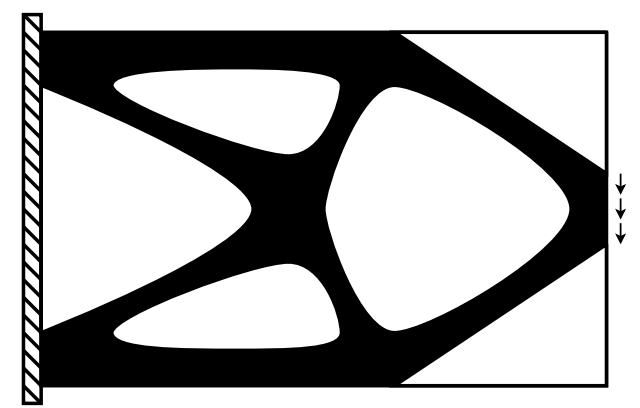

(d)

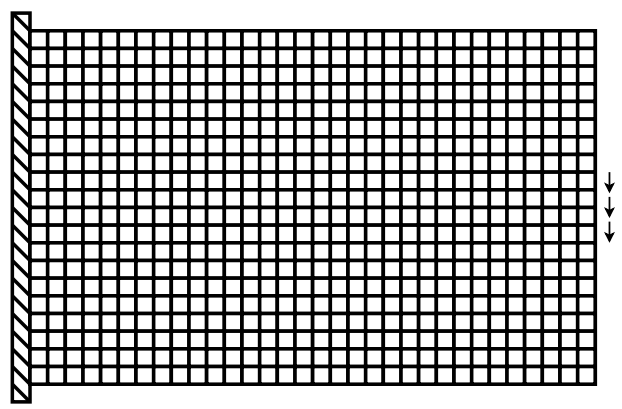

(b)

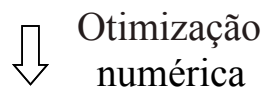

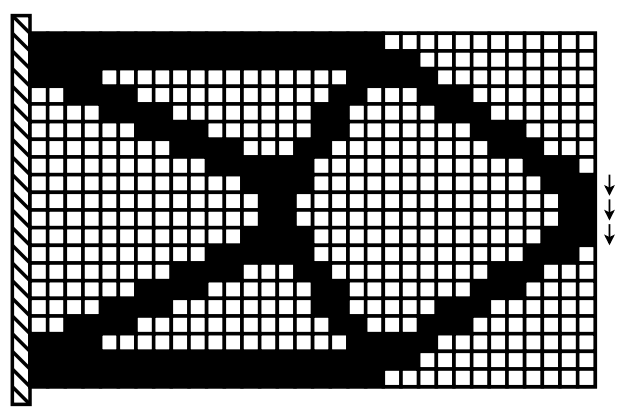

(c)

Fonte: produção do próprio autor.

ilustra esquematicamente a otimização topológica clássica baseada em densidade.

Além da definição do problema de otimização topológica a ser solucionado (minimização de volume sujeito à restrição de tensão, por exemplo), deve-se definir o domínio de projeto, condições de contorno, propriedades e hipóteses de comportamento. O domínio de projeto é então discretizado com uma malha fixa de elementos finitos e, posteriormente, métodos numéricos de otimização são empregados para a obtenção da distribuição de densidades relativas, $\overline{\boldsymbol{\rho}}^{*}$, que minimize a função objetivo ao mesmo tempo em que as restrições de projeto sejam satisfeitas (BENDSØE; SIGMUND, 2003).

A utilização da parametrização contínua permite a obtenção de densidades intermediárias na solução do problema de otimização topológica (BENDSØE; SIGMUND, 2003). Entretanto, buscam-se topologias sem (ou com pouca) presença de densidades intermediárias, de forma que a topologia otimizada represente de fato uma distribuição de material isotrópico. Para tanto, utilizam-se penalização e métodos de projeção (SIGMUND, 2007), conforme apresentado nas próximas seções, que mostram excelentes resultados na obtenção de topologias quase preto e branco (sólido e vazio), inclusive em problemas com restrição de tensão. 
A abordagem baseada em densidade é classificada como uma abordagem de otimização topológica Euleriana (SIGMUND; MAUTE, 2013), pois é uma abordagem de malha fixa onde o contorno da topologia é dado de forma implícita. Desta forma, após a obtenção da topologia otimizada no domínio discretizado, métodos de extração de contorno são empregados para a obtenção explícita do contorno da topologia (SIGMUND; MAUTE, 2013), Figura 5.

Como exemplo de problema de otimização topológica, escreve-se o problema de minimização de flexibilidade com restrição de volume, que consiste no problema clássico de otimização topológica de distribuição de material isotrópico (BENDSØE; SIGMUND, 2003). Considerando a abordagem baseada em densidade e uma discretização do domínio de projeto por meio do MEF baseado em deslocamentos, escreve-se este problema como

$$
\begin{array}{cl}
\underset{\boldsymbol{\rho}}{\operatorname{minimizar}} & f \operatorname{lex}(\overline{\boldsymbol{\rho}})=\mathbf{F}^{T} \mathbf{U}(\overline{\boldsymbol{\rho}}) \\
\text { sujeito a } & V(\overline{\boldsymbol{\rho}}) \leqslant V_{\text {lim }} \\
& 0 \leqslant \rho_{e} \leqslant 1 \\
& \mathbf{K}(\overline{\boldsymbol{\rho}}) \mathbf{U}(\overline{\boldsymbol{\rho}})=\mathbf{F}
\end{array}
$$

onde flex $(\overline{\boldsymbol{\rho}})$ é a flexibilidade da estrutura (compliance), que consiste no produto escalar do vetor de forças externas $\mathbf{F}$ e do vetor de deslocamentos nodais $\mathbf{U}(\overline{\boldsymbol{\rho}}) ; \overline{\boldsymbol{\rho}} \in \mathbb{R}^{N_{e}}$ é o vetor de densidades relativas, que representa a topologia da estrutura no domínio discretizado; $N_{e}$ é o número de elementos da malha; $V(\overline{\boldsymbol{\rho}}) \leqslant V_{\text {lim }}$ é a restrição de volume, que depende do volume da estrutura $V(\overline{\boldsymbol{\rho}})=\sum_{e=1}^{N_{e}} V_{e} \bar{\rho}_{e}$, calculado como o somatório do volume da cada elemento $V_{e}$ ponderado por sua densidade relativa $\bar{\rho}_{e}$, e do volume limite da estrutura $V_{\text {lim }} ; 0 \leqslant \rho_{e} \leqslant 1$ representa as restrições laterais sobre a e-ésima variável de projeto; e $\mathbf{K}(\overline{\boldsymbol{\rho}})$ é a matriz de rigidez global do sistema. Neste caso, hipótese de elasticidade linear foi considerada, de forma que o estado de equilíbrio da estrutura pode ser obtido por meio da solução do sistema de equações lineares representado por $\mathbf{K}(\overline{\boldsymbol{\rho}}) \mathbf{U}(\overline{\boldsymbol{\rho}})=\mathbf{F}$.

A matriz de rigidez global do sistema é obtida pela montagem, em um nível global, da rigidez local de cada elemento da malha. Pode-se escrever esta montagem, de forma simbólica, como

$$
\mathbf{K}(\overline{\boldsymbol{\rho}}) \leftarrow \biguplus_{e=1}^{N_{e}} \mathbf{k}_{e}\left(\bar{\rho}_{e}\right),
$$

onde $\mathbf{k}_{e}\left(\bar{\rho}_{e}\right)$ é a matriz de rigidez local do e-ésimo elemento da malha, obtida como

$$
\mathbf{k}_{e}\left(\bar{\rho}_{e}\right)=\int_{\Omega_{e}} \mathbf{B}_{e}^{T} \mathbf{C}\left(\bar{\rho}_{e}\right) \mathbf{B}_{e} d \Omega_{e} .
$$

Na Equação (2.6), $\Omega_{e}$ é o domínio do elemento, $\mathbf{B}_{e}$ a matriz de transformação deformaçãodeslocamento e $\mathbf{C}\left(\bar{\rho}_{e}\right)$ o tensor constitutivo.

O tensor constitutivo é calculado com base na parametrização contínua SIMP (Solid Isotropic Material with Penalization) (BENDSØE; SIGMUND, 1999; SIGMUND, 
2007)

$$
\mathbf{C}\left(\bar{\rho}_{e}\right)=\left(\rho_{\min }+\left(1-\rho_{\min }\right) \bar{\rho}_{e}^{p}\right) \mathbf{C}^{b}
$$

onde $\mathbf{C}^{b}$ é o tensor constitutivo do material base, $\rho_{\text {min }}$ é uma constante de valor pequeno, incluída na parametrização para evitar instabilidades numéricas, e $p$ é um parâmetro de penalização, usualmente escolhido igual a 3 (BENDSØE; SIGMUND, 1999), de forma a penalizar as densidades intermediárias e obter soluções próximas do espaço 0/1. Note que a parametrização contínua, Equação (2.7), coincide com a parametrização discreta, Equações (2.1) e (2.2), para $\bar{\rho}_{e}=0$ (vazio), desde que $\rho_{\min }=0$, e $\bar{\rho}_{e}=1$ (sólido). Na prática, valores positivos (mas pequenos) são atribuídos a $\rho_{\min }$, como $\rho_{\min }=1 \times 10^{-9}$, de forma a evitar a singularidade durante a solução do sistema de equações de equilíbrio (SIGMUND, 2007).

As variáveis de projeto, em um problema de otimização baseado em densidade, são usualmente representadas por $\boldsymbol{\rho} \in \mathbb{R}^{N_{e}}$. Quando as variáveis de projeto são as próprias densidades relativas, ou seja, se o problema apresentado na Equação (2.4) for solucionado para $\overline{\boldsymbol{\rho}}=\boldsymbol{\rho}$, sem a utilização de métodos de restrição do espaço de solução, dificuldades como dependência da malha e instabilidade de tabuleiro são observadas (BENDSØE; SIGMUND, 2003). Conforme apresentado posteriormente na subseção 2.2, as densidades relativas $\overline{\boldsymbol{\rho}}$, nesta tese, são calculadas com base na projeção de variáveis de projeto filtradas, o que é suficiente para a superação destas dificuldades.

Nesta tese, o problema de minimização de volume com restrição de tensão é abordado, conforme apresentado posteriormente na seção 4. O problema de minimização de flexibilidade, Equação (2.4), é abordado nas próximas subseções unicamente para demonstrar as dificuldades de dependência da malha e instabilidade de tabuleiro, apresentadas a seguir na subseção 2.1 .

\subsection{Dificuldades associadas}

Problemas de otimização topológica baseada em densidade apresentam duas grandes dificuldades (BENDSØE; SIGMUND, 2003): 1) dependência da malha e 2) instabilidade de tabuleiro. Estas dificuldades foram inicialmente observadas, na literatura, em problemas baseados em flexibilidade (SIGMUND; PETERSSON, 1998); no entanto, conforme observado em Duysinx e Bendsøe (1998), problemas baseados em tensão, como aqueles abordados nesta tese, também são afetados.

Além destas, o problema de otimização topológica com restrição de tensão baseado em densidade apresenta algumas dificuldades adicionais, como o fenômeno de singularidade (DUYSINX; BENDSØE, 1998), a natureza local do critério de falha em tensão (PEREIRA; FANCELLO; BARCELLOS, 2004), e a concentração artificial de tensão nas interfaces irregulares entre a fase sólida e a fase vazia da topologia (SVÄRD, 2015). Estas dificuldades 
adicionais estão relacionadas estritamente ao problema baseado em tensão e são abordadas posteriormente na seção 4.

\subsubsection{Dependência da malha}

O problema contínuo de otimização topológica de distribuição de material isotrópico, Equação (2.3), em geral, não possui mínimo global (SIGMUND; PETERSSON, 1998), pois a inclusão de mais e mais furos na topologia, mantendo o volume estrutural, melhora o desempenho da estrutura (SIGMUND; PETERSSON, 1998). No limite deste processo, obtêm-se variações estruturais na forma de microestruturas que maximizam o desempenho do material base. Estas microestruturas são normalmente não isotrópicas e não podem ser obtidas por meio da distribuição de material isotrópico. Neste caso, é dito que o espaço de soluções admissíveis não está fechado, e que não é possível obter a solução (mínimo global) do problema de otimização topológica considerando uma formulação de distribuição de material isotrópico (BENDSØE; SIGMUND, 2003; KOHN; STRANG, 1986a; KOHN; STRANG, 1986b; KOHN; STRANG, 1986c).

Em um domínio discretizado, como a combinação de distribuições de material é finita, o mínimo global existe (BENDSØE; SIGMUND, 2003); no entanto, o problema de não existência de mínimo global também afeta o problema no domínio discretizado, no sentido em que malhas cada vez mais refinadas fornecem soluções cada vez melhores e com um maior número de furos (SIGMUND, 1994). O fenômeno é ilustrado na Figura 6 (b) e (c) para o problema clássico de flexibilidade, Equação (2.4), considerando o domínio de projeto e condições de contorno ilustrados na Figura 1.

Figura 6: Instabilidade de tabuleiro e dependência da malha: (a) exemplo de instabilidade de tabuleiro; (b) solução para discretização com 600 elementos; (c) solução para discretização com 5400 elementos.

(a)

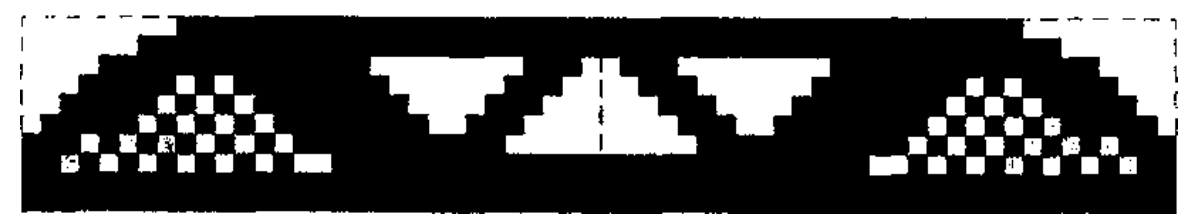

(b)

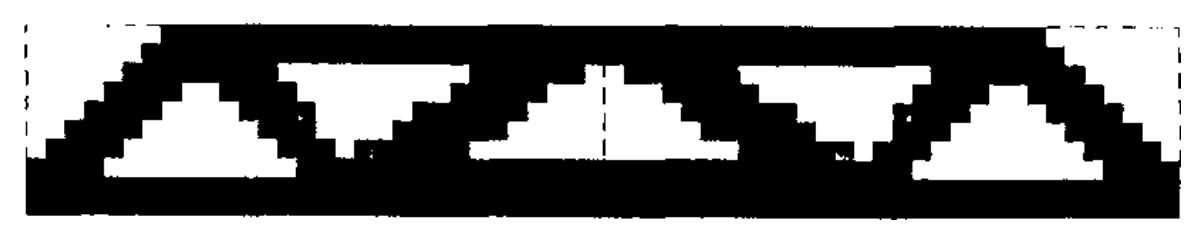

(c)

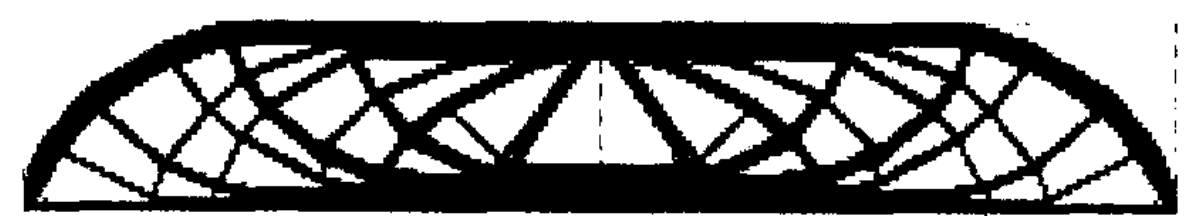

Fonte: adaptado de Sigmund e Petersson (1998). 
A não existência de mínimo global, quando associada ao problema discretizado, é dita dependência da malha, pois malhas diferentes fornecem soluções diferentes. Idealmente, uma solução em uma malha mais refinada deveria ter a mesma topologia de uma solução em uma malha mais grosseira, com diferenças de forma e definição de contorno somente (SIGMUND, 1994).

O problema de não existência de mínimo global e, consequentemente, o problema de dependência da malha, pode ser evitado de duas maneiras (SIGMUND; PETERSSON, 1998): 1) utilizando-se métodos de relaxação do espaço de solução (CHENG; OLHOFF, 1981; KOHN; STRANG, 1986a; KOHN; STRANG, 1986b; KOHN; STRANG, 1986c), como a abordagem de homogeneização para otimização topológica, proposta por Bendsøe e Kikuchi (1988); 2) utilizando-se métodos de restrição do espaço de solução (AMBROSIO; BUTTAZZO, 1993; SIGMUND, 2007), conforme empregado neste trabalho.

Métodos de relaxação funcionam por meio da expansão do espaço de solução, permitindo a utilização de materiais ortotrópicos ou anisotrópicos na solução do problema de otimização topológica (BENDSØE; SIGMUND, 1999). Neste caso, perde-se o conceito de otimização topológica por meio da distribuição de material isotrópico, pois diferentes classes de material poderiam ser obtidas na topologia otimizada. É importante salientar que a utilização da parametrização contínua SIMP também gera uma relaxação no espaço de solução, ao permitir densidades intermediárias na topologia otimizada. No entanto, a relaxação gerada pela parametrização SIMP não é suficiente para evitar o problema de não existência de mínimo global (BENDSØE; SIGMUND, 1999).

Métodos de restrição, por outro lado, funcionam por meio da redução do espaço de solução (SIGMUND; PETERSSON, 1998). Técnicas de restrição do espaço de solução normalmente modificam o problema original, seja por meio da inclusão de restrições adicionais, ou por meio da aplicação de operadores de convolução sobre as grandezas do problema de otimização (SIGMUND; PETERSSON, 1998). Métodos de restrição do espaço de solução são bastante populares, na literatura, principalmente por permitir a solução do problema de otimização topológica por meio da distribuição de material isotrópico somente, sem a necessidade de permitir a utilização de outras classes de material para a correta solução do problema (BENDSØE; SIGMUND, 1999).

Neste trabalho, métodos de restrição do espaço de solução são utilizados para evitar o problema de dependência da malha, conforme apresentado na subseção 2.2 .

\subsubsection{Instabilidade de tabuleiro}

A instabilidade de tabuleiro consiste em um padrão em forma de tabuleiro de xadrez, alternando elementos sólidos e vazios, que surge durante o procedimento de otimização topológica, Figura 6 (a). A instabilidade de tabuleiro é de origem numérica, e surge devido a escolha inadequada das funções de interpolação dos campos de densidades relativas e de 
deslocamentos (JOG; HABER, 1996). Díaz e Sigmund (1995) mostram que, devido a um modelamento ruim associado ao MEF baseado em deslocamentos, a rigidez do padrão em forma de tabuleiro é superestimada em alguns casos, o que leva a preferência do algoritmo de otimização na sua obtenção. Topologias que apresentam padrões em forma de tabuleiro não são consideradas mínimos locais, pois estes padrões não possuem significado físico e, além disso, a sua elevada rigidez é artificial (DÍAZ; SIGMUND, 1995; JOG; HABER, 1996).

A instabilidade de tabuleiro está normalmente associada à utilização de elementos de baixa ordem, como o elemento bilinear isoparamétrico de quatro nós; no entanto, mostra-se, em Díaz e Sigmund (1995), que a utilização de elementos de mais alta ordem, como o elemento biquadrático isoparamétrico de nove nós, não alivia totalmente esta dificuldade em alguns casos.

A instabilidade de tabuleiro pode ser evitada com a utilização de métodos de restrição do espaço de solução (BENDSØE; SIGMUND, 2003), apresentados a seguir na subseção 2.2 .

\subsection{Métodos de restrição do espaço de solução}

Métodos de restrição do espaço de solução são frequentemente empregados na otimização topológica baseada em densidade. Estes métodos, quando devidamente utilizados, evitam problemas de dependência da malha e de instabilidade de tabuleiro (BENDSØE; SIGMUND, 2003). Sigmund (2007) classifica os métodos de restrição do espaço de solução em três categorias: 1) métodos de filtragem independentes da malha, como os filtros de gradiente (SIGMUND, 1994) ou densidade (BRUNS; TORTORELLI, 2001); 2) métodos de restrição, como o método de controle de perímetro (AMBROSIO; BUTTAZZO, 1993); e 3) outros métodos, como as abordagens baseadas em level-set (ALLAIRE; JOUVE; TOADER, 2004; WANG; WANG; GUO, 2003). Existem também os métodos híbridos entre as categorias 1 e 2, como o método proposto por Cardoso e Fonseca (2003), onde os limites móveis do algoritmo de otimização são modificados com método de filtragem (SIGMUND, 2007).

Os métodos de filtragem independentes da malha (categoria 1) são os mais populares entre os métodos de restrição do espaço de solução, devido a sua eficiência (não agregam custo computacional considerável à solução do problema de otimização) e facilidade de implementação, quando comparado às outras abordagens (SIGMUND, 2007).

Neste trabalho, utiliza-se o filtro de densidade com passo adicional de projeção (GUEST; PRÉVOST; BELYTSCHKO, 2004; WANG; LAZAROV; SIGMUND, 2011), que consiste em um método de filtragem independente da malha, conforme apresentado nas subseções 2.2.1 e 2.2.2. 


\subsubsection{Filtro de densidade}

O filtro de densidade tradicional, de Bruns e Tortorelli (2001), foi introduzido na literatura como uma alternativa matematicamente consistente ao filtro de gradiente proposto por Sigmund (1994). Apesar de ter sido originalmente utilizado na solução de problemas de flexibilidade e de mecanismos flexíveis, o filtro de densidade tem sido utilizado na solução de diversos problemas, na literatura, inclusive na solução de problemas baseados em tensão (BRUGGI; DUYSINX, 2012; HOLMBERG; TORSTENFELT; KLARBRING, 2013; KIYONO et al., 2016; LE et al., 2010; LEON et al., 2015; PEREIRA; CARDOSO, 2018; SVÄRD, 2015; WANG; QIAN, 2018). Além disso, o filtro de densidade é utilizado como base para métodos mais sofisticados, como os métodos de projeção descritos na subseção 2.2.2.

Neste trabalho, o filtro de densidade é empregado conforme apresentado em Sigmund (2007): a densidade filtrada $\tilde{\rho}_{e}$ de cada elemento $e$ é obtida por meio da aplicação de uma projeção linear sobre as variáveis de projeto $\boldsymbol{\rho}$, em uma vizinhança circular $\vartheta_{e}$ centrada no elemento $e$, de raio $R$ especificado pelo projetista, como

$$
\tilde{\rho}_{e}=\frac{\sum_{i \in \vartheta_{e}} w\left(\mathbf{x}_{i}\right) V_{i} \rho_{i}}{\sum_{i \in \vartheta_{e}} w\left(\mathbf{x}_{i}\right) V_{i}} .
$$

A função de ponderação linear é definida como

$$
w\left(\mathbf{x}_{i}\right)=\max \left(R-\left\|\mathbf{x}_{i}-\mathbf{x}_{e}\right\|, 0.0\right)
$$

onde $\mathbf{x}_{i}$ são as coordenadas do centro do elemento $i$ e $\mathbf{x}_{e}$ são as coordenadas do centro da vizinhança $\vartheta_{e}$. Conforme ilustrado na Figura 7 , o filtro de densidade pode ser utilizado para eliminar o problema de instabilidade de tabuleiro e aliviar a dependência da malha.

Conforme discutido em Sigmund (2007), a utilização do filtro de densidade fornece resultados que apresentam grandes quantidades de material intermediário entre a fase sólida e a fase vazia da topologia otimizada, mesmo quando elevados valores de $p$ são utilizados, Equação (2.7). Em alguns casos, como na solução do problema tradicional de flexibilidade, transições com grandes quantidades de material intermediário não são um problema, de forma que um simples pós-processamento baseado em curvas de nível leva a estruturas com um contorno suave e desempenho similar a estrutura no domínio discretizado (SIGMUND, 2007). Em outros casos, como no problema baseado em tensão, conforme mostrado e discutido em Silva, Beck e Sigmund (2019), o controle sobre o tamanho da transição de material intermediário, entre as fases sólida e vazia da topologia, é essencial para se obter um resultado com boa acurácia.

Métodos de projeção, conforme apresentados na sequência, são aplicados sobre as densidades filtradas para, dentre outros motivos, reduzir a quantidade de material intermediário na transição entre a fase sólida e a fase vazia da topologia otimizada (SIGMUND, 2007). 
Figura 7: Topologias otimizadas considerando filtro de densidades: (a) 300; (b) 1200; (c) 4800; (d) 19200 elementos.

(a)

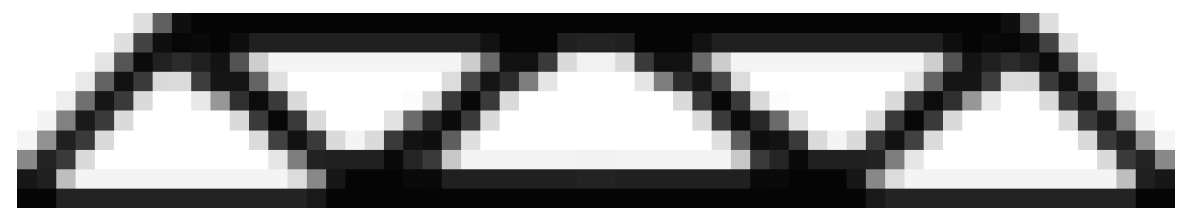

(b)

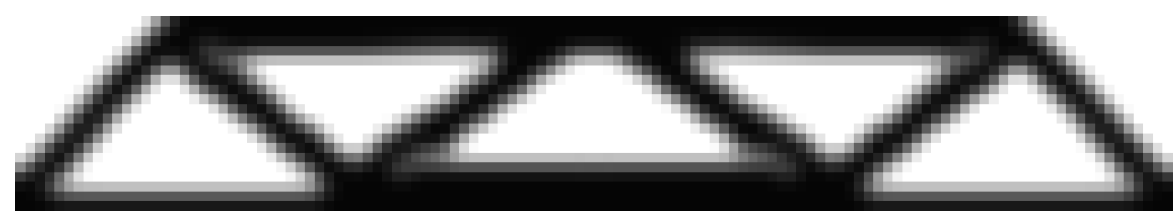

(c)

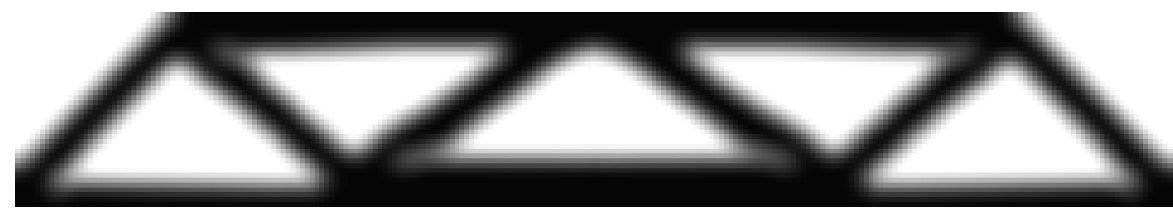

(d)

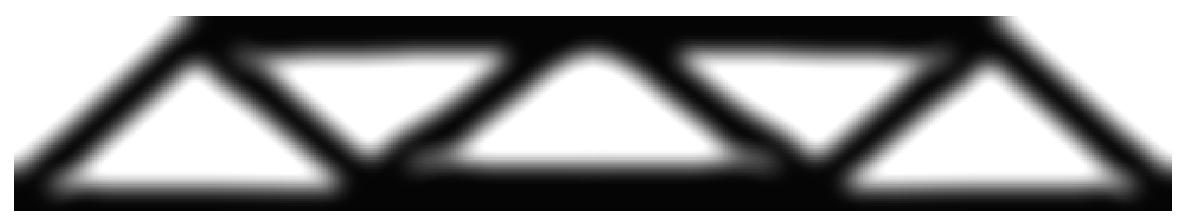

Fonte: Silva (2016).

\subsubsection{Métodos de projeção}

Métodos de projeção consistem em aproximações suaves para a função Heaviside que têm como principal objetivo projetar as densidades filtradas para um espaço 0/1 (WANG; LAZAROV; SIGMUND, 2011). Além disso, estes métodos podem ser utilizados para impor uma escala de comprimento mínimo na solução otimizada (SIGMUND, 2007).

Neste trabalho, dois métodos de projeção são utilizados: 1) o método de projeção de dilatação, proposto por Guest, Prévost e Belytschko (2004); e 2) o método de projeção parametrizada, proposto por Wang, Lazarov e Sigmund (2011).

\subsubsection{Projeção de dilatação}

A projeção de dilatação, proposta por Guest, Prévost e Belytschko (2004), consiste no cálculo das densidades relativas por meio da projeção de cada densidade filtrada $\tilde{\rho}_{e}$ para 1 (fase sólida), caso $\tilde{\rho}_{e}>0$ (SIGMUND, 2007). Com o intuito de utilizar minimizadores baseados em gradiente, Guest, Prévost e Belytschko (2004) propõem o uso de uma aproximação suave para a função Heaviside, tal que

$$
\bar{\rho}_{e}=1-e^{-\delta \tilde{\rho}_{e}}+\tilde{\rho}_{e} e^{-\delta}
$$

onde $\delta$ controla a não linearidade da projeção: para $\delta \rightarrow 0$ uma relação linear entre densidades filtradas e densidades relativas é obtida, enquanto que para $\delta \rightarrow \infty$ uma relação 
que aproxima a função Heaviside é obtida. Guest, Prévost e Belytschko (2004) propõem uma abordagem de continuação para solucionar o problema de otimização topológica: inicialmente o problema é solucionado para $\delta=0$; obtida a solução do problema, aumentase o valor de $\delta$ e soluciona-se o problema novamente; este procedimento é empregado até a obtenção de uma estrutura próxima do espaço $0 / 1$, ou até que o valor máximo $\delta_{\max }$, especificado pelo projetista, seja alcançado. Guest, Prévost e Belytschko (2004) sugerem a abordagem de continuação pela dificuldade de se iniciar o procedimento de otimização com elevados valores de $\delta$, dificuldade esta que se origina na alta não linearidade da função de projeção. Além disso, conforme discutido em Sigmund (2007), a projeção de dilatação não preserva o volume da estrutura filtrada, o que exige a utilização de uma abordagem de continuação consciente, que não apresente incrementos abruptos no parâmetro $\delta$. Ainda segundo Sigmund (2007), a abordagem de continuação permite uma convergência mais estável do procedimento de otimização.

Guest, Prévost e Belytschko (2004) mostram, por meio da solução numérica de problemas de flexibilidade, que a utilização da projeção de dilatação, Equação (2.10), fornece, simultaneamente, topologias próximas do espaço 0/1 e uma escala de comprimento mínimo sobre a fase sólida da topologia otimizada. No entanto, conforme demonstrado por Sigmund (2007) e Wang, Lazarov e Sigmund (2011), a projeção de dilatação não impõe uma escala de comprimento mínimo em todos os casos. O caso patológico de membros conectados por um único nó, por exemplo, que usualmente surge na solução de problemas de mecanismos flexíveis, não é evitado com a utilização da projeção de dilatação; pelo contrário, conforme mostrado nos resultados apresentados em Sigmund (2007) e Wang, Lazarov e Sigmund (2011), a patologia de membros conectados por um único nó fica ainda mais evidente quando a projeção é utilizada. Wang, Lazarov e Sigmund (2011) demonstram, ainda, que a imposição de uma escala de comprimento mínimo não pode ser garantida com a utilização de métodos de projeção tradicionais de uma única etapa, sejam eles de dilatação, erosão ou que preserve o volume da topologia filtrada.

Neste trabalho, o método de projeção de dilatação é utilizado nas três formulações baseadas em tensão sob incerteza nas forças. Este método é utilizado apenas com o intuito de reduzir a transição de material intermediário entre as fases sólida e vazia, que surgem devido a utilização do filtro de densidade. Nenhum estudo foi feito para verificar se as topologias obtidas respeitam uma escala de comprimento mínimo.

\subsubsection{Projeção parametrizada}

A projeção parametrizada, proposta por Xu, Cai e Cheng (2010), consiste no cálculo das densidades relativas por meio da projeção de cada densidade filtrada $\tilde{\rho}_{e}$ para 1 (fase sólida), caso $\tilde{\rho}_{e}>\eta$, e para 0 (fase vazia), caso $\tilde{\rho}_{e}<\eta$, com $\eta \in[0,1]$. Note que, se $\eta=0$, recuperamos a projeção de dilatação (XU; CAI; CHENG, 2010). 
Neste trabalho, emprega-se a aproximação suave para a função Heaviside proposta por Wang, Lazarov e Sigmund (2011), que consiste em uma alternativa à função condicional proposta por Xu, Cai e Cheng (2010). As densidades relativas e filtradas são relacionadas como

$$
\bar{\rho}_{e}=\frac{\tanh (\delta \eta)+\tanh \left(\delta\left(\tilde{\rho}_{e}-\eta\right)\right)}{\tanh (\delta \eta)+\tanh (\delta(1-\eta))} .
$$

O parâmetro $\delta$ controla a não linearidade da projeção parametrizada: $\delta \rightarrow 0$ resulta em uma relação linear entre densidades relativas e filtradas; $\delta \rightarrow \infty$ resulta em uma relação extremamente não linear, que aproxima a função Heaviside. A diferença entre a projeção parametrizada e a projeção de dilatação encontra-se no parâmetro $\eta$, que é inexistente na projeção de dilatação. Este parâmetro controla a quantidade de densidades filtradas que serão projetadas para a fase sólida ou para a fase vazia. Quanto mais próximo de 0 for o valor de $\eta$, maior vai ser a quantidade de densidades filtradas que serão projetadas para 1 e mais próxima a projeção parametrizada estará de uma projeção de dilatação. Por outro lado, quanto mais próximo de 1 for o valor de $\eta$, maior vai ser a quantidade de densidades filtradas que serão projetadas para 0 e mais próxima a projeção parametrizada estará de uma projeção de erosão. Para valores de $\eta \cong 0.5$ não são observadas características de dilatação ou de erosão; neste caso a projeção preserva, de forma aproximada, o volume da topologia filtrada (XU; CAI; CHENG, 2010).

A projeção parametrizada foi proposta por Xu, Cai e Cheng (2010) como uma alternativa mais estável às projeções de dilatação (GUEST; PRÉVOST; BELYTSCHKO, 2004) e de erosão (SIGMUND, 2007). Xu, Cai e Cheng (2010) demonstram que a escolha de $\eta \cong 0.5$ fornece projeções que preservam o volume da topologia filtrada, resultando em uma convergência mais estável quando a abordagem de continuação é utilizada. Além disso, estes mesmos autores demonstram que a escolha de $\eta \cong 0.5$ garante uma topologia próxima do espaço $0 / 1$ para menores valores de $\delta$, quando comparado a projeções de dilatação ou de erosão, por exemplo.

Conforme discutido anteriormente, métodos de projeção, quando aplicados em uma etapa única sobre as densidades filtradas do problema, não garantem uma escala de comprimento mínimo, seja sobre a fase sólida ou vazia da topologia, independentemente do valor de $\eta$ considerado (WANG; LAZAROV; SIGMUND, 2011). Conforme demonstrado em Wang, Lazarov e Sigmund (2011), no entanto, a consideração simultânea de três campos de densidades relativas no problema de otimização topológica, obtidas a partir das densidades filtradas para três valores diferentes de $\eta$, garante uma escala de comprimento mínimo tanto sobre a fase sólida quanto sobre a fase vazia da topologia otimizada.

Neste trabalho, o método de projeção parametrizada é utilizado na formulação baseada em tensão sob incerteza de manufatura. A formulação proposta é baseada na abordagem robusta desenvolvida por Wang, Lazarov e Sigmund (2011) e também faz o uso de três campos de densidades relativas. 


\section{OTIMIZAÇÃO SOB INCERTEZAS}

Problemas de otimização estrutural são frequentemente formulados, na literatura, assumindo um cenário determinístico, onde todos os parâmetros que afetam a medida de desempenho e/ou as restrições de projeto são completamente conhecidos e de valor determinado (GUO et al., 2009).

Quando os requisitos de projeto em um problema de otimização determinístico são dados por restrições de desigualdade, escreve-se este problema como (ARORA, 2012)

$$
\begin{array}{ll}
\underset{\boldsymbol{\rho}}{\operatorname{minimizar}} & f(\boldsymbol{\rho}) \\
\text { sujeito a } & g_{k}(\boldsymbol{\rho}) \leqslant 0 \quad k=1 . . N_{k}
\end{array}
$$

A formulação determinística de um problema de otimização estrutural deve ser utilizada quando todos os parâmetros que afetam a função objetivo, $f(\boldsymbol{\rho})$, e as restrições de projeto, $g_{k}(\boldsymbol{\rho}) \leqslant 0$, são completamente conhecidos e de valor determinado, ou seja, não apresentam incertezas. Entretanto, sabe-se que problemas reais de engenharia estão sempre sujeitos a incertezas (ELISHAKOFF; OHSAKI, 2010; GUO et al., 2009), por exemplo: incertezas nas propriedades do material, cargas aplicadas (intensidade, inclinação, posição), geometria, etc.

Quando a abordagem determinística é empregada na formulação de um problema que apresenta incertezas (negligenciando, desta forma, a influência destas incertezas sobre a resposta do problema de otimização), soluções não ótimas, ou até mesmo inviáveis, podem ser obtidas (MELCHERS; BECK, 2018). A formulação determinística normalmente gera resultados que são não confiáveis e que são muito sensíveis a perturbações nos parâmetros do modelo (GUO et al., 2009), de forma que pequenas variações nestes parâmetros podem levar a falha da estrutura otimizada, conforme demonstrado em Silva, Cardoso e Beck (2018), onde uma pequena variação na inclinação da carga aplicada foi suficiente para ocasionar a falha da estrutura obtida com a formulação determinística.

De forma a obter uma solução otimizada que seja confiável e robusta em relação às incertezas que afetam o problema estrutural, deve-se empregar formulações que levam em conta os efeitos destas incertezas (BECK; GOMES, 2012; BECK et al., 2015; GUO et al., 2009; MIGUEL et al., 2016).

Abordagens de otimização sob incertezas são normalmente divididas em duas categorias (GUO et al., 2009):

1. Abordagens probabilísticas, onde as incertezas são representadas por variáveis e/ou campos aleatórios e propagadas por meio de técnicas probabilísticas de propagação 
de incertezas. Neste caso, trabalha-se com grandezas como valores esperados, desvios padrão e probabilidades de ocorrência.

2. Abordagens não probabilísticas, onde as incertezas são representadas por variáveis incertas-mas-limitadas. Estas abordagens sempre consideram o pior cenário possível para as restrições de projeto, dados os limites de cada variável incerta. Neste caso, informação probabilística das variáveis incertas, como distribuições de probabilidade, não é necessária.

Esta seção visa descrever três abordagens amplamente utilizadas, na literatura, para otimização sob incertezas, sendo duas delas probabilísticas e uma não probabilística. A subseção 3.1 apresenta a abordagem robusta probabilística, que faz o uso de grandezas como valor esperado e desvio padrão. A subseção 3.2 apresenta a abordagem baseada em confiabilidade (probabilística), que trabalha diretamente com probabilidades de ocorrência. A subseção 3.3 apresenta a abordagem robusta não probabilística, que considera o pior cenário possível para as restrições de projeto. Neste trabalho, todas as formulações são apresentadas considerando incertezas que afetam somente as restrições de projeto, ou seja, a função objetivo é considerada determinística. Além disso, considera-se que as variáveis incertas não possuem influência explícita sobre as variáveis de projeto $\boldsymbol{\rho}$, ou seja, estas variáveis são determinísticas.

\subsection{Otimização robusta probabilística}

A abordagem de otimização robusta utilizada neste trabalho é formulada assumindo que todas as incertezas que afetam o problema de otimização (propriedades do material, forças aplicadas, geometria, etc.) estão contidas no vetor $\mathbf{Z} \in \mathbb{R}^{N}$, sendo $N$ o número total de variáveis aleatórias do problema. Cada componente $i$ do vetor $\mathbf{Z}$ consiste em uma variável aleatória $Z_{i}$, de valor esperado $\mathrm{E}\left[Z_{i}\right]$ e desvio padrão $\operatorname{Std}\left[Z_{i}\right]$.

Quando estas incertezas afetam as restrições de projeto, deve-se reescrever o problema de otimização, Equação (3.1), de forma a levar em conta os efeitos destas incertezas. A formulação robusta de um problema de otimização é dada por (PARK et al., 2006)

$$
\begin{array}{ll}
\underset{\boldsymbol{\rho}}{\operatorname{minimizar}} & f(\boldsymbol{\rho}) \\
\text { sujeito a } & \hat{g}_{k}(\boldsymbol{\rho}, \mathbf{Z}) \leqslant 0 \quad k=1 . . N_{k}
\end{array}
$$

onde $\hat{g}_{k}(\boldsymbol{\rho}, \mathbf{Z}) \leqslant 0$ é a $k$-ésima restrição do problema de otimização, com $\hat{g}_{k}(\boldsymbol{\rho}, \mathbf{Z})=$ $\mathrm{E}\left[g_{k}(\boldsymbol{\rho}, \mathbf{Z})\right]+\alpha \operatorname{Std}\left[g_{k}(\boldsymbol{\rho}, \mathbf{Z})\right]$. O parâmetro $\alpha$ é escolhido pelo projetista; este parâmetro controla o número de desvios padrão na restrição de projeto, de forma a aumentar ou reduzir a robustez da solução otimizada. Quanto maior o valor de $\alpha$, maior a diferença entre $\mathrm{E}\left[g_{k}\left(\boldsymbol{\rho}^{*}, \mathbf{Z}\right)\right]$ e $\hat{g}_{k}\left(\boldsymbol{\rho}^{*}, \mathbf{Z}\right)$ na solução otimizada $\left(\operatorname{com} \hat{g}_{k}\left(\boldsymbol{\rho}^{*}, \mathbf{Z}\right)>\mathrm{E}\left[g_{k}\left(\boldsymbol{\rho}^{*}, \mathbf{Z}\right)\right]\right)$ e, 
consequentemente, menor a sensibilidade desta solução a falhas, ou seja, maior a sua robustez.

O parâmetro $\alpha$ não deve ser confundido com o índice de confiabilidade alvo, $\beta_{T}$, usualmente empregado em formulações baseadas em confiabilidade. Diferentemente do parâmetro $\beta_{T}$, o parâmetro $\alpha$, em geral, não é associado de forma direta com a probabilidade de falha admissível do problema. Uma vez que as distribuições de probabilidade das restrições de projeto não são conhecidas no ponto ótimo, não se pode afirmar qual o valor de $\alpha$ necessário para garantir uma probabilidade de falha admissível definida a priori. Deve-se salientar, no entanto, que no caso específico em que as equações de estado limite são lineares, e as distribuições de probabilidade das restrições de projeto são Gaussianas no ponto ótimo, as soluções obtidas com ambas as abordagens são equivalentes, para $\alpha=\beta_{T}$.

No entanto, apesar do parâmetro $\alpha$ não fornecer um controle explícito sobre a probabilidade de falha associada a cada restrição de projeto, pode-se utilizar a desigualdade unilateral de Chebyshev (ROSS, 2010) para uma aproximação conservadora desta grandeza (SILVA; CARDOSO, 2017). Dado uma variável aleatória real $V$, de variância finita, escreve-se a desigualdade unilateral de Chebyshev como

$$
\mathrm{P}(V \geqslant \mathrm{E}[V]+\alpha \operatorname{Std}[V]) \leqslant \frac{1}{1+\alpha^{2}},
$$

onde $P(\cdot)$ indica probabilidade. A desigualdade unilateral de Chebyshev mostra que a probabilidade de $V$ ser maior do que o seu valor esperado somado de $\alpha$ desvios padrão é menor do que $\frac{1}{1+\alpha^{2}}$.

A função $g_{k}(\boldsymbol{\rho}, \mathbf{Z})$, por ser uma função de um vetor de variáveis aleatórias $\mathbf{Z}$, também é uma variável aleatória. Como a Equação (3.3) é válida para qualquer variável aleatória real, independentemente de sua distribuição de probabilidade, esta também é válida para $g_{k}(\boldsymbol{\rho}, \mathbf{Z})$. Desta forma, sabendo que a falha da solução otimizada $\boldsymbol{\rho}^{*}$ ocorre para $g_{k}\left(\boldsymbol{\rho}^{*}, \mathbf{Z}\right)$, e considerando que as restrições de projeto sejam satisfeitas no ponto de ótimo, ou seja, $\hat{g}_{k}\left(\boldsymbol{\rho}^{*}, \mathbf{Z}\right) \leqslant 0$, pode-se escrever, baseando-se na Equação (3.3), a seguinte relação

$$
\mathrm{P}\left(g_{k}\left(\boldsymbol{\rho}^{*}, \mathbf{Z}\right) \geqslant \hat{g}_{k}\left(\boldsymbol{\rho}^{*}, \mathbf{Z}\right)\right) \leqslant \frac{1}{1+\alpha^{2}}
$$

A Equação (3.4) pode ser utilizada, na prática, para uma imposição conservadora de uma probabilidade de falha admissível a $k$-ésima restrição de projeto, por meio da escolha do parâmetro $\alpha$ (SILVA; CARDOSO, 2017). Obviamente, quando a função $g_{k}\left(\boldsymbol{\rho}^{*}, \mathbf{Z}\right)$ apresenta distribuição Gaussiana (ou próxima de uma Gaussiana), a Equação (3.4) fornece probabilidade admissível excessivamente conservadora.

Para solucionar o problema de otimização robusta, o valor esperado e o desvio padrão de $g_{k}(\boldsymbol{\rho}, \mathbf{Z})$ devem ser obtidos. O valor esperado e o desvio padrão de funções que dependem de um conjunto de variáveis aleatórias: $\mathrm{E}\left[g_{k}(\boldsymbol{\rho}, \mathbf{Z})\right]$ e $\operatorname{Std}\left[g_{k}(\boldsymbol{\rho}, \mathbf{Z})\right]$, neste caso; 
são avaliados analiticamente como (ELISHAKOFF, 1999)

$$
\mathrm{E}\left[g_{k}(\boldsymbol{\rho}, \mathbf{Z})\right]=\int_{-\infty}^{\infty} \cdots \int_{-\infty}^{\infty} g_{k}(\boldsymbol{\rho}, \mathbf{z}) f_{\mathbf{Z}}(\mathbf{z}) d z_{1} \ldots d z_{N}
$$

onde $f_{\mathbf{Z}}(\mathbf{z})$ é a função conjunta de densidade de probabilidades de $\mathbf{Z}$, e

$$
\operatorname{Std}\left[g_{k}(\boldsymbol{\rho}, \mathbf{Z})\right]=\sqrt{\operatorname{Var}\left[g_{k}(\boldsymbol{\rho}, \mathbf{Z})\right]}
$$

onde $\operatorname{Var}\left[g_{k}(\boldsymbol{\rho}, \mathbf{Z})\right]$ é a variância de $g_{k}(\boldsymbol{\rho}, \mathbf{Z})$, obtida como

$$
\begin{aligned}
\operatorname{Var}\left[g_{k}(\boldsymbol{\rho}, \mathbf{Z})\right] & =\mathrm{E}\left[\left(g_{k}(\boldsymbol{\rho}, \mathbf{Z})-\mathrm{E}\left[g_{k}(\boldsymbol{\rho}, \mathbf{Z})\right]\right)^{2}\right] \\
& =\int_{-\infty}^{\infty} \ldots \int_{-\infty}^{\infty}\left(g_{k}(\boldsymbol{\rho}, \mathbf{z})-\mathrm{E}\left[g_{k}(\boldsymbol{\rho}, \mathbf{Z})\right]\right)^{2} f_{\mathbf{Z}}(\mathbf{z}) d z_{1} \ldots d z_{N} .
\end{aligned}
$$

Note que a avaliação analítica do valor esperado e do desvio padrão de funções que dependem de um vetor de variáveis aleatórias envolve o cálculo de integrais multidimensionais da função conjunta de densidade de probabilidades, $f_{\mathbf{z}}(\mathbf{z})$, no espaço das variáveis incertas. Na prática, estas grandezas dificilmente são avaliadas de forma analítica (LOPEZ; BECK, 2012). As funções $g_{k}(\boldsymbol{\rho}, \mathbf{Z})$ são usualmente parâmetros avaliados de forma numérica, como deslocamentos ou tensões, obtidos como solução de um problema aproximado, por meio da utilização do MEF, por exemplo. Além disso, a função conjunta de densidade de probabilidades, $f_{\mathbf{z}}(\mathbf{z})$, é dificilmente conhecida, o que torna ainda mais difícil a obtenção analítica dos momentos estatísticos.

Neste trabalho, devido à impossibilidade da avaliação analítica dos momentos estatísticos e à natureza do problema de otimização abordado (problema de larga escala, com milhares de variáveis de projeto e milhares de restrições), optou-se pela utilização do método de perturbação (ELISHAKOFF, 1999; STEFANOU, 2009), que consiste em uma abordagem aproximada e de baixo custo computacional, também utilizada por outros autores na otimização estrutural robusta (ASADPOURE; TOOTKABONI; GUEST, 2011; KOGISO et al., 2008; LAZAROV; SCHEVENELS; SIGMUND, 2012). Neste trabalho, utiliza-se o método de perturbação de primeira ordem, que consiste na obtenção aproximada de $g_{k}(\boldsymbol{\rho}, \mathbf{Z})$ por meio de sua expansão em série de Taylor de primeira ordem em torno do valor médio de $\mathbf{Z}$, como

$$
\tilde{g}_{k}(\boldsymbol{\rho}, \mathbf{Z})=g_{k}(\boldsymbol{\rho}, \mathrm{E}[\mathbf{Z}])+\sum_{i=1}^{N}\left(\left.\frac{\partial g_{k}(\boldsymbol{\rho}, \mathbf{z})}{\partial z_{i}}\right|_{\mathbf{z}=\mathrm{E}[\mathbf{Z}]}\right)\left(Z_{i}-\mathrm{E}\left[Z_{i}\right]\right) .
$$

Substitui-se, então, a função aproximada $\tilde{g}_{k}(\boldsymbol{\rho}, \mathbf{Z})$ na Equação (3.5), de forma a obter o seu valor esperado

$$
\mathrm{E}\left[\tilde{g}_{k}(\boldsymbol{\rho}, \mathbf{Z})\right]=g_{k}(\boldsymbol{\rho}, \mathrm{E}[\mathbf{Z}]) .
$$

Posteriormente, substitui-se tanto a função aproximada $\tilde{g}_{k}(\boldsymbol{\rho}, \mathbf{Z})$ quanto o seu valor esperado $\mathrm{E}\left[\tilde{g}_{k}(\boldsymbol{\rho}, \mathbf{Z})\right]$ na Equação (3.7), de forma a obter a sua variância

$$
\operatorname{Var}\left[\tilde{g}_{k}(\boldsymbol{\rho}, \mathbf{Z})\right]=\sum_{i=1}^{N} \sum_{j=1}^{N}\left(\left.\frac{\partial g_{k}(\boldsymbol{\rho}, \mathbf{z})}{\partial z_{i}}\right|_{\mathbf{z}=\mathrm{E}[\mathbf{Z}]}\right)\left(\left.\frac{\partial g_{k}(\boldsymbol{\rho}, \mathbf{z})}{\partial z_{j}}\right|_{\mathbf{z}=\mathrm{E}[\mathbf{Z}]}\right) \operatorname{Cov}\left[Z_{i}, Z_{j}\right],
$$


onde $\operatorname{Cov}\left[Z_{i}, Z_{j}\right]$ representa a covariância entre as variáveis aleatórias $Z_{i}$ e $Z_{j}$. Note que, neste caso, onde a abordagem de perturbação de primeira ordem é empregada, o valor esperado consiste na própria função avaliada no valor médio das variáveis aleatórias. Além disso, verifica-se que as derivadas da função $g_{k}(\boldsymbol{\rho}, \mathbf{Z})$ em relação às variáveis incertas são necessárias para a avaliação da variância. Estas são normalmente obtidas de maneira analítica, conforme mostrado posteriormente na subseção 5.1.1, onde as derivadas são obtidas para o problema baseado em tensão, sendo $g_{k}(\boldsymbol{\rho}, \mathbf{Z})$ a tensão equivalente de von Mises no ponto $k$ e $\mathbf{Z}$ um vetor de ângulos e/ou intensidades (de forças externas) incertos. O desvio padrão é obtido posteriormente com a Equação (3.6), após o cálculo da variância, Equação (3.10).

Apesar de técnicas de simulação, como o método de Simulação de Monte Carlo (SMC), fornecerem resultados com uma maior acurácia (principalmente quando grandes perturbações são consideradas nas variáveis de entrada) (STEFANOU, 2009), estas técnicas são de elevado custo computacional, não sendo apropriadas na propagação de incertezas em problemas de otimização de larga escala, com milhares de variáveis de projeto (ASADPOURE; TOOTKABONI; GUEST, 2011). As técnicas de simulação, no entanto, são algumas vezes utilizadas na validação dos resultados obtidos por meio de técnicas aproximadas (ASADPOURE; TOOTKABONI; GUEST, 2011; SILVA; BECK; CARDOSO, 2018; SILVA; CARDOSO, 2017; TOOTKABONI; ASADPOURE; GUEST, 2012).

\subsection{Otimização baseada em confiabilidade}

O problema de otimização baseado em confiabilidade é escrito como (LOPEZ; BECK, 2012)

$$
\begin{array}{ll}
\underset{\boldsymbol{\rho}}{\operatorname{minimizar}} & f(\boldsymbol{\rho}) \\
\text { sujeito a } & \mathrm{P}\left(g_{k}(\boldsymbol{\rho}, \mathbf{Z})>0\right) \leqslant \mathrm{P}_{a d m} \quad k=1 . . N_{k}
\end{array}
$$

onde $\mathrm{P}_{a d m}$ é a probabilidade de falha admissível, imposta pelo projetista. Esta formulação impõe, como restrição de projeto, que a probabilidade de ocorrência do evento $g_{k}(\boldsymbol{\rho}, \mathbf{Z})>0$, que representa a falha, seja limitada por uma probabilidade de falha admissível.

A avaliação analítica da probabilidade de falha é dada pela integral multidimensional da função conjunta de densidade de probabilidades, $f_{\mathbf{Z}}(\mathbf{z})$, sobre o domínio de falha (LOPEZ; BECK, 2012), tal que

$$
\mathrm{P}\left(g_{k}(\boldsymbol{\rho}, \mathbf{Z})>0\right)=\int_{g_{k}(\boldsymbol{\rho}, \mathbf{Z})>0} f_{\mathbf{Z}}(\mathbf{z}) d \mathbf{z}
$$

A avaliação analítica da probabilidade de falha, no entanto, dificilmente é realizada na prática, pois a função conjunta de densidade de probabilidades, $f_{\mathbf{Z}}(\mathbf{z})$, dificilmente é 
conhecida e o domínio de falha raramente possui expressão analítica (LOPEZ; BECK, 2012). Em aplicações práticas de engenharia, abordagens aproximadas, como os métodos de confiabilidade de primeira ou de segunda ordem (DITLEVSEN; MADSEN, 2007), e abordagens de simulação, como o método de SMC, são utilizadas.

Neste trabalho, devido à natureza de larga escala do problema de otimização abordado, optou-se pela utilização de uma abordagem de primeira ordem. Especificamente, optou-se por formular o problema de otimização baseado em confiabilidade utilizando-se o PMA (Performance Measure Approach) (TU; CHOI; PARK, 1999), que consiste em uma abordagem de primeira ordem amplamente empregada na otimização estrutural baseada em confiabilidade (ALLEN; MAUTE, 2005; CHO et al., 2011; JUNG; CHO, 2004; KANG; LIU, 2018; LUO et al., 2014; MAUTE; FRANGOPOL, 2003; YOUN; CHOI, 2003; YOUN; CHOI; PARK, 2003; YOUN et al., 2004).

Abordagens de primeira ordem são baseadas em um procedimento de duas etapas (LOPEZ; BECK, 2012):

1. Mapeamento de $\mathbb{Z} \rightarrow \mathbb{Y}$, ou seja, do espaço de projeto $\mathbb{Z}$, onde as variáveis aleatórias $\mathbf{Z}$ são definidas, para o espaço normal padrão $\mathbb{Y}$, onde as variáveis aleatórias normal padrão Y são definidas;

2. Linearização da função $g_{k}(\boldsymbol{\rho}, \mathbf{Y})$ no espaço normal padrão.

A probabilidade de falha considerando a função linearizada, representada aqui como $\bar{g}_{k}(\boldsymbol{\rho}, \mathbf{Y})$, pode ser obtida com a expressão analítica, Equação (3.12), pois tanto a função conjunta de densidade de probabilidades, $f_{\mathbf{Y}}(\mathbf{y})=\phi_{n}(\mathbf{y})$, que consiste na distribuição normal padrão multidimensional, quanto o domínio de falha $\bar{g}_{k}(\boldsymbol{\rho}, \mathbf{Y})>0$, com $\bar{g}_{k}(\boldsymbol{\rho}, \mathbf{Y})$ linear, são bem conhecidos. Calcula-se a probabilidade de falha como $\mathrm{P}\left(\bar{g}_{k}(\boldsymbol{\rho}, \mathbf{Y})>0\right)=\Phi\left(-\beta_{k}\right)$, onde $\Phi$ é a função de distribuição cumulativa normal padrão e $\beta_{k}$ é a menor distância entre a função $\bar{g}_{k}(\boldsymbol{\rho}, \mathbf{Y})$ e a origem do espaço normal padrão, também conhecido como índice de confiabilidade de Hasofer-Lind (MELCHERS; BECK, 2018). Neste caso, pode-se reescrever o problema definido na Equação (3.11) como

$$
\begin{array}{ll}
\underset{\boldsymbol{\rho}}{\operatorname{minimizar}} & f(\boldsymbol{\rho}) \\
\text { sujeito a } & \Phi\left(-\beta_{k}\right) \leqslant \Phi\left(-\beta_{T}\right) \quad k=1 . . N_{k}
\end{array},
$$

onde $\Phi\left(-\beta_{T}\right)=\mathrm{P}_{a d m}$, e $\beta_{T}$ é o índice de confiabilidade alvo (parâmetro definido pelo projetista).

A linearização de $g_{k}(\boldsymbol{\rho}, \mathbf{Y})$ é feita, usualmente, sobre o ponto no domínio de falha que apresenta a maior probabilidade de ocorrência, de forma a obter um menor erro entre a probabilidade de falha obtida com a função linearizada e a probabilidade de falha 
exata do problema (LOPEZ; BECK, 2012). No PMA, a linearização de $g_{k}(\boldsymbol{\rho}, \mathbf{Y})$ é feita no ponto $\left(\mathbf{Y}^{(k)}\right)^{*}$, também conhecido como ponto de mínimo desempenho, que coincide com o ponto que apresenta a maior probabilidade de ocorrência quando a restrição está ativa (AOUES; CHATEAUNEUF, 2010). O ponto $\left(\mathbf{Y}^{(k)}\right)^{*}$ é obtido sobre uma esfera $N$-dimensional, definida pelo índice de confiabilidade alvo $\beta_{T}$, por meio de uma análise de confiabilidade inversa no espaço normal padrão, dada por um problema de otimização, tal que (TU; CHOI; PARK, 1999)

$$
\begin{gathered}
\underset{\mathbf{Y}}{\operatorname{minimizar}} \quad-g_{k}(\boldsymbol{\rho}, \mathbf{Y}) \\
\text { sujeito a }
\end{gathered}, \mathbf{Y} \|=\beta_{T},
$$

onde $\|\mathbf{Y}\|$ é a norma euclidiana do vetor $\mathbf{Y}$. No problema definido na Equação (3.14), as variáveis de projeto são as próprias variáveis $\mathbf{Y}$.

Os pontos de mínimo desempenho $\left(\mathbf{Y}^{(k)}\right)^{*}$ fornecem os valores críticos de $g_{k}(\boldsymbol{\rho}, \mathbf{Y})$, que por sua vez são utilizados na solução do problema de otimização baseado em confiabilidade, dado por

$$
\begin{array}{ll}
\underset{\boldsymbol{\rho}}{\operatorname{minimizar}} & f(\boldsymbol{\rho}) \\
\text { sujeito a } & g_{k}\left(\boldsymbol{\rho},\left(\mathbf{Y}^{(k)}\right)^{*}\right) \leqslant 0 \quad k=1 . . N_{k}
\end{array}
$$

onde $g_{k}\left(\boldsymbol{\rho},\left(\mathbf{Y}^{(k)}\right)^{*}\right) \leqslant 0$ é a $k$-ésima restrição de projeto. No problema definido na Equação (3.15), $\boldsymbol{\rho}$ contém as variáveis de projeto.

A abordagem acoplada, que define o PMA, funciona da seguinte forma: a cada passo do problema externo de otimização, Equação (3.15) (associada ao problema estrutural), solucionam-se $N_{k}$ problemas de otimização internos (um para cada restrição de projeto), Equação (3.14) (análises de confiabilidade inversa), de forma a obter $N_{k}$ pontos de mínimo desempenho $\left(\mathbf{Y}^{(k)}\right)^{*}$, que fornecem os valores críticos de $g_{k}(\boldsymbol{\rho}, \mathbf{Y})$, utilizados durante a otimização externa. Obviamente, as soluções obtidas por meio da utilização desta abordagem estão limitadas às aproximações de primeira ordem realizadas, ou seja, uma solução exata, com $\mathrm{P}_{a d m}=\Phi\left(-\beta_{k}\right)$, seria somente obtida para $g_{k}(\boldsymbol{\rho}, \mathbf{Y})$ linear no espaço normal padrão. Na prática, soluções aproximadas são obtidas.

\subsection{Otimização robusta não probabilística}

O problema de otimização robusto não probabilístico é formulado considerando um conjunto de variáveis incertas-mas-limitadas, definido pelo vetor $\mathbf{W} \in \mathbb{R}^{N}$, onde $\mathbf{W} \in[\underline{\mathbf{W}}, \overline{\mathbf{W}}]$. Cada componente $i$ do vetor $\mathbf{W}$, definido como $W_{i}$, representa uma variável incerta no problema de otimização, limitada por um valor mínimo $\underline{W}_{i}$ e por um valor máximo $\bar{W}_{i}$. O objetivo do problema de otimização robusto não probabilístico consiste na 
obtenção de uma solução otimizada que respeite o pior cenário possível para cada restrição de projeto (ELISHAKOFF; OHSAKI, 2010). O problema robusto não probabilístico, tal como o problema de otimização baseado em confiabilidade formulado com o PMA, consiste em uma abordagem acoplada, onde o problema externo de otimização é dado por

$$
\begin{array}{ll}
\underset{\boldsymbol{\rho}}{\operatorname{minimizar}} & f(\boldsymbol{\rho}) \\
\text { sujeito a } & g_{k}\left(\boldsymbol{\rho},\left(\mathbf{W}^{(k)}\right)^{*}\right) \leqslant 0 \quad k=1 . . N_{k},
\end{array}
$$

e os problemas internos são dados por

$$
\begin{array}{ll}
\underset{\mathbf{W}}{\operatorname{maximizar}} & g_{k}(\boldsymbol{\rho}, \mathbf{W}) \\
\text { sujeito a } & \underline{\mathbf{W}} \leqslant \mathbf{W} \leqslant \overline{\mathbf{W}}
\end{array}
$$

onde $\left(\mathbf{W}^{(k)}\right)^{*}$ é o pior cenário associado a $k$-ésima restrição de projeto, obtido por meio da solução do problema definido na Equação (3.17). Os problemas de otimização internos são comumente chamados de problemas de anti-otimização na literatura de otimização estrutural (ELISHAKOFF; HAFTKA; FANG, 1994; GURAV; GOOSEN; VANKEULEN, 2005; LOMBARDI, 1998; LOMBARDI; HAFTKA, 1998), nomenclatura a qual também é adotada nesta tese.

A abordagem acoplada, também conhecida como abordagem de otimização com anti-otimização (ELISHAKOFF; OHSAKI, 2010), funciona de forma similar ao PMA: a cada passo do problema externo de otimização, Equação (3.16), solucionam-se $N_{k}$ problemas de otimização internos, Equação (3.17), de forma a obter $N_{k}$ pontos $\left(\mathbf{W}^{(k)}\right)^{*}$ que fornecem os valores máximos de $g_{k}(\boldsymbol{\rho}, \mathbf{W})$, utilizados durante a otimização externa.

Segundo Elishakoff e Ohsaki (2010), a anti-otimização é uma alternativa e um complemento aos métodos probabilísticos tradicionais. A anti-otimização pode ser utilizada independentemente da distribuição de probabilidade das variáveis incertas $\mathbf{W}$, desde que estas sejam limitadas. Obviamente, conforme discutido em Guo et al. (2009), de forma a garantir a robustez das soluções otimizadas por meio da abordagem não probabilística, as soluções dos problemas internos de anti-otimização, $\left(\mathbf{W}^{(k)}\right)^{*}$, devem ser ótimos globais. Uma resposta obtida que não seja ótimo global, como solução da Equação (3.17), para pelo menos uma restrição de projeto, afeta a robustez da solução do problema de otimização externo. Neste caso, a solução do problema externo fica suscetível a falhas, pois existem realizações de $\mathbf{W}$ que violam pelo menos uma restrição de projeto.

Apesar da abordagem robusta não probabilística ser bastante atrativa, por necessitar somente de limites máximos e mínimos para as variáveis incertas, esta abordagem pode fornecer resultados excessivamente conservadores em alguns casos (GUO et al., 2009). Segundo Ben-Tal e Nemirovski (2002), por exemplo, quando os parâmetros incertos em uma desigualdade linear são de natureza estocástica, é altamente improvável que 
estes apresentem o pior cenário de forma simultânea. No entanto, conforme discutido em Elishakoff e Ohsaki (2010), as abordagens probabilísticas e não probabilística se complementam, sendo de responsabilidade do projetista a escolha entre estas abordagens de forma a melhor solucionar o problema em estudo. 



\section{OTIMIZAÇÃO TOPOLÓGICA COM RESTRIÇÃO DE TENSÃO}

O problema contínuo de otimização topológica com restrição de tensão foi inicialmente abordado em Duysinx e Bendsøe (1998), onde a abordagem baseada em densidade é empregada para a obtenção de estruturas de mínima massa que respeitam um critério de falha em tensão estabelecido a priori. Desde então, o problema baseado em tensão ganhou bastante popularidade na literatura (AMSTUTZ; NOVOTNY, 2010; AMSTUTZ; NOVOTNY; NETO, 2012; BRUGGI, 2008; BRUGGI; DUYSINX, 2012; DUYSINX; SIGMUND, 1998; EMMENDOERFER; FANCELLO, 2014; EMMENDOERFER; FANCELLO, 2016; EMMENDOERFER; SILVA; FANCELLO, 2019; FANCELLO, 2006; FANCELLO; PEREIRA, 2003; HOLMBERG; TORSTENFELT; KLARBRING, 2013; KIYONO; SILVA; REDDY, 2016; KIYONO et al., 2016; LE et al., 2010; LIAN et al., 2017; LEON et al., 2015; LONG; WANG; LIU, 2018; LOPES; NOVOTNY, 2016; LUO; WANG; KANG, 2013; PARÍS et al., 2009; PEREIRA; CARDOSO, 2018; PEREIRA; FANCELLO; BARCELLOS, 2004; PICELLI et al., 2018; SANTOS; LOPES; NOVOTNY, 2017; SVÄRD, 2015; TROYA; TORTORELLI, 2018; WANG; QIAN, 2018), devido principalmente a necessidade de se considerar requisitos de projeto mais realísticos na otimização estrutural.

Considerando o critério de falha de von Mises e hipótese de elasticidade linear, escreve-se o problema de minimização de volume com restrição de tensão como (BRUGGI; DUYSINX, 2012; LE et al., 2010)

$$
\begin{array}{cll}
\underset{\boldsymbol{\rho}}{\operatorname{minimizar}} & V(\overline{\boldsymbol{\rho}}) & \\
\text { sujeito a } & \frac{\sigma_{e q}^{(k)}(\overline{\boldsymbol{\rho}})}{\sigma_{y}}-1 \leqslant 0 & k=1 . . N_{k} \\
& 0 \leqslant \rho_{e} \leqslant 1 & e=1 . . N_{e} \\
& \mathbf{K}(\overline{\boldsymbol{\rho}}) \mathbf{U}(\overline{\boldsymbol{\rho}})=\mathbf{F} &
\end{array}
$$

onde $V(\overline{\boldsymbol{\rho}})$ é o volume da estrutura, $\sigma_{e q}^{(k)}(\overline{\boldsymbol{\rho}})$ é a tensão equivalente de von Mises avaliada no ponto $k, N_{k}$ é o número de pontos onde a tensão de von Mises é avaliada e $\sigma_{y}$ é uma tensão limite, imposta pelo projetista.

Conforme discutido em Sharma e Maute (2018), a formulação considerada nesta tese, Equação (4.1), é mal posta, no sentido em que a solução ótima deste problema consiste em uma estrutura de volume zero. Assumindo que as restrições de tensão em elementos que representam o vazio não sejam levadas em consideração, verifica-se que a solução trivial é a solução óbvia deste problema de otimização. Entretanto, a escolha de uma função de interpolação adequada, que penalize as tensões nas densidades intermediárias, consegue guiar o algoritmo de forma a evitar a solução trivial, conforme discutido em Le et al. (2010), desde que a solução trivial não seja empregada como estimativa inicial no procedimento 
de otimização. Sharma e Maute (2018) propõem a utilização de uma restrição de volume adicional às restrições de tensão, com o intuito de assegurar uma solução otimizada que não seja a solução trivial. Alternativamente, uma restrição de flexibilidade adicional pode ser considerada na formulação (BRUGGI; DUYSINX, 2012). Entretanto, deve-se salientar que as soluções dos problemas modificados (com restrições adicionais) não são iguais a solução do problema original. Na prática, a formulação empregada nesta tese, Equação (4.1), é utilizada sem dificuldades por diversos autores na literatura (BRUGGI, 2008; BRUGGI; DUYSINX, 2012; FANCELLO, 2006; FANCELLO; PEREIRA, 2003; HOLMBERG; TORSTENFELT; KLARBRING, 2013; LE et al., 2010; LUO; WANG; KANG, 2013; PARÍS et al., 2009; PEREIRA; FANCELLO; BARCELLOS, 2004; SVÄRD, 2015; WANG; QIAN, 2018).

A tensão de von Mises é calculada como (DUYSINX; BENDSØE, 1998)

$$
\sigma_{e q}^{(k)}(\overline{\boldsymbol{\rho}})=\sqrt{\overline{\boldsymbol{\sigma}}_{k}^{T}(\overline{\boldsymbol{\rho}}) \mathbf{M} \overline{\boldsymbol{\sigma}}_{k}(\overline{\boldsymbol{\rho}})}
$$

onde $\overline{\boldsymbol{\sigma}}_{k}(\overline{\boldsymbol{\rho}})$ é o vetor tensão, obtido como

$$
\overline{\boldsymbol{\sigma}}_{k}(\overline{\boldsymbol{\rho}})=\mathbf{C}^{b} \mathbf{B}_{k} \mathbf{u}_{k}(\overline{\boldsymbol{\rho}}),
$$

onde $\mathbf{C}^{b}$ é o tensor constitutivo do material base, $\mathbf{B}_{k}$ é a matriz de transformação deformação-deslocamento e $\mathbf{u}_{k}(\overline{\boldsymbol{\rho}})$ é o vetor local de deslocamentos, extraído do vetor global de deslocamentos como $\mathbf{u}_{k}(\overline{\boldsymbol{\rho}})=\mathbf{H}_{k} \mathbf{U}(\overline{\boldsymbol{\rho}})$, onde $\mathbf{H}_{k}$ representa o operador de localização (BATHE, 1996).

Para problemas de estado plano de tensão, define-se a matriz $\mathbf{M}$ como (DUYSINX; BENDSØE, 1998)

$$
\mathbf{M}=\left[\begin{array}{ccc}
1 & -\frac{1}{2} & 0 \\
-\frac{1}{2} & 1 & 0 \\
0 & 0 & 3
\end{array}\right]
$$

A formulação baseada em densidade com critério de falha em tensão, Equação (4.1), está sujeita às dificuldades previamente discutidas na subseção 2.1, de dependência da malha e instabilidade de tabuleiro. Desta forma, a utilização de métodos de restrição do espaço de solução, como o método de filtragem com passo adicional de projeção, empregado neste trabalho, é necessária.

Além destas dificuldades, a formulação baseada em tensão está sujeita a três dificuldades adicionais: o fenômeno de singularidade, subseção 4.1; a natureza local do critério de falha em tensão, subseção 4.2; e a concentração artificial de tensão na interface entra a fase sólida e a fase vazia da topologia, subseção 4.3. As próximas subseções são destinadas a descrição destas dificuldades, tal como a apresentação de possíveis contramedidas que são usualmente empregadas para evitá-las. 


\subsection{Fenômeno de singularidade}

O fenômeno de singularidade consiste em uma irregularidade no espaço de solução devido a descontinuidade da restrição de tensão em $\bar{\rho}=0$ (CHENG; GUO, 1997; DUYSINX; BENDSØE, 1998). Segundo Duysinx e Bendsøe (1998) e Duysinx e Sigmund (1998), quando a densidade relativa associada com um elemento finito tende a zero, as tensões deste elemento tendem a valores finitos (valores algumas vezes maiores do que a tensão limite). Por outro lado, quando a densidade relativa associada com um elemento finito é zero, as tensões neste elemento não deveriam ser levadas em consideração, pois este elemento representa o vazio (sem material); ocasionando, desta forma, uma descontinuidade da restrição de tensão em $\bar{\rho}=0$ (DUYSINX; BENDSØE, 1998).

Devido a descontinuidade das restrições de tensão, a região viável do problema de otimização pode ser muito complexa, contendo subespaços degenerados (CHENG; GUO, 1997). Nestas situações, algoritmos baseados nas condições de Karush-Kuhn-Tucker (baseados em gradiente) se tornam inúteis na obtenção de um ponto de ótimo verdadeiro, obtendo pontos não ótimos em vez disso (CHENG; GUO, 1997).

De forma a superar o fenômeno de singularidade e permitir a solução do problema baseado em tensão com métodos baseados em gradiente, deve-se relaxar as restrições de tensão do problema de otimização (CHENG; GUO, 1997). A ideia essencial de técnicas de relaxação consiste em garantir a continuidade da restrição de tensão em $\bar{\rho}=0$ e, ao mesmo tempo, garantir a sua viabilidade quando $\bar{\rho} \rightarrow 0$ (CHENG; GUO, 1997).

A forma mais simples de relaxar as restrições de tensão consiste na ponderação das tensões $\overline{\boldsymbol{\sigma}}_{k}(\overline{\boldsymbol{\rho}})$, Equação (4.3), com uma função de interpolação de tensão contínua $f_{\sigma}\left(\bar{\rho}_{k}\right)$, como

$$
\boldsymbol{\sigma}_{k}(\overline{\boldsymbol{\rho}})=f_{\sigma}\left(\bar{\rho}_{k}\right) \overline{\boldsymbol{\sigma}}_{k}(\overline{\boldsymbol{\rho}}),
$$

onde a função de interpolação de tensão é escolhida de forma a garantir: 1) $f_{\sigma}(0)=0$, garantindo consequentemente tanto a continuidade da restrição de tensão em $\bar{\rho}_{k}=0$ quanto a sua viabilidade quando $\bar{\rho}_{k} \rightarrow 0$; e 2) $f_{\sigma}(1)=1$, garantindo uma tensão fisicamente consistente na fase sólida da topologia.

Duysinx e Bendsøe (1998) afirmam que o vetor tensão $\overline{\boldsymbol{\sigma}}_{k}(\overline{\boldsymbol{\rho}})$, Equação (4.3), é fisicamente coerente, e que a utilização de qualquer função $f_{\sigma}\left(\bar{\rho}_{k}\right) \neq 1$ na Equação (4.5), para calcular $\boldsymbol{\sigma}_{k}(\overline{\boldsymbol{\rho}})$, traria inconsistências físicas no cálculo das tensões. Entretanto, conforme discutido em Bruggi (2008) e Le et al. (2010), desde que topologias 0/1 sejam obtidas, ou seja, sem (ou com pouca) presença de densidades intermediárias, a escolha apropriada de uma função $f_{\sigma}\left(\bar{\rho}_{k}\right)$ pode ser feita para auxiliar a solução do problema com otimizadores baseados em gradiente.

Le et al. (2010) sugerem que a escolha de $f_{\sigma}\left(\bar{\rho}_{k}\right)$ é flexível e deve ser feita de forma a penalizar as densidades intermediárias e obter topologias 0/1. Exemplos de funções de 
interpolação de tensão obtidas na literatura:

- $f_{\sigma}^{\varepsilon}\left(\bar{\rho}_{k}\right)=\frac{\bar{\rho}_{k}}{\varepsilon\left(1-\bar{\rho}_{k}\right)+\bar{\rho}_{k}}$ (DUYSINX; SIGMUND, 1998), onde $\varepsilon$ é uma constante escolhida pelo projetista, usualmente $0<\varepsilon \leqslant 1$;

- $f_{\sigma}^{q p}\left(\bar{\rho}_{k}\right)=\bar{\rho}_{k}^{p-q}$ (BRUGGI, 2008), onde a constante $p$ vem da parametrização SIMP e a constante $q$ é escolhida pelo projetista, usualmente $2 \leqslant q<3$;

- $f_{\sigma}^{\delta}\left(\bar{\rho}_{k}\right)=1-e^{-\delta_{\sigma} \bar{\rho}_{k}}+\bar{\rho}_{k} e^{-\delta_{\sigma}}$ (SILVA; BECK, 2018), onde $\delta_{\sigma} \geqslant 0$ é uma constante escolhida pelo projetista.

Neste trabalho, os problemas com incerteza nas forças são solucionados utilizando $f_{\sigma}^{\delta}\left(\bar{\rho}_{k}\right)$ com $\delta_{\sigma}=3$. O problema com incerteza de manufatura é solucionado utilizando $f_{\sigma}^{\varepsilon}\left(\bar{\rho}_{k}\right) \operatorname{com} \varepsilon=0.2$. Ambas as funções de interpolação utilizadas foram eficazes na relaxação das restrições de tensão, de forma que o método baseado em gradiente proposto na seção 6 foi utilizado com sucesso na obtenção de topologias $0 / 1$.

\subsection{Natureza local do critério de falha em tensão}

Idealmente, considerando um problema contínuo de otimização topológica, define-se a restrição de tensão sobre todos os pontos do domínio de projeto, de forma a garantir que nenhum ponto da estrutura otimizada apresente uma tensão de von Mises, por exemplo, que seja superior a tensão admissível imposta pelo projetista. Na prática, onde métodos de aproximação são utilizados para obter os deslocamentos e tensões, como o MEF, as tensões são definidas sobre pontos específicos no domínio de projeto, como o centroide de cada elemento finito, por exemplo (PEREIRA; FANCELLO; BARCELLOS, 2004). Desta forma, em vez de aplicar a restrição de tensão sobre todos os pontos do domínio, estas são impostas sobre um número finito de pontos.

Entretanto, ainda que o problema de otimização resultante (discretizado) tenha um número finito de restrições de tensão, este número é em geral muito alto, pois malhas bastante refinadas (com milhares de elementos) são usualmente necessárias para obter uma boa acurácia no cálculo de tensão (SILVA; BECK; SIGMUND, 2019).

Os métodos de otimização usualmente empregados na literatura de otimização topológica, como a programação linear sequencial (LAMBERTI; PAPPALETTERE, 2000), o método de linearização convexa (FLEURY, 1989) e o método das assíntotas móveis (SVANBERG, 1987), classificados por Duysinx, Bruyneel e Fleury (2009) como métodos de programação sequencial, não são apropriados para solucionar o problema com restrição de tensão de forma direta, ou seja, quando este é definido conforme apresentado na Equação (4.1). Métodos de programação sequencial são, em geral, bastante eficientes quando empregados em problemas de otimização com um grande número de variáveis de projeto e um pequeno número de restrições de projeto, o que usualmente ocorre na 
otimização topológica baseada em densidade (DUYSINX; BRUYNEEL; FLEURY, 2009). Problemas baseados em tensão apresentam, em geral, um grande número de variáveis de projeto e, ao mesmo tempo, um grande número de restrições de tensão, o que dificulta a sua solução com métodos de programação sequencial, devido a necessidade de se obter o gradiente de cada restrição separadamente (análise de sensibilidade local por meio da abordagem adjunta), o que aumenta de forma proibitiva o custo computacional (PARÍS et al., 2009).

De forma a superar a dificuldade relacionada ao grande número de restrições de tensão no problema de otimização, três abordagens são usualmente empregadas na literatura:

1. Utilização de técnicas de agregação, que consistem em funções que agregam todas as restrições locais em uma única restrição global, ou, alternativamente, em um conjunto de restrições regionais de tensão (DUYSINX; SIGMUND, 1998; HOLMBERG; TORSTENFELT; KLARBRING, 2013; KIYONO; SILVA; REDDY, 2016; LE et al., 2010; LEON et al., 2015; LONG; WANG; LIU, 2018; LUO; WANG; KANG, 2013; PEREIRA; CARDOSO, 2018; PARÍS et al., 2009; SVÄRD, 2015; TROYA; TORTORELLI, 2018; WANG; QIAN, 2018). O problema resultante, com uma única restrição, ou com um número reduzido de restrições, é solucionado com método de programação sequencial;

2. Considerar restrições $\epsilon$-ativas, onde cria-se uma estratégia para a consideração das restrições mais perigosas durante o processo de otimização (BRUGGI; DUYSINX, 2012; DUYSINX; BENDSØE, 1998). O problema é solucionado com método de programação sequencial, onde apenas algumas restrições são consideradas a cada passo do algoritmo, reduzindo o custo computacional associado a análise de sensibilidade local;

3. Lidar com o problema de forma direta, considerando todas as restrições locais de tensão, utilizando-se o método do Lagrangiano aumentado (FANCELLO, 2006; FANCELLO; PEREIRA, 2003; PEREIRA; CARDOSO, 2018; PEREIRA; FANCELLO; BARCELLOS, 2004). Neste caso, todas as restrições de tensão são agrupadas em um único somatório e adicionadas a função objetivo, o que permite a realização da análise de sensibilidade de todas elas de forma simultânea, reduzindo o custo computacional (FANCELLO; PEREIRA, 2003).

Segundo Duysinx e Sigmund (1998), París et al. (2009) e Pereira e Cardoso (2018), a utilização de abordagens locais (abordagens 2 ou 3) garante um melhor controle sobre o critério de falha em tensão. Conforme mostrado em Pereira e Cardoso (2018), a utilização de técnica de agregação (abordagem 1) fornece resultados dependentes do parâmetro de 
agregação utilizado, parâmetro o qual pode aumentar excessivamente a não linearidade da restrição global de tensão, gerando problemas de convergência. Por outro lado, deve-se salientar que diversos autores fazem o uso de técnicas de agregação (abordagem 1) e mostram bons resultados, como é o caso dos trabalhos recentes de Troya e Tortorelli (2018) e Wang e Qian (2018), onde problemas com centenas de milhares de restrições locais de tensão são solucionados empregando-se técnicas de agregação, e topologias com boa qualidade são obtidas. Desta forma, uma vez que não existe consenso na literatura sobre qual a melhor abordagem para lidar com o excessivo número de restrições de tensão, é de responsabilidade do projetista a escolha da abordagem mais adequada para a solução do problema em estudo.

Nesta tese, o método do Lagrangiano aumentado é utilizado (abordagem 3). A descrição do método e o algoritmo de solução são apresentados posteriormente na seção 6 .

\subsection{Cálculo da tensão no contorno irregular}

Conforme apresentado na seção 2, a otimização topológica baseada em densidade é uma abordagem de malha fixa, onde o contorno da topologia é dado de forma implícita. Em uma abordagem de malha fixa, as estruturas otimizadas usualmente apresentam topologias cujo contorno é descrito de forma irregular, ou seja, um contorno onde a malha de elementos finitos não está alinhada com o contorno da topologia subjacente. Conforme enfatizado por Svärd (2015), quando o contorno da estrutura não está alinhado com a malha de elementos finitos, concentrações artificiais de tensão que não possuem significado físico aparecem nos contornos irregulares, Figura 8. Conforme demonstrado por Svärd (2015), as concentrações artificiais de tensão no contorno irregular podem afetar de forma negativa a otimização, pois estas são geralmente diferentes das tensões da estrutura subjacente, de contorno suave.

Figura 8: Distribuição de tensão em estrutura com contorno irregular: (a) estrutura; (b) primeiras tensões principais avaliadas no centroide de cada elemento dado um carregamento de tensão uniaxial na barra.

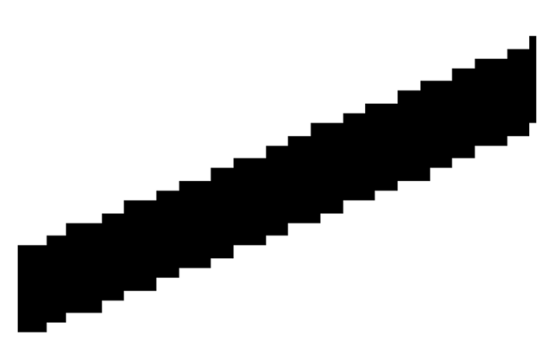

(a)

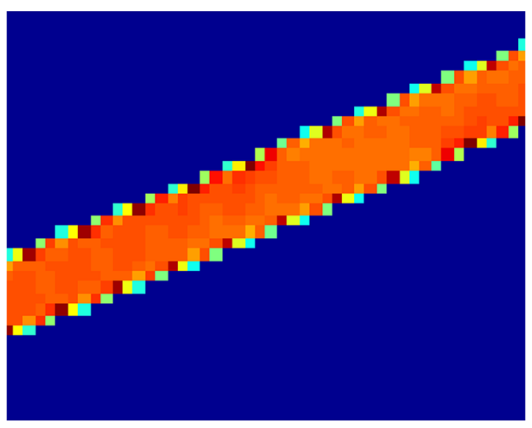

(b)

Fonte: adaptado de Svärd (2015).

O problema da avaliação de tensão em contorno irregular não é apenas uma 
dificuldade associada a métodos de malha fixa na otimização topológica. Este fenômeno já foi relatado em vários trabalhos relacionados a áreas diferentes (CHARRAS; GULDBERG, 2000; DOITRAND et al., 2015; FANG et al., 2016; GULDBERG; HOLLISTER; CHARRAS, 1998; HOLLISTER; RIEMER, 1993). Estes mesmos trabalhos apresentam técnicas que podem ser utilizadas para melhorar a acurácia no cálculo de tensão em contornos irregulares, e a maioria deles é baseada em uma etapa de pós-processamento de suavização de tensão. Entretanto, conforme mostrado em Doitrand et al. (2015), técnicas de suavização de tensão devem ser utilizadas com cautela, pois a acurácia do resultado fica dependente do refino da malha e do nível de suavização.

Na área de otimização topológica, pouca atenção tem sido dada a esta dificuldade. Até o momento, sabe-se da existência do IVE (Interior Value Extrapolation), que consiste em uma técnica desenvolvida recentemente por Svärd (2015), que extrapola as tensões do interior da estrutura (onde as tensões são calculadas com acurácia) para o seu contorno (SVÄRD, 2015). Svärd (2015) demonstra que o IVE funciona melhor do que a abordagem de avaliação de tensão tradicional para aproximar as tensões no contorno da estrutura subjacente. Entretanto, a formulação proposta por Svärd (2015), até o momento, não foi utilizada em outros trabalhos, na literatura.

Nesta tese, quatro formulações são apresentadas: três formulações sob incerteza nas forças e uma sob incerteza de manufatura. O efeito do contorno irregular não é abordado nas formulações sob incerteza nas forças. Deve-se estar ciente de que as estruturas otimizadas com estas abordagens devem ser pós-processadas e, caso uma estrutura inviável seja obtida (onde as tensões calculadas são maiores do que a tensão limite), estas devem ser otimizadas novamente por meio da utilização de métodos de otimização de forma.

Na formulação sob incerteza de manufatura, verificou-se que a acurácia na avaliação da tensão é de extrema importância e que o efeito de contornos irregulares não pode ser simplesmente ignorado. De forma a obter a acurácia necessária, ao invés de utilizar o IVE ou técnicas de suavização de tensão, utilizou-se a abordagem recentemente proposta por Silva, Beck e Sigmund (2019). Esta abordagem se baseia na consideração de uma fina camada de material intermediário entre a fase sólida e a fase vazia da topologia, associada a escolha adequada de parâmetros de interpolação de rigidez e tensão. Detalhes sobre a abordagem utilizada são apresentados posteriormente na subseção 5.2. 



\section{FORMULAÇÕES PROPOSTAS}

Nesta seção, quatro formulações, que abordam problemas de otimização topológica de minimização de volume com restrição de tensão sob incertezas, são propostas. A subseção 5.1 propõe três formulações que lidam com incerteza nas forças aplicadas. A subseção 5.2 propõe uma formulação que lida com incerteza uniforme de manufatura.

O método de solução utilizado para solucionar os problemas de otimização estrutural é descrito na seção 6 . A seção 5 se limita a apresentar os métodos de solução necessários para solucionar os problemas internos de otimização (análise de confiabilidade inversa e problemas de anti-otimização), problemas estes inerentes a algumas formulações.

\subsection{Minimização de volume com restrição de tensão e incerteza nas forças}

As formulações apresentadas nesta subseção são baseadas nas formulações apresentadas nas seções 3, de otimização sob incertezas, e 4, de otimização com restrição de tensão. A estrutura numérica das formulações baseadas em tensão sob incerteza nas forças, propostas neste trabalho, diferem da formulação baseada em tensão tradicional em dois pontos:

1. A inclusão de uma constante $\sigma_{\min }=1 \times 10^{-4} \sigma_{y}$ no cálculo da tensão equivalente de von Mises, como

$$
\sigma_{e q}^{(k)}(\overline{\boldsymbol{\rho}}, \mathbf{Q})=\sqrt{\boldsymbol{\sigma}_{k}^{T}(\overline{\boldsymbol{\rho}}, \mathbf{Q}) \mathbf{M} \boldsymbol{\sigma}_{k}(\overline{\boldsymbol{\rho}}, \mathbf{Q})+\sigma_{m i n}^{2}}
$$

onde $\mathbf{Q} \in \mathbb{R}^{N}$ representa um vetor de intensidades e/ou direções de forças incertas, particularizado para cada formulação posteriormente, e $\boldsymbol{\sigma}_{k}(\overline{\boldsymbol{\rho}}, \mathbf{Q})$ é a tensão interpolada, Equação (4.5). A constante $\sigma_{m i n}$ é incluída na implementação para evitar problemas numéricos durante a análise de sensibilidades, quando $\boldsymbol{\sigma}_{k}^{T}(\overline{\boldsymbol{\rho}}, \mathbf{Q}) \mathbf{M} \boldsymbol{\sigma}_{k}(\overline{\boldsymbol{\rho}}, \mathbf{Q}) \rightarrow 0$, pois a tensão aparece algumas vezes como denominador.

2. A utilização de uma versão penalizada do volume estrutural, $V_{p}(\overline{\boldsymbol{\rho}})=\sum_{e=1}^{N_{e}} V_{e} f_{v}\left(\bar{\rho}_{e}\right)$, onde $f_{v}\left(\bar{\rho}_{e}\right)$ é escolhida de forma a penalizar as densidades intermediárias, auxiliando a obtenção de topologias $0 / 1$. Nesta tese, utiliza-se $f_{v}\left(\bar{\rho}_{e}\right)=1-e^{-\delta_{v} \bar{\rho}_{e}}+\bar{\rho}_{e} e^{-\delta_{v}}$, a mesma função empregada para relaxação das restrições de tensão e projeção das densidades filtradas em um espaço $0 / 1$, com $\delta_{v}=5$. Apesar de apresentada em algumas formulações determinísticas como um recurso adicional, ou uma alternativa, na obtenção de topologias 0/1, como nos trabalhos de Le et al. (2010) e París et al. (2009), a penalização de volume não é muito comum na literatura de otimização topológica baseada em tensão. No desenvolvimento desta tese, no entanto, bons resultados foram obtidos com o uso deste recurso. 


\subsubsection{Formulação robusta probabilística}

Considera-se que o problema de minimização de volume com restrição de tensão pode estar sujeito a incertezas na intensidade e direção das forças aplicadas, definidas por variáveis aleatórias contidas em um vetor $\mathbf{Q}=\mathbf{Z}$. Cada componente $i$ do vetor de variáveis aleatórias $\mathbf{Z} \in \mathbb{R}^{N}$ representa uma intensidade ou direção incerta de uma força aplicada, de valor esperado $\mathrm{E}\left[Z_{i}\right]$ e desvio padrão $\operatorname{Std}\left[Z_{i}\right]$. Baseando-se nas Equações (3.2) e (4.1), escreve-se o problema robusto probabilístico como

$$
\begin{array}{cll}
\underset{\boldsymbol{\rho}}{\operatorname{minimizar}} & V_{p}(\overline{\boldsymbol{\rho}}) & \\
\text { sujeito a } & \frac{\hat{\sigma}_{e q}^{(k)}(\overline{\boldsymbol{\rho}}, \mathbf{Z})}{\sigma_{y}}-1 \leqslant 0 & k=1 . . N_{k} \\
& 0 \leqslant \rho_{e} \leqslant 1 & e=1 . . N_{e} \\
& \mathbf{K}(\overline{\boldsymbol{\rho}}) \mathbf{U}(\overline{\boldsymbol{\rho}}, \mathbf{Z})=\mathbf{F}(\mathbf{Z}) &
\end{array}
$$

onde

$$
\hat{\sigma}_{e q}^{(k)}(\overline{\boldsymbol{\rho}}, \mathbf{Z})=\mathrm{E}\left[\sigma_{e q}^{(k)}(\overline{\boldsymbol{\rho}}, \mathbf{Z})\right]+\alpha \operatorname{Std}\left[\sigma_{e q}^{(k)}(\overline{\boldsymbol{\rho}}, \mathbf{Z})\right]
$$

é uma medida de tensão robusta, calculada com base no valor esperado e no desvio padrão da tensão equivalente de von Mises. O valor esperado e a variância da tensão equivalente de von Mises, no ponto $k$, são calculados com o método de perturbação de primeira ordem, como

$$
\mathrm{E}\left[\sigma_{e q}^{(k)}(\overline{\boldsymbol{\rho}}, \mathbf{Z})\right]=\sigma_{e q}^{(k)}(\overline{\boldsymbol{\rho}}, \mathrm{E}[\mathbf{Z}])
$$

e

$$
\operatorname{Var}\left[\sigma_{e q}^{(k)}(\overline{\boldsymbol{\rho}}, \mathbf{Z})\right]=\sum_{i=1}^{N} \sum_{j=1}^{N}\left(\left.\frac{\partial \sigma_{e q}^{(k)}(\overline{\boldsymbol{\rho}}, \mathbf{z})}{\partial z_{i}}\right|_{\mathbf{z}=\mathrm{E}[\mathbf{Z}]}\right)\left(\left.\frac{\partial \sigma_{e q}^{(k)}(\overline{\boldsymbol{\rho}}, \mathbf{z})}{\partial z_{j}}\right|_{\mathbf{z}=\mathrm{E}[\mathbf{Z}]}\right) \operatorname{Cov}\left[Z_{i}, Z_{j}\right]
$$

O desvio padrão é obtido como

$$
\operatorname{Std}\left[\sigma_{e q}^{(k)}(\overline{\boldsymbol{\rho}}, \mathbf{Z})\right]=\sqrt{\operatorname{Var}\left[\sigma_{e q}^{(k)}(\overline{\boldsymbol{\rho}}, \mathbf{Z})\right]+\sigma_{m i n}^{2}}
$$

onde a constante $\sigma_{\min }$ é incluída na formulação para evitar problemas numéricos durante a análise de sensibilidades, quando $\operatorname{Var}\left[\sigma_{e q}^{(k)}(\overline{\boldsymbol{\rho}}, \mathbf{Z})\right] \rightarrow 0$, pois o desvio padrão, tal como o valor esperado da tensão, Equação (5.4), aparece algumas vezes como denominador.

Note que o valor esperado da tensão equivalente de von Mises é a própria tensão avaliada no valor médio de $\mathbf{Z}$. A variância, por sua vez, e consequentemente o desvio padrão, necessitam das derivadas da tensão em relação às variáveis aleatórias, $\frac{\partial \sigma_{e q}^{(k)}(\overline{\boldsymbol{\rho}}, \mathbf{z})}{\partial z_{i}}$, para $i=1 . . N$, também avaliadas em $\mathbf{z}=\mathrm{E}[\mathbf{Z}]$.

De forma a obter o valor esperado e a variância da tensão equivalente de von Mises de forma sistemática, utilizam-se a equação de equilíbrio e suas derivadas em relação às 
variáveis aleatórias. A equação de equilíbrio avaliada no valor médio das forças externas, $\mathbf{F}(\mathrm{E}[\mathbf{Z}])=\mathbf{F}^{0}$, é dada por

$$
\mathbf{K}(\overline{\boldsymbol{\rho}}) \mathbf{U}^{0}(\overline{\boldsymbol{\rho}})=\mathbf{F}^{0},
$$

onde $\mathbf{U}^{0}(\overline{\boldsymbol{\rho}})$ é o valor médio dos deslocamentos nodais.

As derivadas da equação de equilíbrio em relação às variáveis aleatórias $\mathbf{Z}$ são dadas por

$$
\mathbf{K}(\overline{\boldsymbol{\rho}}) \mathbf{U}_{i}^{I}(\overline{\boldsymbol{\rho}})=\mathbf{F}_{i}^{I},
$$

onde $\mathbf{F}_{i}^{I}=\left.\frac{\partial \mathbf{F}(\mathbf{z})}{\partial z_{i}}\right|_{\mathbf{z}=\mathrm{E}[\mathbf{Z}]}$ é a derivada do vetor de forças externas em relação a variável aleatória $Z_{i}$, avaliada no valor médio de $\mathbf{Z}$; esta derivada é obtida de forma analítica pelo projetista e utilizada como dado de entrada no problema de otimização topológica. Esta equação é utilizada para obter $\mathbf{U}_{i}^{I}(\overline{\boldsymbol{\rho}})=\left.\frac{\partial \mathbf{U}(\overline{\boldsymbol{\rho}}, \mathbf{z})}{\partial z_{i}}\right|_{\mathbf{z}=\mathrm{E}[\mathbf{Z}]}$, derivada do vetor global de deslocamentos. Note que, de forma a solucionar as Equações (5.7) e (5.8), para $i=1 . . N$, pode-se efetuar a fatoração da matriz de rigidez $\mathbf{K}(\overline{\boldsymbol{\rho}})$ uma única vez (para uma dada distribuição de densidades relativas $\overline{\boldsymbol{\rho}}$ ), pois a mesma matriz é utilizada em ambas as equações.

Substituindo (4.3) em (4.5), e (4.5) em (5.1), pode-se escrever a tensão equivalente de von Mises como

$$
\sigma_{e q}^{(k)}(\overline{\boldsymbol{\rho}}, \mathbf{Z})=\sqrt{\mathbf{u}_{k}^{T}(\overline{\boldsymbol{\rho}}, \mathbf{Z}) \mathbf{B}_{k}^{T} \overline{\mathbf{C}}\left(\bar{\rho}_{k}\right) \mathbf{M} \overline{\mathbf{C}}\left(\bar{\rho}_{k}\right) \mathbf{B}_{k} \mathbf{u}_{k}(\overline{\boldsymbol{\rho}}, \mathbf{Z})+\sigma_{m i n}^{2}}
$$

onde $\overline{\mathbf{C}}\left(\bar{\rho}_{k}\right)=f_{\sigma}\left(\bar{\rho}_{k}\right) \mathbf{C}^{b}$ consiste no tensor constitutivo do material base multiplicado pela função de interpolação de tensão, definido por praticidade.

O valor esperado da tensão equivalente de von Mises é a própria tensão avaliada no valor médio de $\mathbf{Z}$, Equação (5.4), tal que

$$
\mathrm{E}\left[\sigma_{e q}^{(k)}(\overline{\boldsymbol{\rho}}, \mathbf{Z})\right]=\sqrt{\left[\mathbf{u}_{k}^{0}(\overline{\boldsymbol{\rho}})\right]^{T} \mathbf{B}_{k}^{T} \overline{\mathbf{C}}\left(\bar{\rho}_{k}\right) \mathbf{M} \overline{\mathbf{C}}\left(\bar{\rho}_{k}\right) \mathbf{B}_{k} \mathbf{u}_{k}^{0}(\overline{\boldsymbol{\rho}})+\sigma_{m i n}^{2}},
$$

onde $\mathbf{u}_{k}^{0}(\overline{\boldsymbol{\rho}})=\mathbf{H}_{k} \mathbf{U}^{0}(\overline{\boldsymbol{\rho}})$.

Para calcular a variância da tensão de von Mises, deve-se primeiro obter a sua derivada em relação às variáveis aleatórias. A derivada de $\sigma_{e q}^{(k)}(\overline{\boldsymbol{\rho}}, \mathbf{Z})$, Equação (5.9), em relação a uma variável aleatória é dada por

$$
\left.\frac{\partial \sigma_{e q}^{(k)}(\overline{\boldsymbol{\rho}}, \mathbf{z})}{\partial z_{i}}\right|_{\mathbf{z}=\mathrm{E}[\mathbf{Z}]}=\frac{\left[\mathbf{u}_{k}^{0}(\overline{\boldsymbol{\rho}})\right]^{T} \mathbf{B}_{k}^{T} \overline{\mathbf{C}}\left(\bar{\rho}_{k}\right) \mathbf{M} \overline{\mathbf{C}}\left(\bar{\rho}_{k}\right) \mathbf{B}_{k}\left(\mathbf{u}_{k}\right)_{i}^{I}(\overline{\boldsymbol{\rho}})}{\mathrm{E}\left[\sigma_{e q}^{(k)}(\overline{\boldsymbol{\rho}}, \mathbf{Z})\right]}
$$

onde $\left(\mathbf{u}_{k}\right)_{i}^{I}(\overline{\boldsymbol{\rho}})=\mathbf{H}_{k} \mathbf{U}_{i}^{I}(\overline{\boldsymbol{\rho}})$. A variância, e posteriormente o desvio padrão, são calculados substituindo (5.11) em (5.5), e (5.5) em (5.6). Note que os cálculos necessários para avaliar o valor esperado, Equação (5.10), e a derivada da tensão equivalente de von Mises, Equação 
(5.11), são cálculos locais e dependem unicamente dos vetores globais de deslocamento $\mathbf{U}^{0}(\overline{\boldsymbol{\rho}})$ e $\mathbf{U}_{i}^{I}(\overline{\boldsymbol{\rho}})$, para $i=1 . . N$, obtidos a partir da solução das equações de equilíbrio (5.7) e (5.8), respectivamente.

A formulação descrita nesta subseção é apresentada em detalhes, também, em Silva, Beck e Cardoso (2018), que consiste em um trabalho recente do autor e fruto desta tese.

\subsubsection{Formulação baseada em confiabilidade}

Considera-se que o problema de minimização de volume com restrição de tensão pode estar sujeito a incertezas na intensidade e direção das forças aplicadas, definidas por variáveis aleatórias contidas em um vetor $\mathbf{Q}=\mathbf{Z}$. O problema baseado em confiabilidade é formulado com o PMA, como

$$
\begin{array}{cll}
\underset{\boldsymbol{\rho}}{\operatorname{minimizar}} & V_{p}(\overline{\boldsymbol{\rho}}) \\
\text { sujeito a } & \frac{\sigma_{e q}^{(k)}\left(\overline{\boldsymbol{\rho}},\left(\mathbf{Y}^{(k)}\right)^{*}\right)}{\sigma_{y}}-1 \leqslant 0 & k=1 . . N_{k} \\
& 0 \leqslant \rho_{e} \leqslant 1 & e=1 . . N_{e} \\
& \mathbf{K}(\overline{\boldsymbol{\rho}}) \mathbf{U}(\overline{\boldsymbol{\rho}}, \mathbf{Y})=\mathbf{F}(\mathbf{Y}) &
\end{array}
$$

onde as tensões equivalentes de von Mises são avaliadas nos pontos de mínimo desempenho $\left(\mathbf{Y}^{(k)}\right)^{*}$, obtidos por meio de uma análise de confiabilidade inversa no espaço normal padrão para cada $k$, como

$$
\begin{array}{ll}
\underset{\mathbf{Y}}{\operatorname{minimizar}} & -\sigma_{e q}^{(k)}(\overline{\boldsymbol{\rho}}, \mathbf{Y}) \\
\text { sujeito a } & \|\mathbf{Y}\|=\beta_{T}
\end{array}
$$

Note que um mapeamento de $\mathbb{Z} \rightarrow \mathbb{Y}$ é necessário neste caso, ou seja, do espaço de projeto $\mathbb{Z}$ para o espaço normal padrão $\mathbb{Y}$. Nesta tese, todas as variáveis aleatórias contidas em $\mathbf{Z}$ são consideradas Gaussianas, de forma que a transformação Hasofer e Lind é suficiente (LOPEZ; BECK, 2012). No entanto, quando variáveis aleatórias com outras distribuições são consideradas, outras técnicas de mapeamento devem ser utilizadas, técnicas as quais estão além do escopo desta tese e não são apresentadas. O leitor pode consultar Ditlevsen e Madsen (2007) para detalhes sobre estas técnicas de mapeamento.

Os problemas internos de otimização, Equação (5.13), podem ser solucionados por meio da utilização de qualquer algoritmo que seja capaz de lidar com problemas de otimização sujeitos a restrições de igualdade. Neste trabalho, estes problemas são solucionados com o método HMV (Hybrid Mean Value), conforme apresentado em Youn, Choi e Park (2003). Este é um algoritmo muito simples, que foi desenvolvido especificamente para solucionar problemas de análise de confiabilidade inversa. Apesar de fácil implementação, este algoritmo é muito robusto e de rápida convergência (YOUN; CHOI; PARK, 2003). 
O método HMV consiste em uma abordagem iterativa: para cada problema interno $k$, inicia-se em $\mathbf{Y}_{(j=0)}=\mathbf{0}$, onde $j$ indica a iteração do problema interno, com o objetivo de obter $\left(\mathbf{Y}^{(k)}\right)^{*}$, a solução do problema interno. No início de cada iteração $j$, calcula-se o negativo do gradiente da função objetivo do problema interno, como

$$
\mathbf{n}_{(j)}=-\frac{\nabla_{\mathbf{Y}}\left[-\sigma_{e q}^{(k)}\left(\overline{\boldsymbol{\rho}}, \mathbf{Y}_{(j)}\right)\right]}{\left\|\nabla_{\mathbf{Y}}\left[-\sigma_{e q}^{(k)}\left(\overline{\boldsymbol{\rho}}, \mathbf{Y}_{(j)}\right)\right]\right\|}=\frac{\nabla_{\mathbf{Y}} \sigma_{e q}^{(k)}\left(\overline{\boldsymbol{\rho}}, \mathbf{Y}_{(j)}\right)}{\left\|\nabla_{\mathbf{Y}} \sigma_{e q}^{(k)}\left(\overline{\boldsymbol{\rho}}, \mathbf{Y}_{(j)}\right)\right\|}
$$

O gradiente normalizado, Equação (5.14), é utilizado para obter $\mathbf{Y}_{(j+1)}$ a cada passo do problema interno de otimização. Para $j=0,1,2$, calcula-se $\mathbf{Y}_{(j+1)}=\beta_{T} \mathbf{n}_{(j)}$. Para $j \geqslant 3$, calcula-se um parâmetro adicional $\zeta_{(j)}$, após o cálculo de $\mathbf{n}_{(j)}$, de forma a escolher adequadamente o vetor $\mathbf{Y}_{(j+1)}$, como $\zeta_{(j)}=\left(\mathbf{n}_{(j)}-\mathbf{n}_{(j-1)}\right) \cdot\left(\mathbf{n}_{(j-1)}-\mathbf{n}_{(j-2)}\right)$.

Se $\zeta_{(j)}>0$, então

$$
\mathbf{Y}_{(j+1)}=\beta_{T} \mathbf{n}_{(j)}
$$

caso contrário

$$
\mathbf{Y}_{(j+1)}=\beta_{T} \frac{\mathbf{n}_{(j)}+\mathbf{n}_{(j-1)}+\mathbf{n}_{(j-2)}}{\left\|\mathbf{n}_{(j)}+\mathbf{n}_{(j-1)}+\mathbf{n}_{(j-2)}\right\|} .
$$

O algoritmo é aplicado até que uma tolerância especificada pelo projetista seja obtida (YOUN; CHOI; PARK, 2003). Nesta tese, utiliza-se um simples critério sobre as variáveis de projeto dos problemas internos de otimização, $\mathbf{Y}$, como critério de convergência: $\max \left|\mathbf{Y}_{(j+1)}-\mathbf{Y}_{(j)}\right|<t_{\text {ol }}$, onde tol $_{Y}$ é uma tolerância especificada pelo projetista.

O gradiente da tensão equivalente de von Mises em relação às variáveis Y é obtido de forma analítica, por meio da derivada da Equação (5.9), para $\mathbf{Z}=\mathbf{Y}$, da mesma forma como feito anteriormente para o caso robusto probabilístico, Equação (5.11), considerando no entanto que a derivada é avaliada em $\mathbf{Y}_{(j)}$. A $i$-ésima componente de $\nabla_{\mathbf{Y}} \sigma_{e q}^{(k)}\left(\overline{\boldsymbol{\rho}}, \mathbf{Y}_{(j)}\right)$ é dada por

$$
\left.\frac{\partial \sigma_{e q}^{(k)}(\overline{\boldsymbol{\rho}}, \mathbf{y})}{\partial y_{i}}\right|_{\mathbf{y}=\mathbf{Y}_{(j)}}=\left.\frac{\mathbf{u}_{k}^{T}\left(\overline{\boldsymbol{\rho}}, \mathbf{Y}_{(j)}\right) \mathbf{B}_{k}^{T} \overline{\mathbf{C}}\left(\bar{\rho}_{k}\right) \mathbf{M} \overline{\mathbf{C}}\left(\bar{\rho}_{k}\right) \mathbf{B}_{k}}{\sigma_{e q}^{(k)}\left(\overline{\boldsymbol{\rho}}, \mathbf{Y}_{(j)}\right)} \frac{\partial \mathbf{u}_{k}(\overline{\boldsymbol{\rho}}, \mathbf{y})}{\partial y_{i}}\right|_{\mathbf{y}=\mathbf{Y}_{(j)}} .
$$

A solução dos problemas internos de otimização exige, muitas vezes, a avaliação do gradiente $\nabla_{\mathbf{Y}} \sigma_{e q}^{(k)}\left(\overline{\boldsymbol{\rho}}, \mathbf{Y}_{(j)}\right)$ diversas vezes durante a aplicação do método HMV. Em problemas baseados em tensão, malhas bastante refinadas são usualmente necessárias, o que implica em um elevado número de pontos de avaliação de tensão e, consequentemente, em um elevado número de problemas internos de otimização, Equação (5.13), a serem solucionados a cada passo da solução do problema externo de otimização topológica, Equação (5.12).

Para reduzir o custo computacional associado a avaliação de $\nabla_{\mathbf{Y}} \sigma_{e q}^{(k)}\left(\overline{\boldsymbol{\rho}}, \mathbf{Y}_{(j)}\right)$, podese utilizar o princípio da superposição de efeitos, válido para a hipótese de elasticidade 
linear. Conforme descrito em Silva e Beck (2018), para problemas sujeitos a forças de direção determinística e intensidade incerta, pode-se fazer o uso de vetores globais de deslocamentos auxiliares (um para cada força incerta); estes são obtidos por meio da solução da equação de equilíbrio para forças de intensidade unitária com mesma direção das forças originais. Os deslocamentos locais devido a uma realização qualquer, $\mathbf{u}_{k}\left(\overline{\boldsymbol{\rho}}, \mathbf{Y}_{(j)}\right)$, necessários para calcular $\nabla_{\mathbf{Y}} \sigma_{e q}^{(k)}\left(\overline{\boldsymbol{\rho}}, \mathbf{Y}_{(j)}\right)$, são então obtidos de forma explícita, por meio de cálculos locais sobre os vetores de deslocamento auxiliares (SILVA; BECK, 2018). Esta estratégia foi estendida para lidar com incerteza na direção das forças em Silva, Cardoso e Beck (2018), onde propôs-se a utilização de duas forças (em 2D) de intensidade unitária, uma vertical e uma horizontal, para cada direção incerta. Neste caso, consideram-se dois vetores globais de deslocamentos auxiliares para cada direção incerta.

Note que a formulação baseada em confiabilidade apresentada nas Equações (5.12) e (5.13) impõe uma restrição de confiabilidade sobre a tensão equivalente de von Mises em cada ponto de cálculo de tensão, o que pode ser visto como uma extensão natural da formulação determinística, Equação (4.1). Do ponto de vista de confiabilidade estrutural, no entanto, seria mais significativo trabalhar com uma única restrição sobre a confiabilidade do sistema ao invés disso. O problema de otimização considerando a confiabilidade de sistemas é abordado por alguns autores, na literatura (AOUES; CHATEAUNEUF, 2008; BA-ABBAD; NIKOLAIDIS; KAPANIA, 2006; LIANG; MOURELATOS; NIKOLAIDIS, 2007; NGUYEN; SONG; PAULINO, 2009), mas aplicações destas técnicas em problemas contínuos com restrição de tensão ainda são inexistentes. O problema baseado em tensão com restrição sobre a confiabilidade do sistema ainda é um problema em aberto, na literatura, e não é abordado nesta tese.

A formulação descrita nesta subseção é apresentada em detalhes, também, em Silva e Beck (2018), que consiste em um trabalho recente do autor e fruto desta tese.

\subsubsection{Formulação robusta não probabilística}

Considera-se que o problema de minimização de volume com restrição de tensão pode estar sujeito a incertezas na intensidade e direção das forças aplicadas, definidas por variáveis incertas-mas-limitadas contidas em um vetor $\mathbf{Q}=\mathbf{W}$. O problema robusto não probabilístico é formulado como um problema de otimização com anti-otimização, como

$$
\begin{array}{cll}
\underset{\boldsymbol{\rho}}{\operatorname{minimizar}} & V_{p}(\overline{\boldsymbol{\rho}}) & \\
\text { sujeito a } & \frac{\sigma_{e q}^{(k)}\left(\overline{\boldsymbol{\rho}},\left(\mathbf{W}^{(k)}\right)^{*}\right)}{\sigma_{y}}-1 \leqslant 0 & k=1 . . N_{k} \\
& 0 \leqslant \rho_{e} \leqslant 1 & e=1 . . N_{e} \\
& \mathbf{K}(\overline{\boldsymbol{\rho}}) \mathbf{U}(\overline{\boldsymbol{\rho}}, \mathbf{W})=\mathbf{F}(\mathbf{W}) &
\end{array}
$$

onde as tensões equivalentes de von Mises são avaliadas em $\left(\mathbf{W}^{(k)}\right)^{*}$ (pontos que fornecem as tensões máximas de von Mises), obtidos por meio de uma análise de pior cenário para 
cada $k$, como

$$
\begin{array}{ll}
\underset{\mathbf{W}}{\operatorname{maximizar}} & \sigma_{e q}^{(k)}(\boldsymbol{\rho}, \mathbf{W}) \\
\text { sujeito a } & \underline{\mathbf{W}} \leqslant \mathbf{W} \leqslant \overline{\mathbf{W}}
\end{array}
$$

Conforme discutido anteriormente na subseção 3.3, os pontos obtidos como solução dos problemas de anti-otimização, $\left(\mathbf{W}^{(k)}\right)^{*}$, para $k=1 . . N_{k}$, devem ser ótimos globais, de forma a garantir a robustez da solução do problema de otimização. Os problemas de anti-otimização obtidos com a formulação baseada em tensão, Equação (5.19), no entanto, são em geral não convexos; e garantir ótimo global em problemas de otimização não convexos é em geral uma tarefa muito desafiadora (ARORA, 2012).

Neste trabalho, os problemas de anti-otimização são solucionados com uma abordagem sequencial de duas etapas: 1) uma abordagem de busca em grade (RAO, 2009) é inicialmente empregada; 2) partindo do ponto que fornece a maior tensão equivalente de von Mises obtido com a busca em grade, emprega-se um método de descida mais íngreme modificado (aqui subida, pois a tensão máxima é desejada), seguindo algoritmo proposto na subseção 6.2, até que a tolerância especificada pelo projetista seja alcançada. Neste trabalho, utiliza-se uma simples tolerância sobre as variáveis de projeto, $\max \left|\mathbf{W}_{(j+1)}-\mathbf{W}_{(j)}\right|<$ tol $_{W}$, onde $j$ representa a iteração do problema de anti-otimização. A saída da abordagem sequencial de duas etapas é considerada solução do problema de anti-otimização. Note que, para aplicar a segunda etapa com o método sugerido de descida mais íngreme modificado, deve-se obter o gradiente da tensão equivalente de von Mises em relação a W. Este gradiente é obtido da mesma forma como mostrado anteriormente para o problema baseado em confiabilidade, Equação (5.17), considerando $\mathbf{W}$ em vez de $\mathbf{Y}$.

Obviamente, não existe garantia de que o ponto obtido desta abordagem sequencial é um ponto de ótimo global. Entretanto, os resultados numéricos apresentados na seção 7 mostram que soluções verdadeiramente robustas em relação às incertezas nas forças aplicadas são obtidas com o uso desta abordagem.

Além disso, a formulação proposta neste trabalho é bastante geral, de forma que o algoritmo de solução empregado para solucionar os problemas de anti-otimização pode ser substituído, sem perda de generalidade. Uma alternativa é a utilização de algoritmos que garantem a obtenção de ótimo global dada uma tolerância especificada pelo projetista. Thore (2016), por exemplo, propõe um algoritmo do tipo branch-and-bound, de forma a obter o valor máximo (ótimo global) da norma de tensão, dadas forças externas incertasmas-limitadas.

A formulação descrita nesta subseção é apresentada em detalhes, também, em Silva, Cardoso e Beck (2018), que consiste em um trabalho recente do autor e fruto desta tese. 


\subsection{Minimização de volume com restrição de tensão e incerteza de manufatura}

A formulação apresentada nesta subseção é baseada na abordagem robusta não probabilística descrita por Wang, Lazarov e Sigmund (2011). Esta abordagem difere da formulação não probabilística de otimização com anti-otimização tradicional, Equações (5.18) e (5.19), e foi desenvolvida especificamente para lidar com incertezas de manufatura em problemas contínuos de otimização topológica. Esta abordagem consiste na consideração simultânea de três campos de densidades relativas durante o procedimento de otimização: erodido $\left(\overline{\boldsymbol{\rho}}^{(e)}\right)$, intermediário $\left(\overline{\boldsymbol{\rho}}^{(i)}\right)$ e dilatado $\left(\overline{\boldsymbol{\rho}}^{(d)}\right)$; campos os quais são definidos com a Equação (2.11) para três valores distintos de $\eta$, sendo eles: $\eta_{e}, \eta_{i}$ e $\eta_{d}$, respectivamente; com $\eta_{e}>\eta_{i}>\eta_{d}$. A ideia principal da abordagem robusta de três campos consiste na redução da sensibilidade da topologia intermediária $\left(\overline{\boldsymbol{\rho}}^{(i)}\right)$ a incertezas uniformes de manufatura, por meio da inclusão dos campos erodido e dilatado na formulação do problema de otimização (WANG; LAZAROV; SIGMUND, 2011). Nesta tese, onde o problema de minimização de volume com restrição local de tensão é considerado, aplicam-se as restrições de projeto também sobre os campos adicionais, ou seja, além das restrições locais de tensão sobre a topologia intermediária, aplicam-se as restrições de tensão sobre as topologias erodida e dilatada. Como as topologias erodida, intermediária e dilatada são obtidas do mesmo campo de densidades filtradas, Equação (2.8), espera-se que estas sejam as mesmas no final do procedimento de otimização, garantindo topologias mais finas (erodidas) e mais grossas (dilatadas) do que a topologia intermediária, e que também satisfaçam o critério de falha local em tensão.

O problema de minimização de volume com restrição de tensão considerando incerteza uniforme de manufatura é dado por

$$
\begin{array}{cll}
\underset{\boldsymbol{\rho}}{\operatorname{minimizar}} & V\left(\overline{\boldsymbol{\rho}}^{(d)}\right) & \\
\text { sujeito a } & \frac{\sigma_{e q}^{(k)}\left(\overline{\boldsymbol{\rho}}^{(e)}\right)}{\sigma_{y}}-1 \leqslant 0 & k=1 . . N_{k} \\
& \frac{\sigma_{e q}^{(k)}\left(\overline{\boldsymbol{\rho}}^{(i)}\right)}{\sigma_{y}}-1 \leqslant 0 & k=1 . . N_{k} \\
& \frac{\sigma_{e q}^{(k)}\left(\overline{\boldsymbol{\rho}}^{(d)}\right)}{\sigma_{y}}-1 \leqslant 0 & k=1 . . N_{k} \\
& 0 \leqslant \rho_{e} \leqslant 1 & e=1 . . N_{e} \\
& \mathbf{K}\left(\overline{\boldsymbol{\rho}}^{(e)}\right) \mathbf{U}\left(\overline{\boldsymbol{\rho}}^{(e)}\right)=\mathbf{F} & \\
& \mathbf{K}\left(\overline{\boldsymbol{\rho}}^{(i)}\right) \mathbf{U}\left(\overline{\boldsymbol{\rho}}^{(i)}\right)=\mathbf{F} & \\
& \mathbf{K}\left(\overline{\boldsymbol{\rho}}^{(d)}\right) \mathbf{U}\left(\overline{\boldsymbol{\rho}}^{(d)}\right)=\mathbf{F} & \\
&
\end{array}
$$

onde o volume da estrutura dilatada, o maior entre todos os volumes, é minimizado, de forma a garantir a minimização simultânea do volume das topologias intermediária e erodida, e evitar as instabilidades numéricas que surgem quando o volume da estrutura intermediária é considerada na formulação (SIGMUND, 2009). 
Conforme demonstrado na sequência, quando uma abordagem de malha fixa é utilizada para otimização topológica (como nesta tese), concentrações artificiais de tensão aparecem nos contornos irregulares, como descrito por Svärd (2015) e também enfatizado na subseção 4.3, gerando fortes oscilações e baixa acurácia no cálculo da tensão após variações uniformes de contorno e, consequentemente, soluções não robustas a incertezas de manufatura. Um dos principais objetivos desta subseção (5.2) é demonstrar que acurácia na avaliação de tensão e baixa oscilação de tensão após variação uniforme de contorno podem ser obtidas com escolhas razoáveis de funções de interpolação de rigidez e tensão e controle da quantidade de material intermediário entre as fases sólida e vazia da topologia, como discutido posteriormente nas subseções 5.2.2 e 5.2.3.

A principal diferença entre a abordagem robusta não probabilística descrita nesta subseção, Equação (5.20), e a abordagem determinística, Equação (4.1), está na solução de dois problemas de equilíbrio adicionais a cada etapa na solução do problema de otimização (WANG; LAZAROV; SIGMUND, 2011), e na consideração de um maior número de restrições de tensão (três vezes maior). Por exemplo, considerando um ponto de cálculo de tensão por elemento finito, tem-se três restrições de tensão por elemento. Considerando uma malha com 10k elementos, tem-se um problema de otimização com 10k variáveis de projeto e 30k restrições de tensão.

A formulação descrita nesta subseção, tal como as análises feitas nas subseções 5.2.1, 5.2.2 e 5.2.3, é apresentada em detalhes, também, em Silva, Beck e Sigmund (2019), que consiste em um trabalho recente do autor e fruto desta tese.

\subsubsection{Análise: chapa com furo circular}

Na área de otimização topológica, o problema da avaliação de tensão em contorno irregular já foi demonstrado por Svärd (2015), onde a acurácia do cálculo da tensão foi investigada para rotação de uma estrutura em uma malha fixa de elementos finitos, considerando diferentes tamanhos de malha. Entretanto, como a formulação proposta nesta subseção visa a obtenção de estruturas robustas a incertezas de manufatura, a investigação da acurácia da tensão para variações uniformes no contorno da topologia (erosão ou dilatação) é fundamental.

Nesta subseção, o problema da avaliação de tensão no contorno irregular é demonstrado por meio da utilização de um modelo de elementos finitos baseado em pixel (em forma de grade, com todos os elementos quadrados) para solucionar o problema bem conhecido da chapa com furo circular (PILKEY, 2007). O problema é discretizado como uma topologia $0 / 1$, simulando uma topologia ideal composta de sólidos e vazios: $\bar{\rho}=0$ quando o centroide do elemento está dentro do furo circular; e $\bar{\rho}=1$ caso contrário. A Figura 9 ilustra o problema analisado e o procedimento de discretização empregado.

O modelo baseado em pixel é discretizado com elementos bilineares isoparamétricos 
Figura 9: Problema da chapa com furo circular: (a) domínio completo e condições de contorno; (b) 1/4 do domínio após aplicação das condições de simetria; (c) modelo discretizado com o MEF baseado em pixel.

(a)

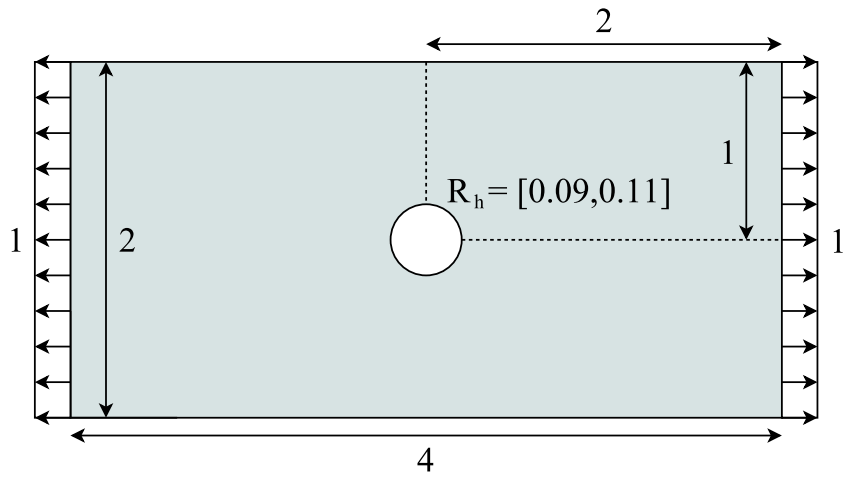

(b)

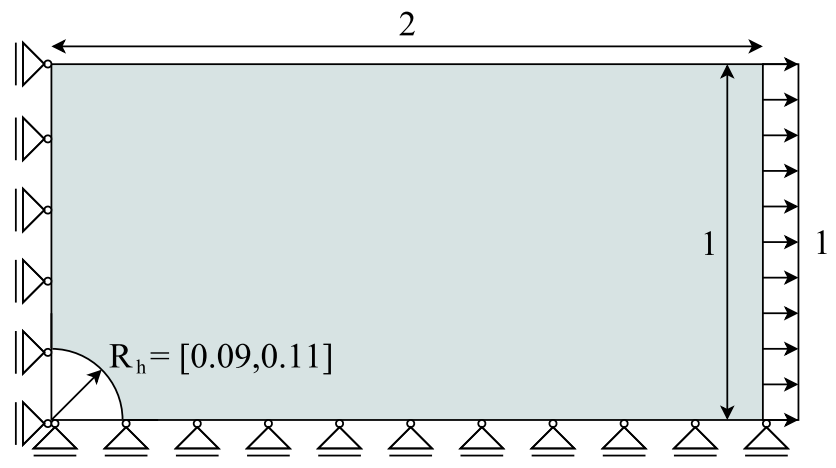

(c)
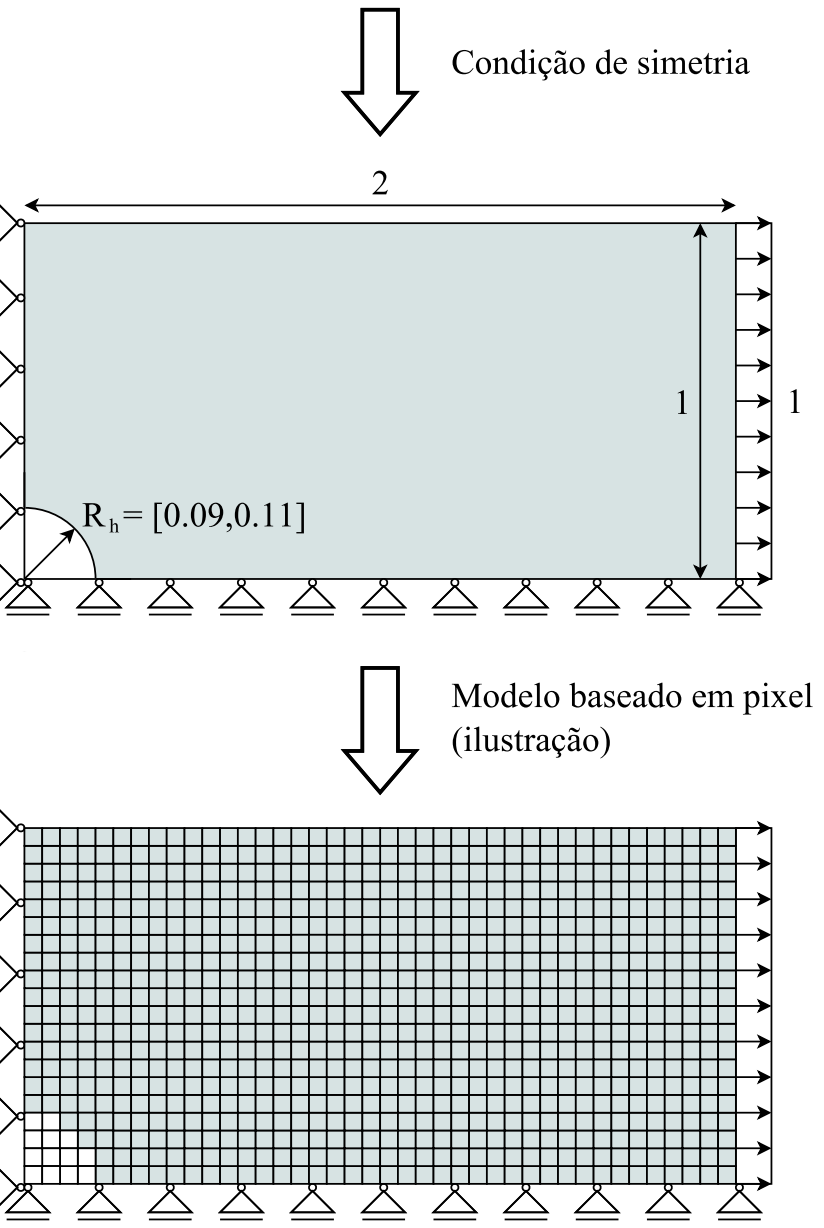

Fonte: adaptado de Silva, Beck e Sigmund (2019).

de quatro nós e as tensões são calculadas no centroide de cada elemento. Dados de entrada: módulo de elasticidade 1, espessura 1, coeficiente de Poisson 0.0. Avalia-se o valor máximo da componente de tensão $\sigma_{11}$ com o modelo baseado em pixel, Figura 10, para três malhas diferentes. $\mathrm{O}$ raio do furo circular $R_{h}$ é variado de 0.09 até 0.11 , simulando uma tolerância de manufatura.

A Figura 10 mostra claramente que o valor máximo de $\sigma_{11}$ superestima a solução 
Figura 10: Tensão máxima $\sigma_{11}$ calculada com três malhas distintas e comparada com a solução de referência ( $\cong 3$, linha cheia). O tamanho das legendas horizontais representa o tamanho dos elementos (lado dos quadrados).

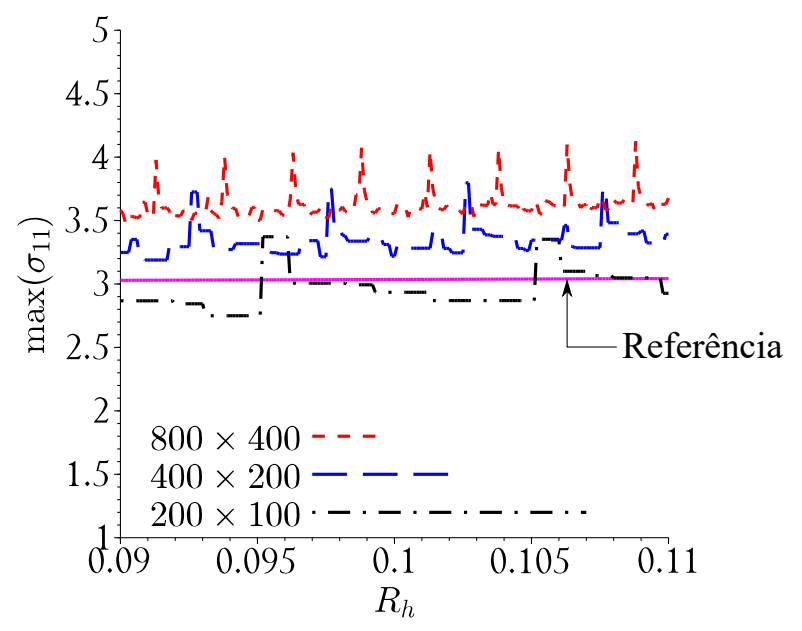

Fonte: adaptado de Silva, Beck e Sigmund (2019).

de referência com o refino da malha. Além disso, verifica-se que a resposta de tensão oscila fortemente com a variação do valor de $R_{h}$, ou seja, com uma variação de contorno uniforme, ao contrário do que acontece com a solução de referência, que é absolutamente suave. Verifica-se, também, que o refino da malha não ajuda a reduzir as oscilações na tensão máxima.

As subseções 5.2.2 e 5.2.3, apresentadas a seguir, mostram uma técnica simples e efetiva, que é utilizada para reduzir as oscilações no cálculo da tensão devido a variações uniformes no contorno, e melhorar a sua acurácia. Esta abordagem se baseia na: 1) consideração de uma fina camada de material intermediário entre a fase sólida e a fase vazia da topologia; e 2) na escolha adequada do parâmetro $\varepsilon$ para interpolação de tensão.

\subsubsection{Limitando a nitidez dos campos projetados}

Verifica-se, na Figura 10, que o comportamento oscilatório da tensão máxima tem periodicidade igual ao tamanho do elemento. Nesta subseção, a primeira etapa de um simples procedimento para suavização de tensão é apresentada, baseada na consideração de uma fina transição de material intermediário entre as fases sólida e vazia da topologia, transição a qual possui largura igual ou ligeiramente maior que o tamanho do elemento, ou seja, a distância entre dois picos de tensão consecutivos.

O procedimento aqui adotado para garantir uma fina transição de material intermediário entras as fases sólida e vazia consiste em: 1) aplicar o filtro de densidades, Equação (2.8), sobre o problema 0/1;2) aplicar a função de projeção, Equação (2.11), considerando um valor limite para $\delta$, de forma a limitar a nitidez do campo projetado. Quando o valor de $\delta$ é limitado, a inclinação da função de projeção é automaticamente limitada, ou seja, 
quanto maior o valor de $\delta$, menor é a largura da transição de material intermediário entre as fases sólida e vazia da topologia. Esta subseção visa demonstrar como o valor de $\delta$ pode ser limitado de forma a garantir uma transição de material intermediário entre as fases sólida e vazia com largura igual ou ligeiramente maior que o tamanho do elemento.

Considera-se um domínio contínuo unidimensional, com $x \in(-\infty, \infty)$, com $\rho=0$ se $x<x_{c}$, e $\rho=1$ se $x \geqslant x_{c}$, conforme ilustrado na Figura 11 (a) para $x \in[0,2]$ e $x_{c}=1$. Neste caso, tem-se uma fronteira bem definida, em $x_{c}=1$, que separa as fases sólida e vazia no domínio. Sobre o campo $\rho$, que representa o campo de projeto, aplica-se o filtro com decaimento linear, Equação (2.8), definido aqui com integrais em $x$, com $\vartheta \in[x-R, x+R]$, pois um domínio contínuo unidimensional está sendo considerado, tal que

$$
\tilde{\rho}=\frac{\int_{x-R}^{x+R} w\left(x_{i}\right) \rho_{i} d x_{i}}{\int_{x-R}^{x+R} w\left(x_{i}\right) d x_{i}} .
$$

Figura 11: Procedimento de filtragem e projeção utilizado: (a) campo de projeto; (b) campo filtrado; (c) campo projetado para $\delta \rightarrow \infty$; (d) campo projetado para $\delta=32$. Os campos projetados são avaliados para cinco valores distintos de $\eta$, da esquerda para direita: $0.1,0.25,0.5,0.75$ e 0.9 .

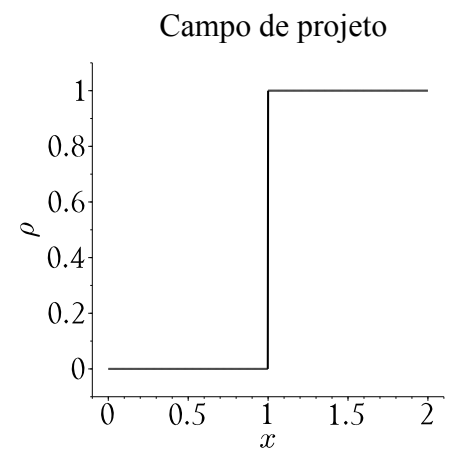

(a)

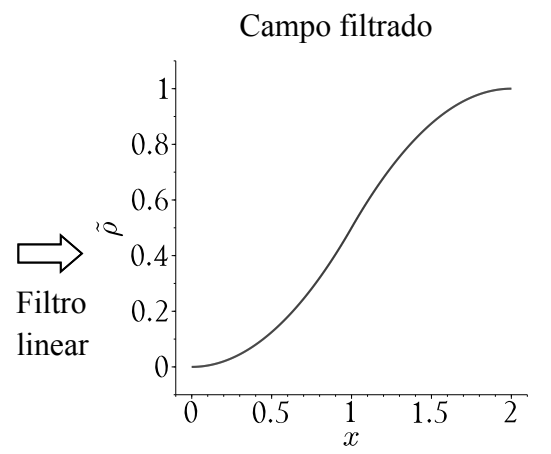

(b)

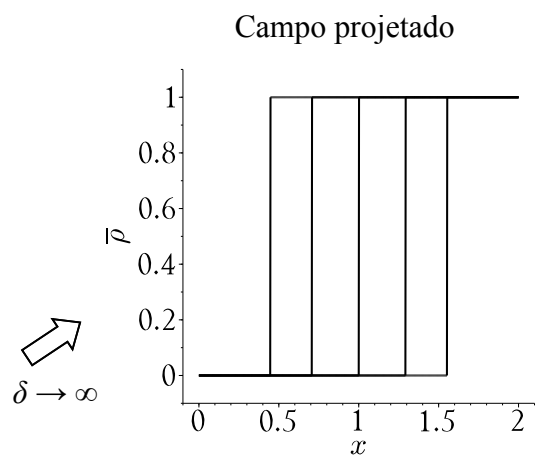

(c)

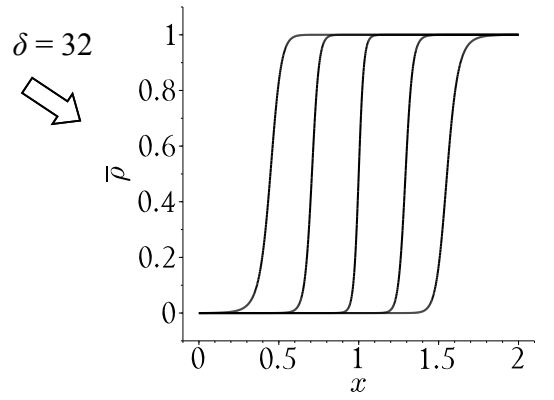

(d)

Fonte: adaptado de Silva, Beck e Sigmund (2019).

A função de decaimento linear, neste caso, é dada por $w\left(x_{i}\right)=R-\left|x_{i}-x\right|$. Substituindo $w\left(x_{i}\right)$ na Equação (5.21), e após algumas manipulações, obtém-se $\tilde{\rho}$, no intervalo $x \in\left[x_{c}-R, x_{c}+R\right]$, como

$$
\tilde{\rho}=\left\{\begin{array}{ll}
\frac{1}{2 R^{2}}\left(R+x-x_{c}\right)^{2} & \text { se } x \in\left[x_{c}-R, x_{c}\right) \\
\frac{1}{2 R^{2}}\left[2 R^{2}-\left(x-x_{c}-R\right)^{2}\right] & \text { se } x \in\left[x_{c}, x_{c}+R\right]
\end{array},\right.
$$


ilustrado na Figura 11 (b) para $R=1$ e $x_{c}=1$.

Quando a função de projeção, Equação (2.11), é aplicada sobre o campo filtrado, Equação (5.22), para $\delta \rightarrow \infty$, uma fronteira bem definida (0/1) é obtida, Figura 11 (c). Entretanto, quando um valor finito de $\delta$ é utilizado, material intermediário entre as fases sólida e vazia se torna evidente, Figura 11 (d).

Uma investigação numérica, feita por meio da substituição da Equação (5.22) na Equação (2.11), demonstrou que a maior derivada, dos campos projetados em relação a $x$, sempre ocorre para $\eta=0.5$, em $x=x_{c}$, derivadas as quais são ilustradas na Figura 12 para $x_{c}=R=1$. Como esta é a maior dentre todas as derivadas, este é o valor que deve ser limitado de forma a permitir uma transição de material intermediário entre as fases sólida e vazia da topologia de largura pelo menos igual ao tamanho de um elemento.

Figura 12: Derivadas dos campos projetados em relação à $x$, para $\delta=32($ Figura $11(\mathrm{~d}))$ e cinco valores diferentes de $\eta$.

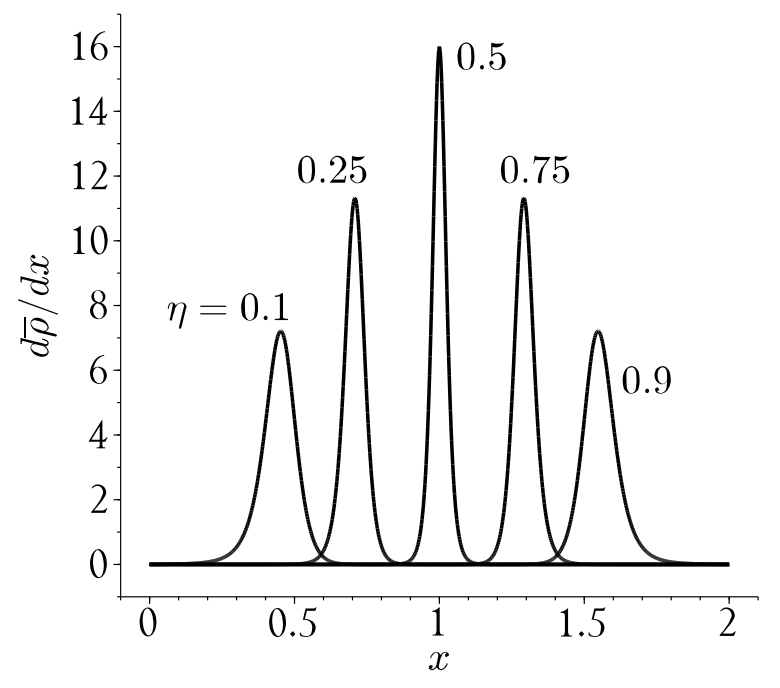

Fonte: Silva, Beck e Sigmund (2019).

Analiticamente, pode-se demonstrar que a inclinação da função que representa os campos projetados, avaliada em $\eta=0.5$ e $x=x_{c}$, é escrita como

$$
\tan \left(\alpha_{\max }\right)=\frac{0.5 \delta}{R \tanh (0.5 \delta)}
$$

Para valores elevados de $\delta$, uma relação mais simples é obtida

$$
\lim _{\delta \rightarrow \infty} \frac{\tan \left(\alpha_{\max }\right)}{\delta}=\frac{1}{2 R}
$$

cujo comportamento assintótico é ilustrado na Figura 13.

Baseando-se na Equação (5.24), a maior inclinação considerando um elevado valor de $\delta$ é dada por

$$
\tan \left(\alpha_{M a x}\right)=\frac{\delta}{2 R}
$$


Figura 13: Ilustração do comportamento assintótico da Equação (5.24), para $R=1$.

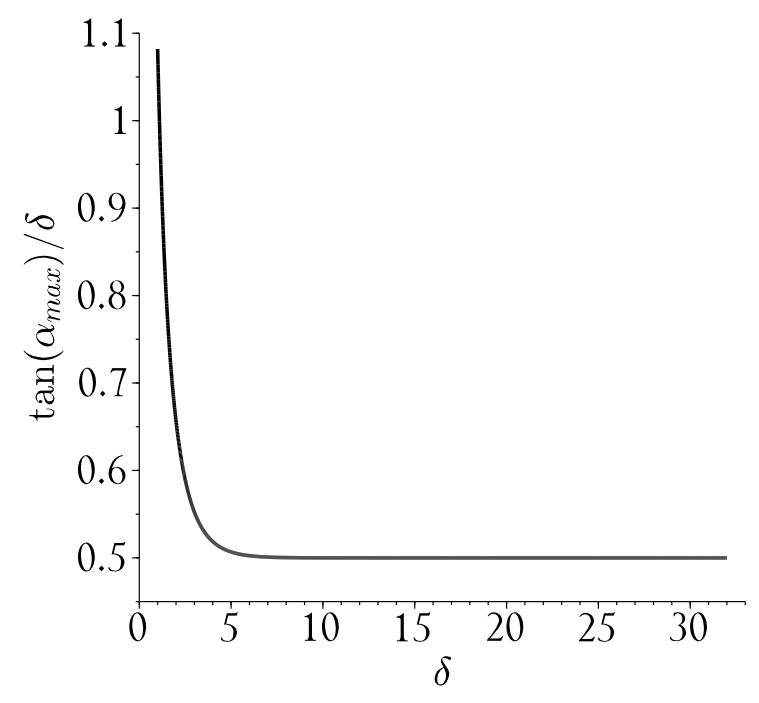

Fonte: adaptado de Silva, Beck e Sigmund (2019).

Agora, pode-se limitar a projeção considerando a distância entre dois picos de tensão consecutivos (de um elemento apenas $=l_{e}$ ), tal que

$$
\tan \left(\alpha_{l i m}\right)=\frac{1}{l_{e}}
$$

Finalmente, igualando $\tan \left(\alpha_{\text {Max }}\right)=\tan \left(\alpha_{\text {lim }}\right)$, define-se o valor de $\delta_{\text {lim }}$ como

$$
\delta_{\text {lim }}=\frac{2 R}{l_{e}} .
$$

O valor de $\delta_{l i m}$, definido na Equação (5.27), é empregado para permitir um contorno de material intermediário de transição com largura igual a pelo menos o tamanho de um elemento da malha. Entretanto, quando material intermediário é permitido na solução do problema baseado em tensão, as funções de interpolação de rigidez e tensão se tornam muito importantes, como demonstrado a seguir na subseção 5.2.3. Neste trabalho, escolhe-se trabalhar com a parametrização SIMP com $p=3$, como usualmente adotado na literatura, para interpolar a rigidez. Desta forma, deve-se escolher de forma adequada o parâmetro de relaxação $\varepsilon$ para interpolação das tensões, de forma a garantir tanto a acurácia no cálculo da tensão, como evitar fortes oscilações após variação de contorno uniforme.

\subsubsection{Escolhendo um parâmetro de interpolação de tensão adequado}

Nesta subseção, um estudo numérico é realizado: o problema de referência da chapa com furo circular é abordado novamente, considerando agora uma fina camada de material intermediário entre as fases sólida e vazia, ou seja, empregando um valor de $\delta$ finito, calculado com base no valor definido na Equação (5.27).

Um procedimento sistemático é empregado: 
1. Um campo com densidades $0 / 1$ é definido para $R_{h}=0.1$;

2. O filtro linear de densidades, Equação (2.8), é empregado sobre o campo 0/1, considerando $R=0.04$;

3. A função de projeção, Equação (2.11), é aplicada sobre as variáveis filtradas, considerando quatro valores de $\delta$, sendo eles: $2 \times \delta_{\text {lim }}, \delta_{\text {lim }}, \delta_{\text {lim }} / 2$ e $\delta_{\text {lim }} / 4$; para verificar o comportamento da tensão máxima considerando diferentes larguras de transição de material intermediário;

4. $\max \left(\sigma_{11}\right)$ é avaliado para cada campo projetado considerando os quatro valores de $\delta$ empregados.

Este procedimento é realizado para três malhas distintas: $200 \times 100,400 \times 200 \mathrm{e}$ $800 \times 400$; e cinco valores de $\varepsilon: 0.05,0.1,0.2,0.3,0.5$. Os campos projetados são obtidos para $\eta \in[0.25,0.75]$, de forma a obter variações de contorno de magnitude similar às análises previamente realizadas na subseção 5.2.1, onde $R_{h} \in[0.09,0.11]$. Os resultados são ilustrados na Figura 14.

Analisando a Figura 14, verifica-se que a escolha dos parâmetros $\delta$ e $\varepsilon$ não é óbvia. Entretanto, comparação com a solução de referência demonstra que estes parâmetros podem ser propriamente escolhidos, de forma a obter resposta de tensão consistente na transição entre as fases sólida e vazia.

O estudo numérico demonstra que não existe necessidade de garantir tensões fisicamente coerentes nas densidades intermediárias (ou seja, garantir $\varepsilon \rightarrow 0$ ), quando o objetivo é a avaliação das tensões da estrutura 0/1 subjacente. O estudo numérico também demonstra que, ao empregar a função de relaxação $\varepsilon$ na interpolação das tensões, boa concordância entre as tensões avaliadas com o modelo baseado em pixel e as tensões de referência pode ser obtida. Entretanto, escolha adequada do parâmetro $\varepsilon$ é necessária, tal como a consideração de uma largura adequada de transição de material intermediário entre as fases sólida e vazia.

Deve-se salientar que a abordagem SIMP é utilizada para interpolação da rigidez neste estudo, com o parâmetro $p=3$. Caso um parâmetro diferente seja empregado na interpolação da rigidez, ou uma função de interpolação diferente seja utilizada (por exemplo, a abordagem RAMP - Rational Approximation of Material Properties (STOLPE; SVANBERG, 2001)), o estudo numérico realizado nesta subseção não seria mais válido, e precisaria ser refeito, seguindo o mesmo procedimento apresentado no início desta subseção. A mesma conclusão se aplica se uma função de interpolação de tensão diferente for utilizada, como as funções $f_{\sigma}^{q p}$ e $f_{\sigma}^{\delta}$, apresentadas na subseção 4.1 .

Considerando as análises aqui realizadas, verifica-se que, em geral, se o valor de $\varepsilon$ é muito pequeno, as tensões máximas nas interfaces irregulares são superestimadas. 
Figura 14: Gráficos de tensão para diferentes valores de $\varepsilon$ e tamanhos de malha. Cada gráfico foi gerado para quatro valores diferentes de $\delta$ e comparado com a solução de referência $(\cong 3)$. O tamanho das linhas horizontais indica o tamanho dos elementos na malha.
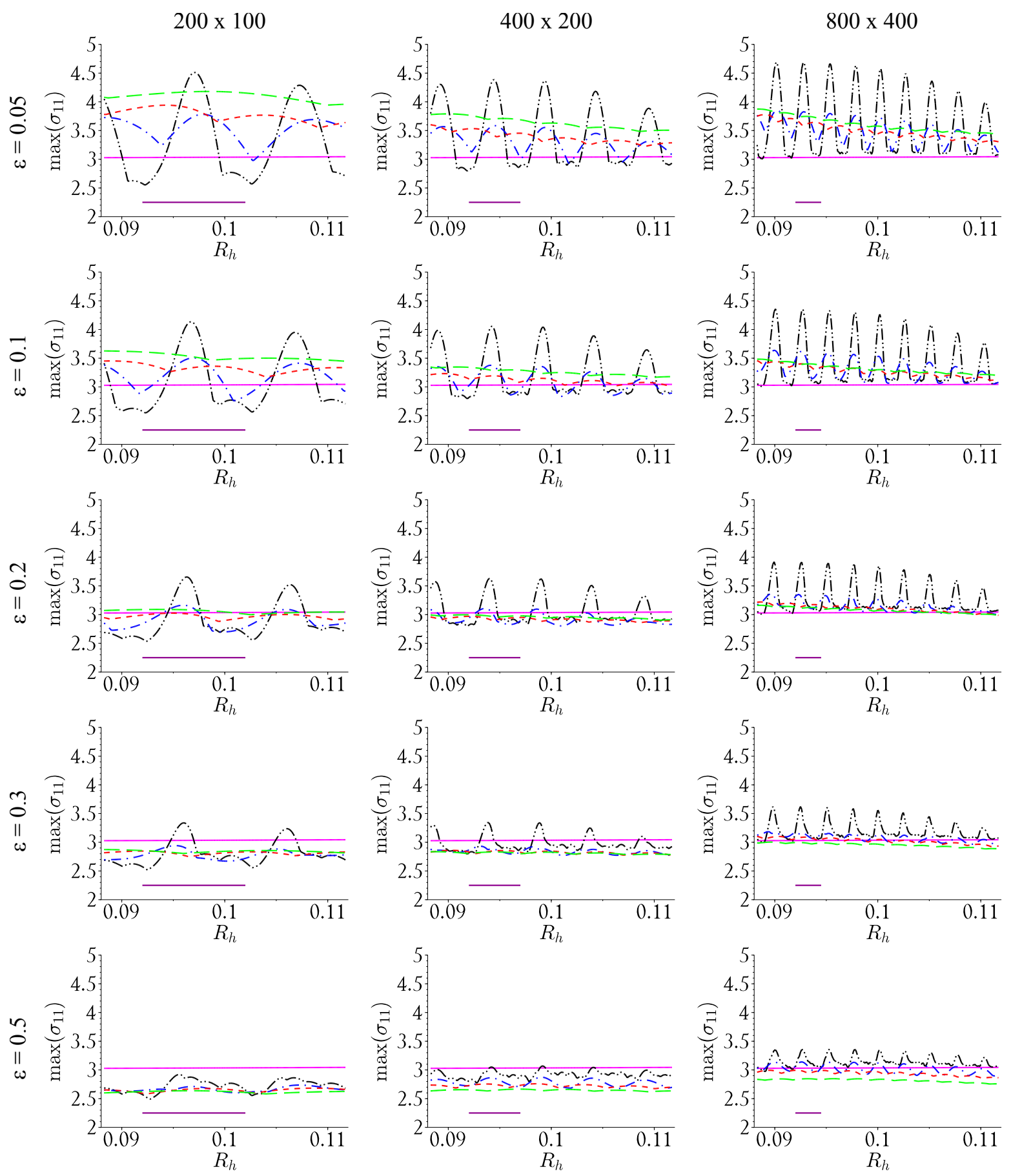

$$
\begin{array}{|l}
- \text { Referência } \\
\hdashline-\cdot-\delta=2 \times \delta_{\text {lim }} \\
-\cdot-\delta=\delta_{\text {lim }} \\
---\delta=\delta_{\text {lim }} / 2 \\
--\delta=\delta_{\text {lim }} / 4
\end{array}
$$

Fonte: adaptado de Silva, Beck e Sigmund (2019). 
Além disso, pequenos valores de $\varepsilon$ causam fortes oscilações de tensão após variação de contorno uniforme, o que pode afetar de forma negativa a convergência do procedimento de otimização e a qualidade dos resultados robustos. Por outro lado, se o valor de $\varepsilon$ é muito grande, as tensões são subestimadas. Para este exemplo, verifica-se que existe uma escolha adequada de $\varepsilon$, de forma a garantir acurácia no cálculo da tensão e pouca oscilação de tensão após variação de contorno uniforme, considerando $\delta \leqslant \delta_{\text {lim }}$.

Verifica-se, também, que quanto maior o valor de $\delta$, ou seja, quanto menor for a largura de transição de material intermediário entre as fases sólida e vazia, mais fortes são as oscilações de tensão devido a variação de contorno uniforme. Estas oscilações são ainda mais evidentes para pequenos valores de $\varepsilon$.

Na seção de resultados, os problemas abordados com a formulação proposta na Equação (5.20) são solucionados considerando $\varepsilon=0.2$. Baseando-se nos gráficos da Figura 14, verifica-se que esta escolha fornece uma boa relação entre acurácia e pouca oscilação de tensão dada variação de contorno uniforme, principalmente para $\delta \leqslant \delta_{\text {lim }} / 2$.

É importante enfatizar que todos os resultados provenientes deste estudo numérico, relativos ao problema da chapa com furo circular, foram obtidos com a utilização de elementos bilineares isoparamétricos de quatro nós quadrados. Além disso, as tensões foram calculadas no centroide de cada elemento, que é o ponto superconvergente para cálculo de tensão neste caso (BARLOW, 1976). Deve-se salientar que, quando elementos diferentes forem utilizados ou, alternativamente, quando as tensões forem calculadas em outros pontos que não os centroides (pontos de Gauss, por exemplo), diferentes gráficos de tensão podem ser obtidos. 



\section{SOLUÇÃO DO PROBLEMA DE OTIMIZAÇÃO}

Esta seção apresenta a abordagem que é utilizada para solucionar os problemas de otimização topológica na forma discretizada, definidos nas Equações (4.1), (5.2), (5.12), (5.18) e (5.20). A subseção 6.1 apresenta o método do Lagrangiano aumentado, baseando-se no trabalho de Birgin e Martínez (2014), utilizado para lidar com o elevado número de restrições de tensão presente nas formulações propostas. A subseção 6.2 apresenta um método de descida mais íngreme modificado, conforme proposto em Silva, Beck e Cardoso (2018), utilizado para solucionar os subproblemas de otimização resultantes do método do Lagrangiano aumentado empregado.

\subsection{Método do Lagrangiano aumentado}

A ideia essencial associada ao método do Lagrangiano aumentado consiste na transformação do problema de otimização restrito original em uma sequência de problemas de otimização irrestritos (ou menos restritos). A função objetivo dos subproblemas de otimização é o Lagrangiano aumentado, que consiste em uma soma ponderada entre a função objetivo do problema original e as restrições de projeto. Nesta formulação, cada restrição de projeto é associada a um multiplicador de Lagrange e a um parâmetro de penalização. O método do Lagrangiano aumentado consiste então em uma abordagem sequencial: após a solução do subproblema de otimização atual, os multiplicadores de Lagrange e parâmetros de penalização são atualizados; então, a solução do subproblema de otimização atual e os parâmetros atualizados são utilizados como estimativas iniciais para solucionar o próximo subproblema de otimização, e assim por diante, até a convergência; indicando que a solução do problema de otimização original foi obtida.

Neste trabalho, considera-se um único parâmetro de penalização para todas as restrições de projeto, por praticidade. Considerando apenas as restrições de tensão, escrevese o Lagrangiano aumentado do problema determinístico, Equação (4.1), como

$$
L(\overline{\boldsymbol{\rho}}, \boldsymbol{\mu}, r)=\frac{N_{e}}{\sum_{e=1}^{N_{e}} V_{e}} V(\overline{\boldsymbol{\rho}})+\frac{r}{2} \sum_{k=1}^{N_{k}}\left\langle\frac{\mu_{k}}{r}+\frac{\sigma_{e q}^{(k)}(\overline{\boldsymbol{\rho}})}{\sigma_{y}}-1\right\rangle^{2},
$$

onde $\boldsymbol{\mu} \in \mathbb{R}^{N_{k}}$ é um vetor que contém todos os multiplicadores de Lagrange do problema, $r$ é o parâmetro de penalização, $\mu_{k}$ é o multiplicador de Lagrange associado a $k$-ésima restrição de tensão e $\langle\cdot\rangle=\max (0, \cdot)$. A função objetivo é ponderada pela constante $\frac{N_{e}}{\sum_{e=1}^{N_{e}} V_{e}}$ para propósito de normalização.

Como as restrições laterais não são incluídas no Lagrangiano aumentado, nesta implementação, estas devem ser incluídas nos subproblemas de otimização, definidos como 


$$
\begin{array}{ll}
\underset{\boldsymbol{\rho}}{\operatorname{minimizar}} & L\left(\overline{\boldsymbol{\rho}}, \boldsymbol{\mu}^{(c)}, r^{(c)}\right) \\
\text { sujeito a } & 0 \leqslant \rho_{e} \leqslant 1 \quad e=1 . . N_{e} \\
& \mathbf{K}(\overline{\boldsymbol{\rho}}) \mathbf{U}(\overline{\boldsymbol{\rho}})=\mathbf{F}
\end{array}
$$

onde o índice ${ }^{(c)}$ é referente ao $c$-ésimo subproblema de otimização.

Após solucionar o c-ésimo subproblema, utiliza-se a solução $\left(\boldsymbol{\rho}^{(c)}\right)^{*}$ do subproblema atual, e as estimativas atuais dos multiplicadores de Lagrange, $\boldsymbol{\mu}^{(c)}$, e parâmetro de penalização, $r^{(c)}$, para obter as estimativas atualizadas destes parâmetros, utilizadas na solução do próximo subproblema de otimização, como

$$
\mu_{k}^{(c+1)}=\left\langle r^{(c)}\left(\frac{\sigma_{e q}^{(k)}\left(\left(\overline{\boldsymbol{\rho}}^{(c)}\right)^{*}\right)}{\sigma_{y}}-1\right)+\mu_{k}^{(c)}\right\rangle,
$$

e

$$
r^{(c+1)}=\left\{\begin{array}{ll}
\min \left(\gamma r^{(c)}, r_{\max }\right) & \text { se } \delta \sigma_{\max }^{(c)}>\omega \delta \sigma_{\max }^{(c-1)} \\
r^{(c)} & \text { caso contrário }
\end{array},\right.
$$

onde $\gamma>1$ e $\omega<1$ são parâmetros de atualização, $r_{\text {max }}$ é um valor limite de parâmetro de penalização e $\delta \sigma_{\max }=\left(\frac{\sigma_{e q}^{\max }}{\sigma_{y}}-1\right)$, onde $\sigma_{e q}^{\max }$ representa o máximo valor entre todas as tensões de von Mises calculadas. O parâmetro de penalização $r$ é multiplicado por $\gamma$ apenas se existir progresso razoável em relação a viabilidade da topologia otimizada, de forma a evitar um incremento desnecessário da não linearidade dos subproblemas de otimização.

Este procedimento é realizado até que os critérios de convergência sejam simultaneamente satisfeitos: 1) quando a máxima diferença entre as variáveis de projeto de dois subproblemas consecutivos for menor que $\left.t_{0} l_{\rho} ; 2\right)$ quando a viabilidade da estrutura otimizada estiver garantida, ou seja, $\frac{\sigma_{e q}^{\max }}{\sigma_{y}}-1<$ tol $_{\sigma}$.

O resultado deste procedimento é a solução do problema de otimização topológica para um dado valor de $\delta$, parâmetro utilizado nas funções de projeção, Equações (2.10) e (2.11). Nesta tese, no entanto, uma abordagem de continuação é empregada para atualizar o valor de $\delta$, de um valor pequeno até um valor máximo, visando a obtenção de uma convergência estável. Propõe-se, então, uma abordagem de continuação com base no método do Lagrangiano aumentado descrito nesta seção: o problema é inicialmente solucionado para $\delta \cong 0$, com o método do Lagrangiano aumentado; então, o valor de $\delta$ é incrementado e o próximo problema é solucionado, considerando a solução do problema atual, os multiplicadores de Lagrange atuais e o parâmetro de penalização atual como estimativas iniciais; e assim por diante; até que um valor máximo de $\delta$ seja obtido.

A Figura 15 mostra o fluxograma simplificado do algoritmo de solução proposto. Embora o algoritmo de solução tenha sido descrito utilizando o problema determinístico 
como base, Equação (4.1), este mesmo algoritmo é utilizado para solucionar todos os outros problemas de otimização topológica descritos nesta tese, Equações (5.2), (5.12), (5.18) e (5.20). A diferença está na escrita do Lagrangiano aumentado, Equação (6.1), conforme descrito a seguir nas subseções 6.1.1 e 6.1.2, e no cálculo das medidas de tensão empregadas em cada abordagem.

Figura 15: Fluxograma simplificado com a abordagem de solução proposta.

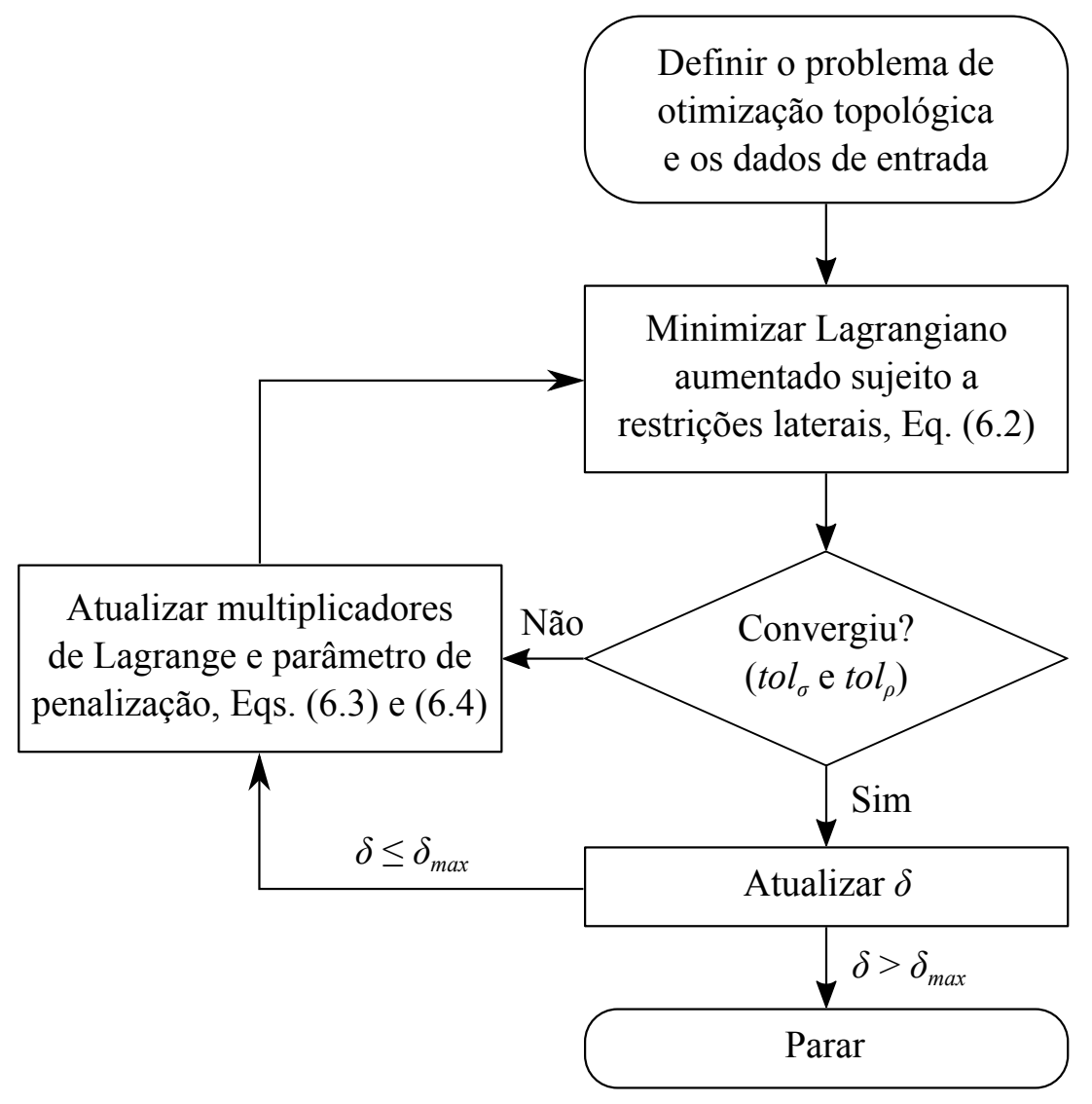

Fonte: produção do próprio autor.

\subsubsection{Problemas com incerteza nas forças}

Observando-se todas as formulações propostas onde incerteza nas forças é considerada, Equações (5.2), (5.12) e (5.18), verifica-se que estas são muito similares a formulação determinística, Equação (4.1). A principal diferença consiste na medida de tensão utilizada para formular o problema de otimização. Enquanto que na formulação determinística se utiliza a própria tensão equivalente de von Mises interpolada, $\sigma_{e q}^{(k)}(\overline{\boldsymbol{\rho}})$, nas formulações sob incerteza nas forças se utilizam medidas de tensão relativas a cada abordagem:

- $\hat{\sigma}_{e q}^{(k)}(\overline{\boldsymbol{\rho}}, \mathbf{Z})$ (robusta probabilística);

- $\sigma_{e q}^{(k)}\left(\overline{\boldsymbol{\rho}},\left(\mathbf{Y}^{(k)}\right)^{*}\right)$ (baseada em confiabilidade);

- $\sigma_{e q}^{(k)}\left(\overline{\boldsymbol{\rho}},\left(\mathbf{W}^{(k)}\right)^{*}\right)$ (robusta não probabilística). 
Obviamente, o cálculo de cada medida de tensão segue um procedimento diferente, dependendo da abordagem empregada: $\hat{\sigma}_{e q}^{(k)}(\overline{\boldsymbol{\rho}}, \mathbf{Z})$ é calculada como uma soma ponderada entre o valor esperado e o desvio padrão da tensão equivalente de von Mises, conforme descrito na subseção 5.1.1; $\sigma_{e q}^{(k)}\left(\overline{\boldsymbol{\rho}},\left(\mathbf{Y}^{(k)}\right)^{*}\right)$ é calculada com uma análise de confiabilidade inversa, Equação (5.13); e $\sigma_{e q}^{(k)}\left(\overline{\boldsymbol{\rho}},\left(\mathbf{W}^{(k)}\right)^{*}\right)$ é calculada com uma análise de pior cenário (anti-otimização), Equação (5.19). Entretanto, considerando-se que todas estas medidas de tensão podem ser representadas por uma função genérica $\sigma_{\text {gen }}^{(k)}(\overline{\boldsymbol{\rho}}, \mathbf{Q})$, onde $\mathbf{Q}$ representa as variáveis incertas do problema de otimização, escreve-se o Lagrangiano aumentado referente a cada uma destas formulações como:

$$
L(\overline{\boldsymbol{\rho}}, \mathbf{Q}, \boldsymbol{\mu}, r)=\frac{N_{e}}{\sum_{e=1}^{N_{e}} V_{e}} V_{p}(\overline{\boldsymbol{\rho}})+\frac{r}{2} \sum_{k=1}^{N_{k}}\left\langle\frac{\mu_{k}}{r}+\frac{\sigma_{g e n}^{(k)}(\overline{\boldsymbol{\rho}}, \mathbf{Q})}{\sigma_{y}}-1\right\rangle^{2} .
$$

Verifica-se que o Lagrangiano aumentado definido na Equação (6.5) é similar aquele definido na Equação (6.1). O procedimento de solução empregado é o mesmo, com a diferença no cálculo da medida de tensão $\sigma_{\text {gen }}^{(k)}(\overline{\boldsymbol{\rho}}, \mathbf{Q})$ a cada etapa do problema de otimização, que deve ser feita com base na formulação utilizada.

\subsubsection{Problema com incerteza de manufatura}

A formulação sob incerteza de manufatura, Equação (5.20), difere da formulação determinística, Equação (4.1), apenas no número de campos de densidades relativas sobre os quais as restrições de tensão são impostas. Neste caso, escreve-se o Lagrangiano aumentado como

$$
L\left(\overline{\boldsymbol{\rho}}^{(e)}, \overline{\boldsymbol{\rho}}^{(i)}, \overline{\boldsymbol{\rho}}^{(d)}, \boldsymbol{\mu}, r\right)=\frac{N_{e}}{\sum_{e=1}^{N_{e}} V_{e}} V\left(\overline{\boldsymbol{\rho}}^{(d)}\right)+\frac{r}{2} \sum_{k=1}^{N_{k}} \sum_{j \in\{e, i, d\}}\left\langle\frac{\mu_{k}^{(j)}}{r}+\frac{\sigma_{e q}^{(k)}\left(\overline{\boldsymbol{\rho}}^{(j)}\right)}{\sigma_{y}}-1\right\rangle^{2} .
$$

Na Equação (6.6), $\boldsymbol{\mu}$ representa uma matriz de multiplicadores de Lagrange de dimensão $N_{k} \times 3$, onde cada coluna representa um campo projetado $j \in\{e, i, d\}$. O procedimento de solução mostrado na Figura 15 também é utilizado para solucionar o problema com incerteza de manufatura.

\subsection{Solução dos subproblemas de otimização}

Conforme descrito na subseção 6.1, todos os problemas de otimização topológica, nesta tese, são solucionados com a abordagem proposta baseada no método do Lagrangiano aumentado. Uma das principais etapas do procedimento de solução, nesta abordagem, é a solução dos subproblemas de otimização, Equação (6.2). Neste trabalho, os subproblemas de otimização são solucionados com um método de descida mais íngreme modificado, conforme proposto em Silva, Beck e Cardoso (2018) e também descrito nesta subseção.

O método de descida mais íngreme modificado é descrito nas etapas a seguir: 
1. Iteração $b=1$ do subproblema $c$

2. Calcular a direção de máxima descida normalizada, como $\mathbf{D}=-\frac{\nabla_{\boldsymbol{\rho}} L\left(\boldsymbol{\rho}^{(b)}\right)}{\max \left|\nabla_{\boldsymbol{\rho}} L\left(\boldsymbol{\rho}^{(b)}\right)\right|}$

3. Obter o tamanho de passo $\Psi$ que garanta $L\left(\boldsymbol{\rho}^{(b+1)}\right)<L\left(\boldsymbol{\rho}^{(b)}\right)$, conforme a seguinte regra de atualização

$$
\rho_{e}^{(b+1)}= \begin{cases}\max \left(\rho_{e}^{\text {inf }}, 0\right) & \text { se } \rho_{e}^{(b)}+\Psi D_{e} \leqslant \max \left(\rho_{e}^{\text {inf }}, 0\right) \\ \min \left(\rho_{e}^{\text {sup }}, 1\right) & \text { se } \rho_{e}^{(b)}+\Psi D_{e} \geqslant \min \left(\rho_{e}^{\text {sup }}, 1\right) \\ \rho_{e}^{(b)}+\Psi D_{e} & \text { caso contrário }\end{cases}
$$

4. Se $\max \left|\boldsymbol{\rho}^{(b+1)}-\boldsymbol{\rho}^{(b)}\right|<t o l_{\text {sub }}$ ou $b=n i t_{\max }$ (número máximo de iterações)

Solução otimizada obtida, $\boldsymbol{\rho}^{(c)}=\boldsymbol{\rho}^{(b+1)}$

Parar procedimento

\section{Caso contrário}

Atualizar os limites móveis inferior $\rho_{e}^{\text {inf }}$ e superior $\rho_{e}^{\text {sup }}$, para $e=1 . . N_{e}$

Atualizar a iteração, $b=b+1$

Ir para o passo 2

O tamanho de passo $\Psi$ é obtido de forma inexata. Inicia-se com $\Psi^{(t e s t e)}=\min (1,2 \times$ $\left.\Psi^{(b-1)}\right)$, onde $\Psi^{(b-1)}$ é o tamanho de passo da última iteração, e realiza-se uma tentativa. Caso $L\left(\boldsymbol{\rho}^{(b+1)}\right) \geqslant L\left(\boldsymbol{\rho}^{(b)}\right)$, o tamanho de passo não é aceito, considera-se um novo tamanho de passo $\Psi^{(\text {teste })} \leftarrow \Psi^{(\text {teste })} / 2$, e efetua-se uma nova tentativa. Este procedimento é realizado até a obtenção de $L\left(\boldsymbol{\rho}^{(b+1)}\right)<L\left(\boldsymbol{\rho}^{(b)}\right)$. Em geral, o passo é aceito para $\Psi=1$, em uma única tentativa. Entretanto, quando estamos nos aproximando do mínimo local, algumas tentativas são necessárias e passos menores são considerados.

Existe um par de limites móveis associado com cada variável de projeto $\rho_{e}$, tal que $0 \leqslant \rho_{e}^{\text {inf }} \leqslant \rho_{e} \leqslant \rho_{e}^{\text {sup }} \leqslant 1$. Neste trabalho, utiliza-se uma simples abordagem heurística para atualizar os limites móveis a cada iteração, que consiste na redução dos limites móveis quando as variáveis de projeto oscilam em três iterações consecutivas, e no aumento dos mesmos caso contrário. Esta abordagem é frequentemente utilizada em algoritmos de programação linear sequencial e no método das assintotas móveis.

Se $\left(\rho_{e}^{(b+1)}-\rho_{e}^{(b)}\right) \times\left(\rho_{e}^{(b)}-\rho_{e}^{(b-1)}\right) \leqslant 0$, que indica oscilação, então

$$
\begin{aligned}
& \rho_{e}^{i n f}=\rho_{e}^{(b+1)}-k_{1}\left(\rho_{e}^{(b)}-\rho_{e}^{i n f}\right) \\
& \rho_{e}^{\text {sup }}=\rho_{e}^{(b+1)}+k_{1}\left(\rho_{e}^{\text {sup }}-\rho_{e}^{(b)}\right)
\end{aligned}
$$


Caso contrário

$$
\begin{aligned}
& \rho_{e}^{\text {inf }}=\rho_{e}^{(b+1)}-k_{2}\left(\rho_{e}^{(b)}-\rho_{e}^{\text {inf }}\right) \\
& \rho_{e}^{\text {sup }}=\rho_{e}^{(b+1)}+k_{2}\left(\rho_{e}^{\text {sup }}-\rho_{e}^{(b)}\right)
\end{aligned}
$$

$k_{1}$ e $k_{2}$ são constantes definidas pelo projetista, dentro dos intervalos $0<k_{1}<1$ e $k_{2}>1$. Neste trabalho, escolhe-se $k_{1}=0.7$ e $k_{2}=1.1$.

A atualização heurística dos limites móveis não é necessária, em teoria, para que o algoritmo utilizado obtenha um ponto de mínimo local. Esta atualização é unicamente utilizada para evitar oscilações excessivas nas variáveis de projeto que podem ocorrer dada a natureza não linear dos problemas baseados em tensão em estudo. Na prática, bons resultados são obtidos com esta abordagem, como mostrado a seguir na seção de resultados.

O método utilizado na solução dos subproblemas de otimização consiste em uma abordagem baseada em derivada. A derivada do Lagrangiano aumentado em relação a uma variável de projeto $\rho_{m}$, que representa a $m$-ésima componente de $\nabla_{\rho} L$, é obtida com uma análise de sensibilidade analítica com a abordagem adjunta. As análises de sensibilidade são realizadas especificamente para cada formulação e descritas nos trabalhos resultantes desta tese: em Silva, Beck e Cardoso (2018), para a formulação robusta probabilística; em Silva e Beck (2018) para a formulação baseada em confiabilidade; em Silva, Cardoso e Beck (2018) para a formulação robusta não probabilística; e em Silva, Beck e Sigmund (2019) para a abordagem robusta não probabilística sob incerteza de manufatura. 


\section{RESULTADOS NUMÉRICOS E DISCUSSÕES}

Esta seção apresenta os resultados numéricos obtidos a partir das formulações propostas na seção 5, utilizando-se o procedimento de solução proposto na seção 6 . A subseção 7.1 mostra os resultados obtidos com as diferentes formulações que lidam com incertezas nas forças. A subseção 7.2 mostra os resultados obtidos com a formulação tolerante a incerteza uniforme de manufatura. Considera-se estado plano de tensão em todos os exemplos apresentados. Alguns resultados apresentados nas subseções a seguir já estão publicados nos artigos desenvolvidos como fruto desta tese (SILVA; BECK; CARDOSO, 2018; SILVA; BECK, 2018; SILVA; CARDOSO; BECK, 2018; SILVA; BECK; SIGMUND, 2019).

\subsection{Problema com restrição de tensão sob incerteza nas forças}

Esta subseção é dividida em três partes:

- Subseção 7.1.1, que apresenta um estudo de dependência da malha realizado sobre problemas determinísticos;

- Subseção 7.1.2, que apresenta resultados de problemas sob incerteza na intensidade das forças aplicadas;

- Subseção 7.1.3, que apresenta resultados de problemas sob incerteza na direção das forças aplicadas.

Resultados adicionais são mostrados em Silva, Beck e Cardoso (2018), Silva e Beck (2018), Silva, Cardoso e Beck (2018).

7.1.1 Problema determinístico: estudo de dependência da malha

Nesta subseção, duas variantes do problema clássico em forma de L, Figura 16, são utilizadas em um estudo de dependência da malha, realizado para verificação do procedimento de solução proposto. Problemas em forma de L são frequentemente utilizados, na literatura, como problemas de benchmark, com o intuito de testar novos algoritmos e formulações de otimização topológica baseada em tensão (LE et al., 2010; PICELLI et al., 2018). Estes problemas são bastante interessantes, do ponto de vista de projeto baseado em tensão, pois o domínio em forma de L possui um canto de singularidade de tensão, que deve ser evitado pelo algoritmo de forma a evitar um canto vivo na solução otimizada.

Dados de entrada associados ao método de solução: $r^{(1)}=0.01, r_{\max }=10000$, $\gamma=10$ e $\omega=0.8$, associados ao método do Lagrangiano aumentado; tol $_{\rho}=0.1 \mathrm{e}$ 
Figura 16: Domínios em forma de L considerados, com dimensões e condições de contorno.
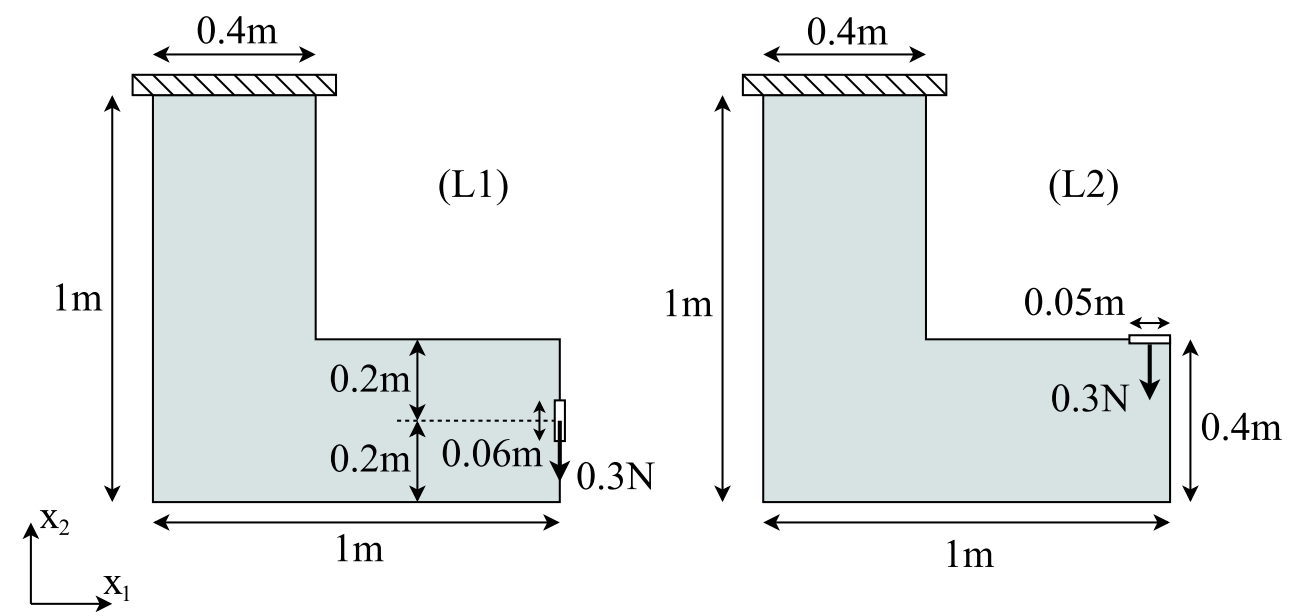

Fonte: adaptado de Silva, Cardoso e Beck (2018).

$t o l_{\sigma}=0.01$ como critério de convergência do problema externo de otimização; tol $_{\text {sub }}=0.01$ ou nit $_{\text {max }}=50$ como critério de convergência dos subproblemas de otimização. Escolhem-se $k_{1}=0.7$ e $k_{2}=1.1$ de forma a atualizar os limites móveis a cada iteração nos subproblemas de otimização. Utiliza-se $\delta^{(1)}=0$ e $\delta^{(d+1)} \leftarrow \min \left[\left(\delta^{(d)}+5\right), 100\right]$ como abordagem de continuação do filtro de densidade com projeção de dilatação, Equação (2.10), onde o índice $d$ indica a $d$-ésima iteração que satisfaz o critério de convergência do problema externo $\left(t o l_{\rho}\right.$ e $\left.t o l_{\sigma}\right)$. No início de cada subproblema de otimização, os limites móveis assumem os seus valores máximos $( \pm 0.1)$, se $\delta=0$, ou mínimos $( \pm 0.02)$, se $\delta>0$. Limites móveis mais estreitos são preferíveis no início de cada subproblema de otimização quando o passo não linear de projeção é aplicado, de forma a garantir maior estabilidade durante a otimização.

Dados de entrada associados às funções de interpolação: $p=3$ para interpolação da rigidez (SIMP); $\delta_{\sigma}=3$ para interpolação da tensão; $\delta_{v}=5$ para interpolação do volume.

Propriedades do material e características geométricas: módulo de elasticidade de $1 \mathrm{MPa}$, espessura de $1 \mathrm{~mm}$, coeficiente de Poisson de 0.3, tensão limite de $16 \mathrm{kPa}$ e raio de filtragem de $R=0.02 \mathrm{~m}$.

Dados adicionais: estimativa inicial das variáveis de projeto $\boldsymbol{\rho}^{(1)}=\mathbf{1}$. Os domínios de projeto são discretizados com elementos bilineares isoparamétricos de quatro nós quadrados, e as tensões são calculadas no centroide de cada elemento finito. As topologias são ilustradas com uma escala de cinza tradicional, onde branco representa a fase vazia $(\bar{\rho}=0)$ e preto representa a fase sólida $(\bar{\rho}=1)$, Figura 17. As tensões de von Mises são ilustradas com uma escala de cores: vermelho representa a tensão máxima normalizada $(\cong 1)$ e azul representa a tensão mínima normalizada $(\cong 0)$, Figura 18. As forças são distribuídas em $0.06 \mathrm{~m}$, problema (L1), e 0.05m, problema (L2), para evitar concentrações de tensão. 
Figura 17: Escala de cores utilizada para ilustração de topologias. Branco representa a fase vazia $(\bar{\rho}=0)$ e preto a fase sólida $(\bar{\rho}=1)$ da topologia.

Fonte: produção do próprio autor.

Figura 18: Escala de cores utilizada para ilustração de tensões de von Mises e índices de confiabilidade pós-processados. Nos gráficos de tensão, vermelho representa

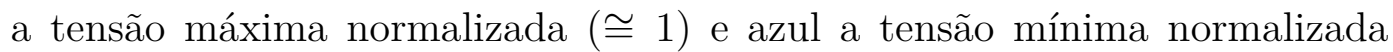
$(\cong 0)$. Nos gráficos de índices de confiabilidade, vermelho representa o índice de confiabilidade mínimo e azul o máximo.

Fonte: produção do próprio autor.

Os problemas (L1) e (L2) são solucionados considerando quatro discretizações distintas: $N_{e}=57600(300 \times 300) ; 102400(400 \times 400) ; 160000(500 \times 500)$; e 230400 $(600 \times 600)$ elementos. As topologias otimizadas e respectivas tensões equivalentes de von Mises são ilustradas nas Figuras 19 e 20, para os problemas (L1) e (L2), respectivamente.

Figura 19: Topologias otimizadas e respectivas tensões equivalentes de von Mises para diferentes discretizações. Da esquerda para direita: $N_{e}=57600(300 \times 300)$; $N_{e}=102400(400 \times 400) ; N_{e}=160000(500 \times 500) ; N_{e}=230400(600 \times 600)$. Problema (L1) determinístico.
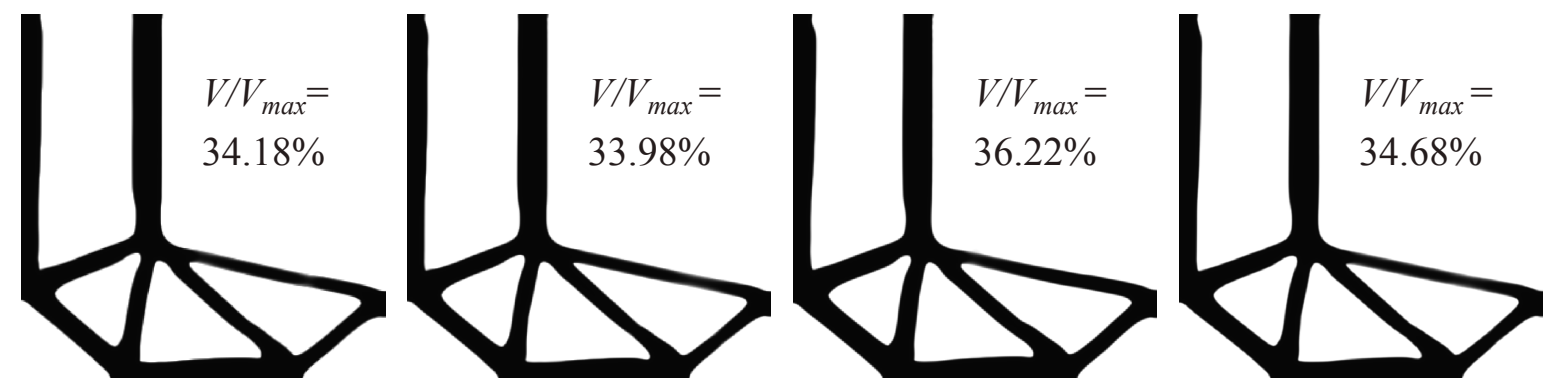

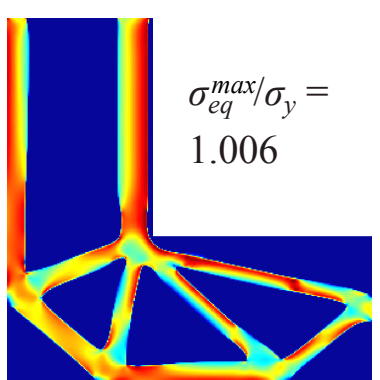

$N_{e}=57600$

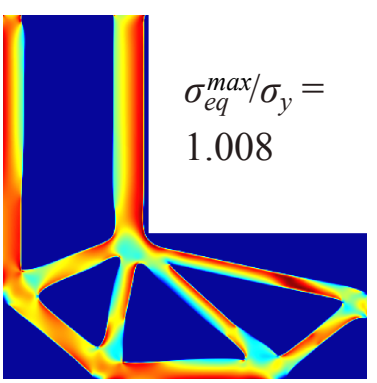

$N_{e}=102400$

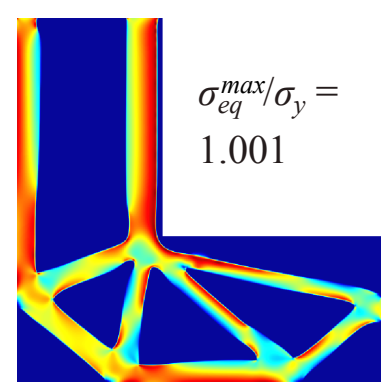

$N_{e}=160000$

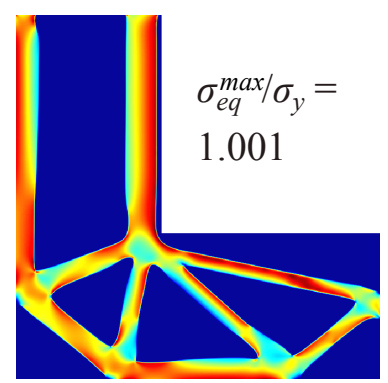

$N_{e}=230400$

Fonte: produção do próprio autor.

As Figuras 19 e 20 mostram que as topologias obtidas são independentes da malha, no sentido em que malhas mais refinadas fornecem topologias idênticas, possuindo apenas diferenças nas suas formas/contornos. Além disso, verifica-se que as topologias obtidas evitam o canto de singularidade de tensão, e que as tensões nestas regiões satisfazem o critério de falha em tensão considerado. 
Figura 20: Topologias otimizadas e respectivas tensões equivalentes de von Mises para diferentes discretizações. Da esquerda para direita: $N_{e}=57600(300 \times 300)$; $N_{e}=102400(400 \times 400) ; N_{e}=160000(500 \times 500) ; N_{e}=230400(600 \times 600)$. Problema (L2) determinístico.
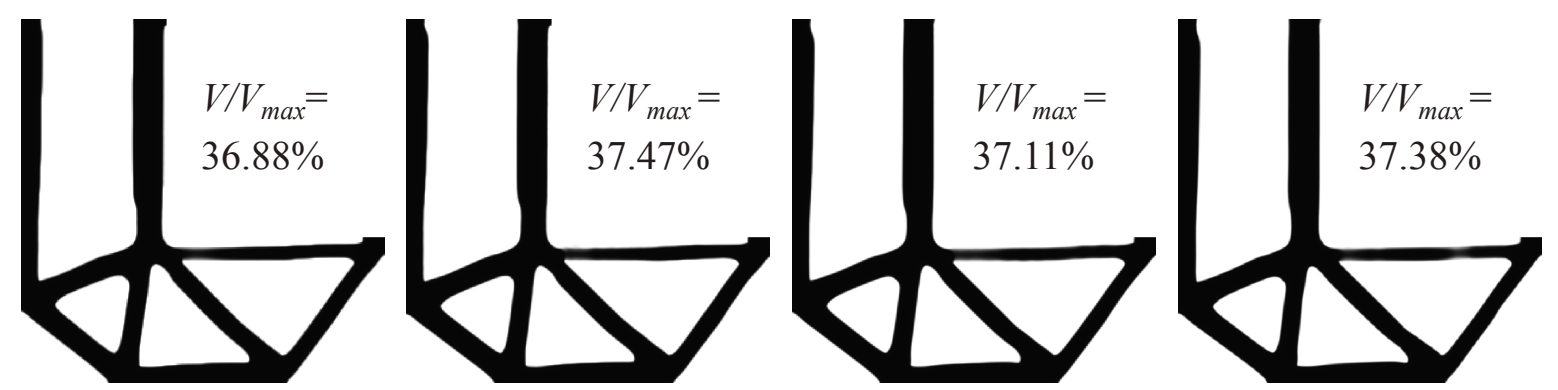

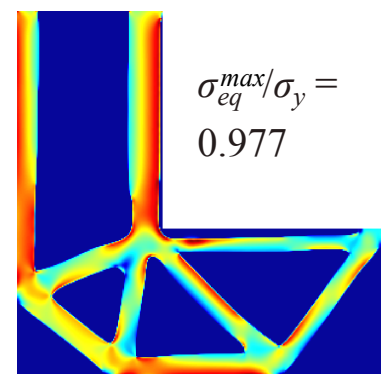

$N_{e}=57600$

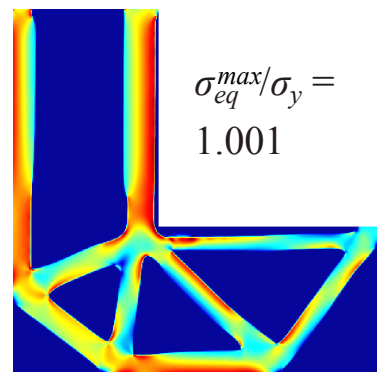

$N_{e}=102400$

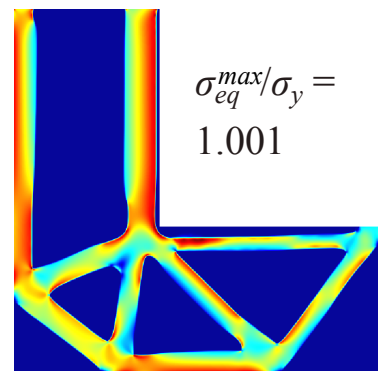

$N_{e}=160000$

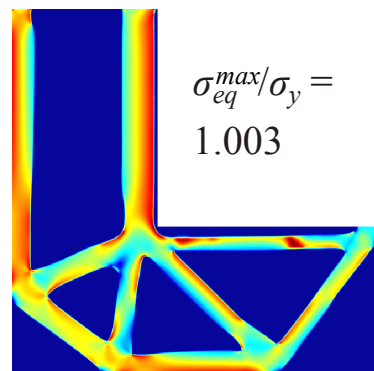

$N_{e}=230400$

Fonte: produção do próprio autor.

A Tabela 1 mostra o número de iterações necessárias até a convergência para cada problema de otimização. O número de iterações varia com o tipo de problema e número de elementos utilizados na discretização, sendo maior para o problema (L1) e, em geral, para malhas mais refinadas. Em média, o algoritmo precisa de aproximadamente 1800 iterações para obter a topologia otimizada, dados os critérios de convergência estabelecidos a priori.

Tabela 1: Número de iterações até a convergência, problemas determinísticos (L1) e (L2).

\begin{tabular}{lcc}
\hline Número de elementos & Problema (L1) & Problema (L2) \\
\hline$N_{e}=57600$ & 1675 & 1308 \\
$N_{e}=102400$ & 2274 & 1517 \\
$N_{e}=160000$ & 1749 & 1645 \\
$N_{e}=230400$ & 2292 & 1870 \\
\hline
\end{tabular}

Fonte: produção do próprio autor.

O número total de iterações é bastante dependente do número máximo de iterações de cada subproblema de otimização, escolhido como nit $_{\max }=50$. Pode-se reduzir o número total de iterações reduzindo $n i t_{\max }$, como realizado na subseção 7.2 , onde os problemas são solucionados para $n i t_{\max }=20$. Nesta subseção, escolhe-se $n i t_{\max }=50 \mathrm{com}$ o intuito de se realizar uma comparação justa entre as abordagens determinística e sob incerteza. Problemas baseados em tensão sob incerteza nas forças aplicadas são extremamente não lineares, o que exige um maior número de iterações na solução de cada subproblema de otimização para garantir a estabilidade do algoritmo. Obviamente, pode-se escolher um 
algoritmo diferente daquele empregado nesta tese para a solução dos subproblemas de otimização, subseção 6.2, com o intuito de melhorar a convergência e reduzir o número total de iterações.

A Figura 21 mostra o histórico de convergência da tensão de von Mises máxima normalizada, $\sigma_{e q}^{\max } / \sigma_{y}$, e da fração de volume da topologia, $V / V_{\max }$, considerando os resultados obtidos para as malhas mais refinadas $\left(N_{e}=230400\right)$ referentes aos problemas (L1) e (L2).

Figura 21: Históricos de convergência associados aos resultados obtidos para as malhas mais refinadas $\left(N_{e}=230400\right)$. Problemas: (L1), esquerda; e (L2), direita.
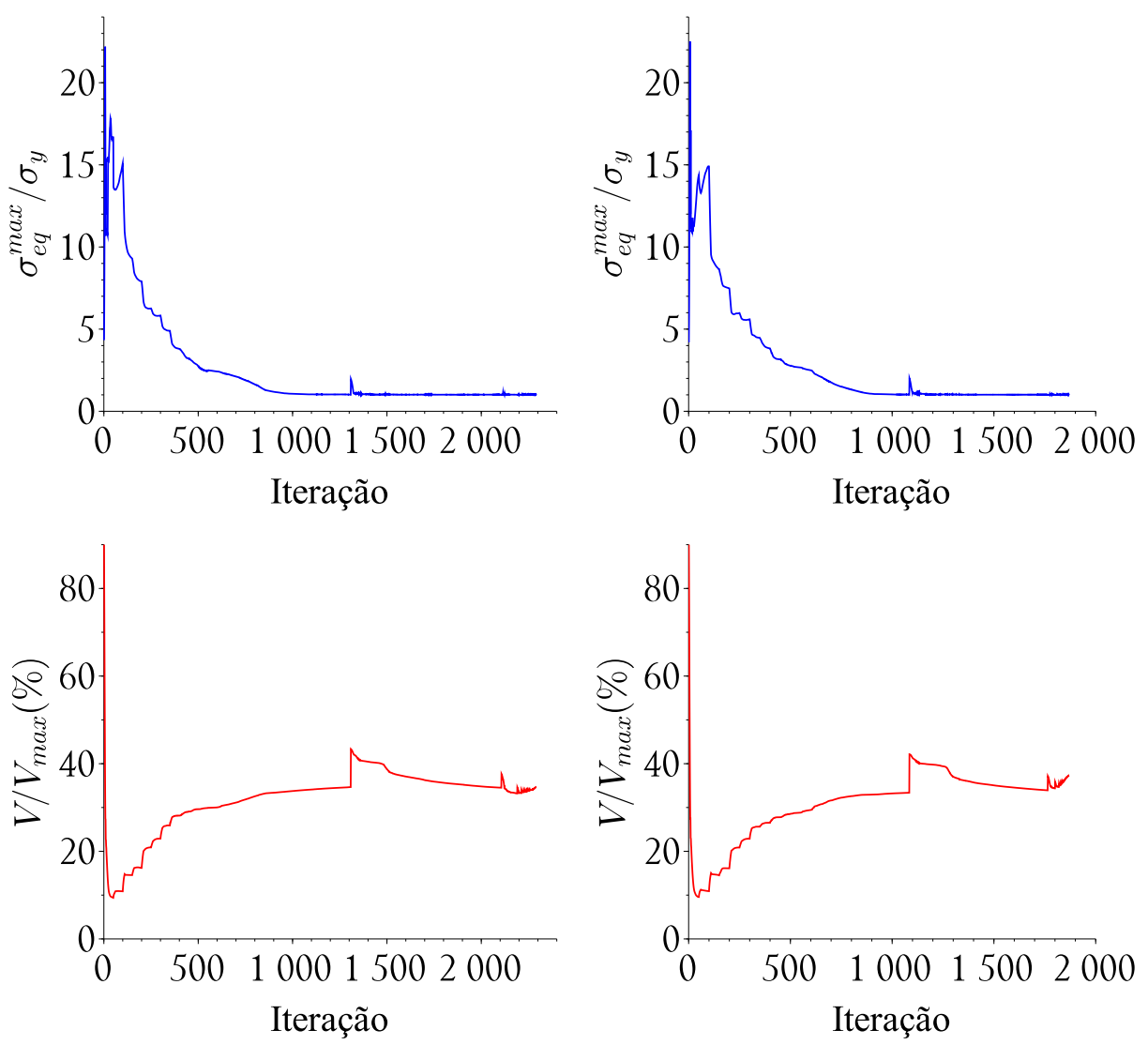

Fonte: produção do próprio autor.

Pode-se verificar, em um primeiro momento e apesar do diferente número de iterações, que os históricos de convergência de ambos os problemas são parecidos, no sentido em que os gráficos apresentam o mesmo comportamento. Como um parâmetro de penalização pequeno, $r^{(1)}$, é empregado no início do procedimento de otimização, as restrições de tensão têm uma pequena influência sobre o Lagrangiano aumentado, e consequentemente, o procedimento é dominado pela minimização do volume. Este fenômeno é claramente observado nos gráficos de volume, onde os volumes estruturais atingem uma fração muito pequena ( $\cong 10 \%)$ em poucas iterações.

Nas primeiras iterações, pode-se observar grandes valores de tensão máxima (até 
22 vezes o valor da tensão limite), tal como um comportamento oscilatório destes valores, o que também é justificado pela pequena influência das restrições de tensão sobre a função objetivo nestas iterações iniciais. Nos estágios iniciais do procedimento de otimização, após algumas atualizações dos multiplicadores de Lagrange e parâmetro de penalização, o peso das restrições de tensão (na função objetivo) aumenta, afetando diretamente o procedimento numérico, que começa a ser dominado pelas tensões. O efeito causado pelo aumento da influência das restrições de tensão sobre o procedimento de otimização é verificado em ambos os gráficos: a tensão de von Mises máxima começa a diminuir, enquanto que a fração de volume começa a aumentar.

Nos históricos de tensão máxima, pode-se verificar que, após a fase de estabilização inicial, a tensão de von Mises máxima começa a decrescer de forma suave e estável, sem muitas oscilações. Após a tensão máxima normalizada atingir um valor próximo de 1 , pode-se verificar somente um pico de tensão para cada gráfico de convergência: próximo a 1300 iterações para o problema (L1) e próximo a 1100 iterações para o problema (L2). Os picos de tensão indicam que o primeiro estágio do procedimento de otimização está completo, ou seja, a solução do problema de otimização para $\delta=0$ (filtro linear) foi obtida. A partir deste ponto, a aproximação suave para a função Heaviside é empregada para reduzir a quantidade de material intermediário entre as fases sólida e vazia da topologia. Como a função de projeção empregada é um operador de dilatação (SIGMUND, 2007), na primeira vez que a aproximação Heaviside é aplicada, a topologia dilata e o seu contorno encosta no canto reentrante do domínio de projeto, ocasionando um pequeno pico de tensão. Como pode ser visto nos históricos de tensão máxima, o algoritmo supera este problema em poucas iterações, e a tensão máxima normalizada decresce novamente, até atingir um valor próximo de 1 . O efeito de dilatação também é observado nos gráficos de volume, onde o volume estrutural aumenta no momento em que os picos de tensão são observados. Após esta ocorrência, o procedimento continua até que um valor máximo de $\delta$ seja obtido, e que os critérios de convergência sejam satisfeitos.

\subsubsection{Problema sob incerteza na intensidade das forças}

Esta subseção aborda o problema baseado em tensão sob incerteza na intensidade das forças aplicadas. Dois problemas são abordados: (a) problema de domínio retangular sujeito a duas forças incertas; (b) problema (L1) sujeito a uma força incerta; Figura 22. Todas as abordagens propostas na subseção 5.1 são utilizadas para solucionar ambos os problemas. Os resultados obtidos são comparados com os resultados determinísticos, onde consideram-se as topologias obtidas para os valores médios das forças aplicadas. Os dados de entrada para o algoritmo de otimização e as propriedades do material são os mesmos considerados na subseção 7.1.1, a menos que especificado de outra forma. 
Figura 22: Problemas considerados na subseção 7.1.2: (a) problema de domínio retangular sob duas forças incertas; (b) problema (L1) sob uma força incerta e uma força determinística; com dimensões e condições de contorno.

(a)

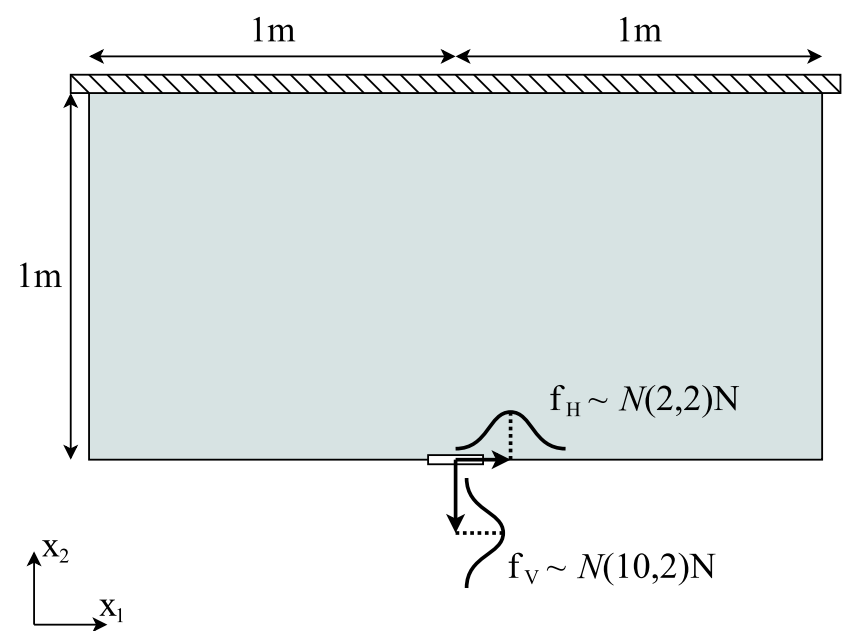

(b)

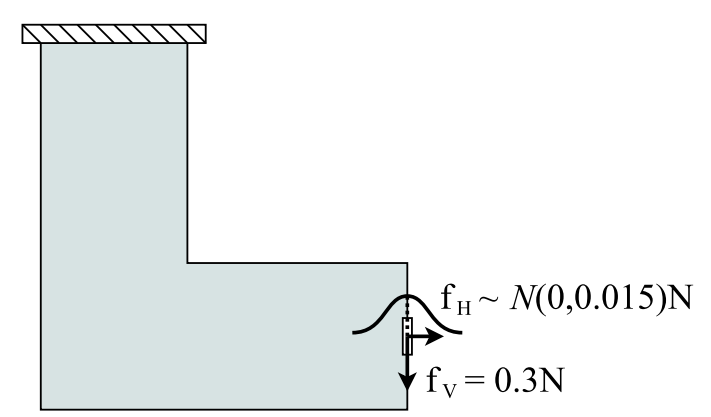

Fonte: produção do próprio autor.

\subsubsection{Problema de domínio retangular sob duas forças incertas}

O problema de domínio retangular, Figura 22 (a), é discretizado com uma malha estruturada regular de 80000 elementos. Dados de entrada: raio do filtro $R=0.04 \mathrm{~m}$ e tensão de escoamento $\sigma_{y}=100 \mathrm{kPa}$. Duas forças são aplicadas sobre o problema, de intensidade incerta e direção determinística, uma horizontal e uma vertical. As forças possuem intensidade Gaussiana $f \sim N(\mathrm{E}[f]$, Std $[f])$. A força horizontal possui média de $2 \mathrm{~N}$ e desvio padrão de $2 \mathrm{~N}, f_{H} \sim N(2,2) \mathrm{N}$, e a força vertical possui média de $10 \mathrm{~N}$ e desvio padrão de $2 \mathrm{~N}, f_{V} \sim N(10,2) \mathrm{N}$. As variáveis $f_{H}$ e $f_{V}$ são não correlacionadas. As forças aplicadas são distribuídas sobre um comprimento de $0.2 \mathrm{~m}$, para evitar concentrações de tensão.

Obviamente, como forças Gaussianas são consideradas, as abordagens mais adequadas que podem ser empregadas para solucionar o problema de otimização são as abordagens probabilísticas. Entretanto, de forma a comparar as abordagens determinística e não determinísticas, três problemas distintos são formulados, baseados em como as forças aplicadas são tratadas:

- Problema determinístico, com $f_{V}=10 \mathrm{~N}$ e $f_{H}=2 \mathrm{~N}$ (avaliam-se as forças nos seus valores médios);

- Problema probabilístico, considerando as forças Gaussianas conforme definidas no parágrafo anterior. Dados adicionais: $\alpha=2$, abordagem robusta probabilística, Equação (5.2); $\beta_{T}=2$, abordagem baseada em confiabilidade, Equação (5.12);

- Problema não probabilístico, onde as intensidades incertas são limitadas como: 
$f_{V} \in[6,14] \mathrm{N}$ e $f_{H} \in[-2,6] \mathrm{N}$. Os limites são definidos considerando $\mathrm{E}[f] \pm 2 \times \operatorname{Std}[f]$ (uma vez que se considera $\alpha=\beta_{T}=2$ no problema probabilístico).

Considerando especificamente o problema robusto não probabilístico, solucionam-se os problemas de anti-otimização com a abordagem de duas etapas descrita na subseção 5.1.3. Para cada ponto de cálculo de tensão: 1) avalia-se a tensão equivalente de von Mises para 9 pontos (combinações de $\mathbf{f}_{H}^{\text {grid }}=[-2,2,6]^{T} \mathrm{~N}$ e $\mathbf{f}_{V}^{\text {grid }}=[6,10,14]^{T} \mathrm{~N}$ ); 2) partindo do ponto que fornece a tensão de von Mises máxima, emprega-se o minimizador local para a obtenção da solução do problema de anti-otimização.

Os resultados obtidos são pós-processados com SMC, para $f_{H} \sim N(2,2) \mathrm{N}$ e $f_{V} \sim N(10,2) \mathrm{N}$. Os índices de confiabilidade, $\beta^{(k)}=-\Phi^{-1}\left(P_{f}^{(k)}\right)$, são calculados para cada ponto de cálculo de tensão $k$, onde $P_{f}^{(k)}$ é obtida dividindo o número de falhas em $k$ pelo número total de realizações. Utilizam-se $1 \times 10^{6}$ realizações. Um índice de confiabilidade máximo de $\beta_{\max }=-\Phi^{-1}\left(1 \times 10^{-6}\right) \cong 4.75$ é utilizado para ilustrar os índices de confiabilidade pós-processados.

A Figura 23 mostra as topologias, tensões de von Mises e índices de confiabilidade pós-processados. As tensões de von Mises ilustradas são as próprias medidas de tensão definidas para cada problema: $\sigma_{e q}^{(k)}\left(\overline{\boldsymbol{\rho}}^{*}\right)$ (determinístico); $\hat{\sigma}_{e q}^{(k)}\left(\overline{\boldsymbol{\rho}}^{*}, \mathbf{Z}\right)$ (robusto probabilístico); $\sigma_{e q}^{(k)}\left(\overline{\boldsymbol{\rho}}^{*},\left(\mathbf{Y}^{(k)}\right)^{*}\right)$ (baseado em confiabilidade); $\sigma_{e q}^{(k)}\left(\overline{\boldsymbol{\rho}}^{*},\left(\mathbf{W}^{(k)}\right)^{*}\right)$ (robusto não probabilístico).

Analisando a Figura 23, observa-se que as topologias obtidas com as abordagens não determinísticas são formadas por dois membros, em vez de um único membro como a topologia determinística. Entretanto, embora as topologias não determinísticas sejam idênticas, diferentes formas e volumes estruturais são observados. Os índices de confiabilidade pós-processados demonstram que as estruturas não determinísticas são mais confiáveis que a determinística, pois os seus índices de confiabilidade mínimos resultaram próximos do índice de confiabilidade alvo do problema baseado em confiabilidade, ou seja, $\beta_{\min } \cong \beta_{T}$. As probabilidades de falha máximas (associadas aos índices de confiabilidade mínimos) são mostradas na Tabela 2.

Tabela 2: Volumes estruturais, índices de confiabilidade mínimos, probabilidades de falha máximas e número de iterações até a convergência. Problema de domínio retangular.

\begin{tabular}{lcccc}
\hline Problema & $V / V_{\max }$ & $\beta_{\min }$ & $P_{f}^{\max }$ & Iterações \\
\hline Determinístico & $6.38 \%$ & -1.448 & $92.62 \%$ & 942 \\
Robusto prob. & $12.94 \%$ & 2.026 & $2.14 \%$ & 1334 \\
Baseado conf. & $13.03 \%$ & 2.027 & $2.13 \%$ & 1250 \\
Robusto não prob. & $14.63 \%$ & 2.182 & $1.46 \%$ & 1359 \\
\hline
\end{tabular}

Fonte: produção do próprio autor. 
Figura 23: Resultados do problema de domínio retangular: topologias (esquerda); tensões de von Mises (centro); índices de confiabilidade pós-processados com SMC (direita). As tensões ilustradas são as próprias medidas de tensão adotadas em cada problema. Os volumes estruturais, tensões máximas e índices de confiabilidade mínimos são mostrados abaixo de cada figura.

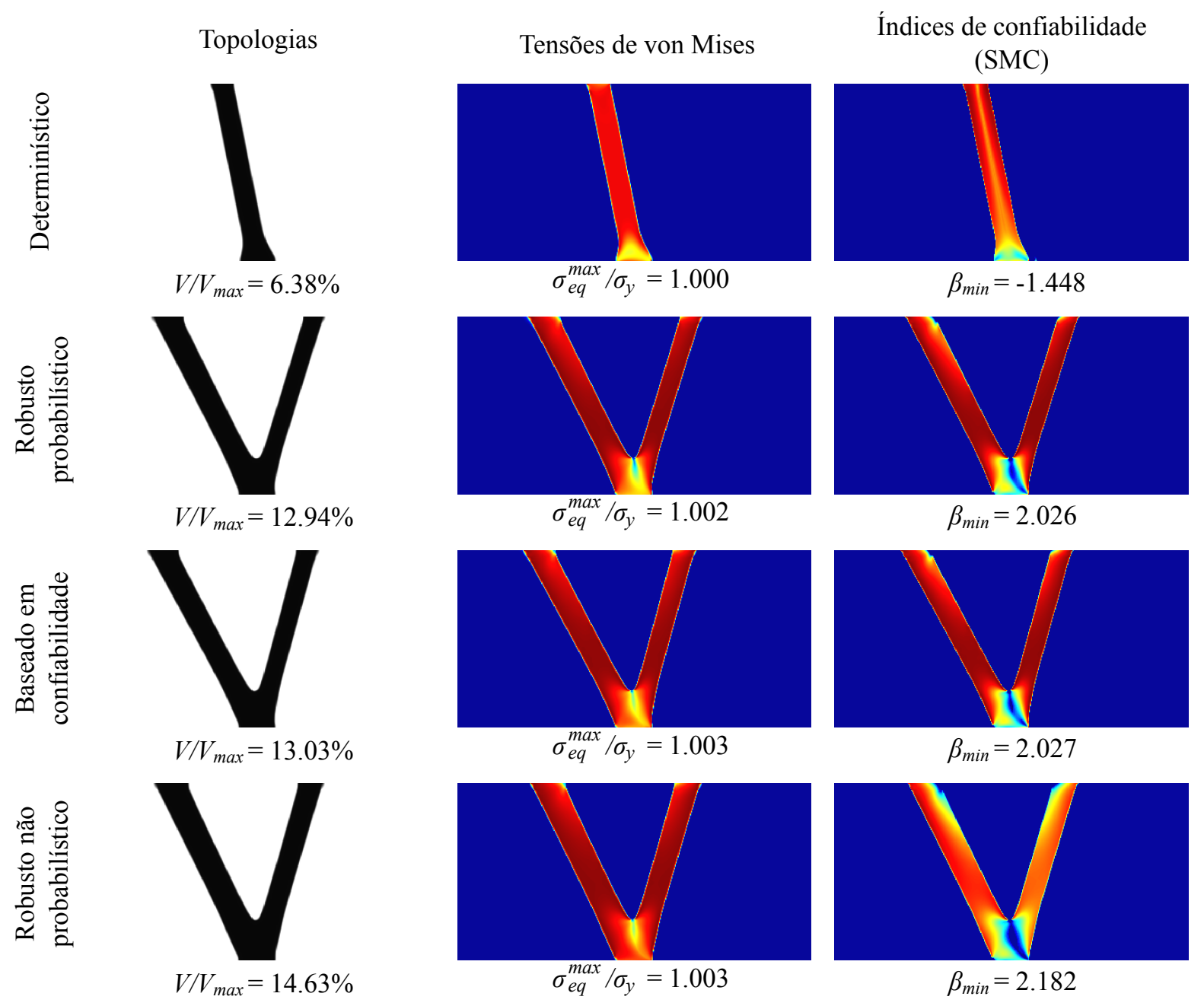

Fonte: produção do próprio autor.

Pode-se observar, na Figura 23, nos gráficos de tensão, que todas as estruturas estão altamente estressadas, dadas as medidas de tensão adotadas, e que as tensões máximas excedem a tensão limite em menos de 1\%, indicando que todas as estruturas estão verdadeiramente otimizadas dados os problemas de otimização considerados. Em outras palavras, não existe espaço para melhorias adicionais em relação ao volume estrutural, pois uma minimização de volume adicional implicaria em maiores tensões, e consequentemente, em resultados inviáveis.

Apesar das medidas de tensão das abordagens robusta probabilística e baseada em confiabilidade possuírem diferentes definições, as respectivas soluções são quase idênticas. Os índices de confiabilidade mínimos pós-processados são próximos de 2 em ambos os casos, indicando que a abordagem robusta probabilística, neste caso, pode ser empregada 
como uma alternativa a abordagem baseada em confiabilidade. Entretanto, embora muito similares neste caso, nada pode ser afirmado para outros problemas, especialmente quando variáveis não Gaussianas forem utilizadas na representação das variáveis incertas.

A abordagem robusta não probabilística, por outro lado, demonstra ser mais conservadora que as abordagens probabilísticas, uma vez que o seu índice de confiabilidade mínimo, $\beta_{\min }=2.182$, resultou ligeiramente maior que $\beta_{T}=2$. Isto é justificado, uma vez que a abordagem não probabilística garante viabilidade de tensão para todo o espaço de variáveis incertas dadas todas as combinações de valores extremos para as forças aplicadas. No caso baseado em confiabilidade, por outro lado, os pontos mais extremos não são considerados, pois as suas probabilidades de ocorrência são menores que a probabilidade de falha admissível. Neste caso, verifica-se que não existe relação direta entre abordagens probabilísticas e não probabilística, pois tratam-se de abordagens distintas.

O número de iterações é mostrado, para cada caso, na Tabela 2. Verifica-se que as abordagens não determinísticas requerem mais iterações até a convergência. Além disso, as abordagens não determinísticas exigem a solução de equações de equilíbrio adicionais, uma para cada força incerta, para a obtenção dos deslocamentos auxiliares e posterior cálculo das respectivas medidas de tensão. Adicionalmente, nas abordagens acopladas, problemas internos de otimização são solucionados para cada ponto de cálculo de tensão, no início de cada iteração. Entretanto, como o princípio de superposição de efeitos é empregado, o custo computacional para solucionar um dado problema interno (análise de confiabilidade inversa ou análise de pior cenário) é desprezível, uma vez que não é necessário solucionar as equações de equilíbrio para cada avaliação das tensões de von Mises, e suas derivadas em relação às variáveis incertas, a cada iteração dos problemas internos.

Apesar de não considerada em nossas implementações, o uso de computação paralela pode reduzir drasticamente o custo computacional associado a obtenção dos pontos de mínimo desempenho (abordagem baseada em confiabilidade) ou piores cenários das restrições de tensão (abordagem robusta não probabilística) no início de cada iteração. Uma vez que os problemas internos não dependem uns dos outros, estes podem ser solucionados de forma simultânea, de forma que o uso de computação paralela pode ser bastante efetivo na redução do custo computacional total, conforme demonstrado por Gurav, Goosen e vanKeulen (2005) no projeto de MEMS (MicroElectroMechanical Systems) sob variáveis incertas-mas-limitadas.

\subsubsection{Problema (L1) sob uma força incerta}

O problema (L1) abordado nesta subseção, Figura 22 (b), é discretizado com $N_{e}=57600$. Duas forças são aplicadas sobre o problema, uma horizontal e uma vertical. A força vertical é determinística, $f_{V}=0.3 \mathrm{~N}$, e a força horizontal possui direção determinística e intensidade incerta, $f_{H} \sim N(0,0.015) \mathrm{N}$. As forças aplicadas são distribuídas sobre um 
comprimento de $0.06 \mathrm{~m}$, para evitar concentrações de tensão.

De forma a comparar as abordagens determinística e não determinísticas, três problemas distintos são formulados, baseados em como a força horizontal é tratada:

- Problema determinístico, com força horizontal nula (valor médio);

- Problema probabilístico, considerando $f_{H} \sim N(0,0.015) N$. Dados adicionais: $\alpha=2$, abordagem robusta probabilística, Equação (5.2); $\beta_{T}=2$, abordagem baseada em confiabilidade, Equação (5.12);

- Problema não probabilístico, onde a intensidade incerta é limitada: $f_{H} \in[-0.03,0.03] \mathrm{N}$. Os limites são definidos considerando $\mathrm{E}[f] \pm 2 \times \operatorname{Std}[f]$ (uma vez que se considera $\alpha=\beta_{T}=2$ no problema probabilístico).

Os problemas de anti-otimização (problema robusto não probabilístico) são solucionados com a abordagem de duas etapas descrita na subseção 5.1.3. Para cada ponto de cálculo de tensão: 1) avalia-se a tensão equivalente de von Mises para 3 pontos $\left.\left(\mathbf{f}_{H}^{\text {grid }}=[-0.03,0,0.03]^{T} \mathrm{~N}\right) ; 2\right)$ partindo do ponto que fornece a tensão de von Mises máxima, emprega-se o minimizador local para a obtenção da solução do problema de anti-otimização.

A Figura 24 mostra os resultados obtidos, incluindo os índices de confiabilidade pós-processados. As topologias obtidas nos casos baseado em confiabilidade e robusto não probabilístico são iguais a topologia determinística. A topologia obtida no caso robusto probabilístico apresenta uma pequena diferença local, que consiste em um pequeno furo. Os índices de confiabilidade pós-processados mostram que a topologia determinística é extremamente sensível a força horizontal incerta considerada. Por outro lado, verifica-se que todas as abordagens não determinísticas fornecem resultados confiáveis, no sentido em que os índices de confiabilidade mínimos pós-processados resultam próximos do índice de confiabilidade alvo, ou seja, $\beta_{\text {min }} \cong \beta_{T}$.

Ao contrário do que acontece no problema sob duas forças incertas, subseção 7.1.2.1, o problema (L1) sob uma força incerta, quando abordado com a formulação robusta não probabilística, não fornece um resultado mais conservador que as demais abordagens não determinísticas. As três estruturas não determinísticas possuem volumes estruturais e índices de confiabilidade mínimos pós-processados similares. Isto é justificado, pelo fato de que agora uma única variável incerta está sendo considerada. Neste caso, os extremos do intervalo são também levados em conta na abordagem probabilística, uma vez que a probabilidade de ocorrência destes pontos coincide com a probabilidade associada ao índice de confiabilidade alvo, $\beta_{T}$.

É interessante observar, neste caso, que os índices de confiabilidade alvo pósprocessados são críticos (ou seja, próximos de $\beta_{\text {min }}$ ) em poucos pontos, diferente do que 
Figura 24: Resultados do problema (L1) sob uma força incerta: topologias (esquerda); tensões de von Mises (centro); índices de confiabilidade pós-processados com SMC (direita). As tensões ilustradas são as próprias medidas de tensão adotadas em cada problema.

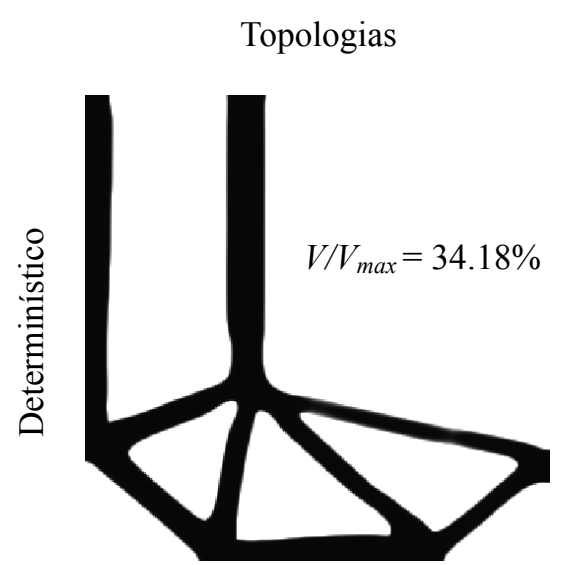

Tensões de von Mises

Índices de confiabilidade

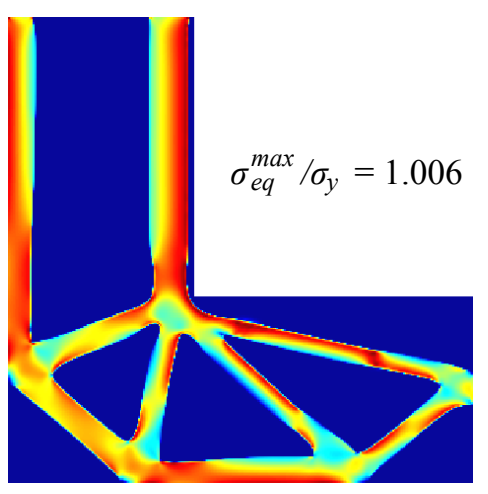
(SMC)
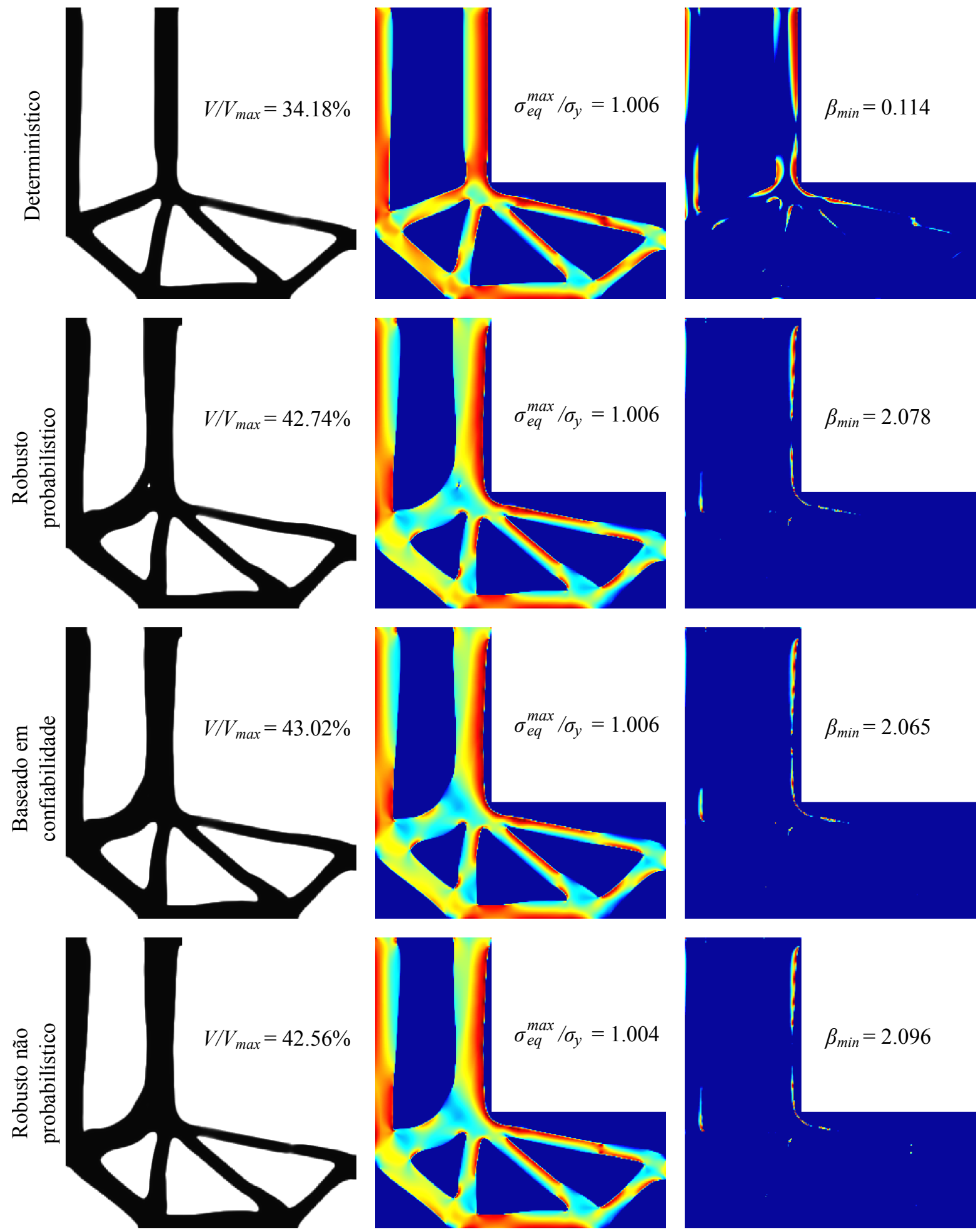

Fonte: produção do próprio autor.

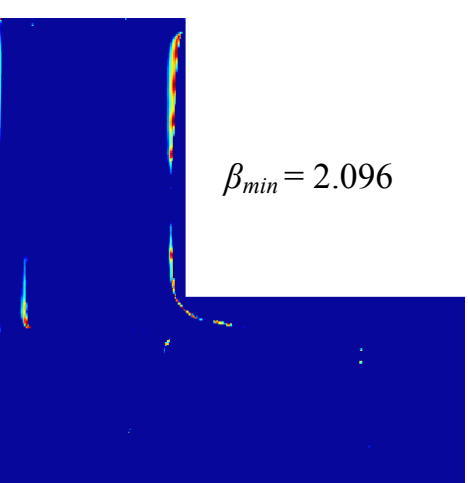

Fonte. produção do próprio autor. 
acontece no problema de domínio retangular, Figura 23, onde os índices pós-processados são críticos em quase toda a estrutura. Deve-se salientar que o problema de domínio retangular é bastante simples, de um ponto de vista de projeto, o que facilita a obtenção de estruturas quase totalmente estressadas. Problemas baseados em tensão com domínio em forma de L são mais desafiadores, de um ponto de vista de projeto, exigindo uma estrutura mais complexa, com menos pontos sob a tensão máxima, o que implica em gráficos de pós-processamento com menos pontos críticos.

A Tabela 3 mostra a probabilidade de falha máxima, associada ao índice de confiabilidade mínimo pós-processado por SMC, e o número de iterações até a convergência, para cada problema de otimização solucionado nesta subseção.

Tabela 3: Volumes estruturais, índices de confiabilidade mínimos, probabilidades de falha máximas e número de iterações até a convergência. Problema (L1) sob uma força incerta.

\begin{tabular}{lcccc}
\hline Problema & $V / V_{\max }$ & $\beta_{\min }$ & $P_{f}^{\max }$ & Iterações \\
\hline Determinístico & $34.18 \%$ & 0.114 & $45.47 \%$ & 1675 \\
Robusto prob. & $42.74 \%$ & 2.078 & $1.89 \%$ & 1848 \\
Baseado conf. & $43.02 \%$ & 2.065 & $1.94 \%$ & 1913 \\
Robusto não prob. & $42.56 \%$ & 2.096 & $1.80 \%$ & 2145 \\
\hline
\end{tabular}

Fonte: produção do próprio autor.

Comparando o número de iterações nas Tabelas 2 e 3, verifica-se que o problema (L1) exige um maior número de iterações até a convergência. Isto é justificável, uma vez que problemas baseados em tensão em forma de L são mais desafiadores, de um ponto de vista de projeto estrutural. A Tabela 3 mostra que as abordagens não determinísticas são mais custosas que a abordagem determinística, em concordância com os resultados da subseção anterior, mostrados na Tabela 2.

\subsubsection{Problema sob incerteza na direção das forças}

Esta subseção aborda o problema baseado em tensão sob incerteza na direção das forças aplicadas. As três formulações apresentadas na subseção 5.1 podem ser utilizadas neste caso; no entanto, como a direção é, por natureza, uma grandeza limitada, recomendase a solução destes problemas com a abordagem robusta não probabilística, Equação (5.18).

Nesta subseção, os problemas (L1) e (L2) são solucionados considerando uma força de intensidade determinística e direção incerta. Considera-se que a força pode ter qualquer inclinação, com $\theta \in[0,2 \pi]$ rad. O problema é solucionado com a abordagem robusta não probabilística e o resultado é comparado com a solução determinística para vários casos de carga. Os mesmos dados de entrada definidos na subseção 7.1.1 são aqui considerados. Uma malha com 57600 elementos é utilizada para discretizar o domínio de projeto. 
Ambos os problemas, (L1) e (L2), são solucionados considerando quatro cenários de carregamentos distintos, conforme ilustrado nas Figuras 25 e 26:

1. Uma força determinística vertical;

2. Quatro casos de carga determinísticos (não aplicados simultaneamente), defasados em $\pi / 2 \mathrm{rad}$;

3. Oito casos de carga determinísticos (não aplicados simultaneamente), defasados em $\pi / 4 \mathrm{rad}$

4. A direção da força é considerada desconhecida, e a anti-otimização é utilizada, considerando $\theta \in[0,2 \pi]$ rad.

As topologias otimizadas para quatro e oito casos de carga são obtidas considerando a formulação com restrição de tensão sob vários casos de carga (FANCELLO; PEREIRA, 2003), onde as restrições de tensão são satisfeitas para cada força separadamente. Consequentemente, estes problemas são solucionados para 230400 e 460800 restrições de tensão, respectivamente (57600 restrições de tensão para cada caso de carga).

Os problemas de anti-otimização são solucionados com a abordagem de duas etapas descrita na subseção 5.1.3. Para cada ponto de cálculo de tensão: 1) avalia-se a tensão equivalente de von Mises para 10 ângulos igualmente espaçados: $\boldsymbol{\theta}_{\text {grid }}=[\pi / 5,2 \pi / 5, \ldots, 9 \pi / 5,2 \pi]^{T}$ rad; 2) partindo da direção que fornece a tensão de von Mises máxima, emprega-se o minimizador local para a obtenção da solução do problema de anti-otimização.

Todas as soluções são pós-processadas considerando $\theta \in[0,2 \pi] \mathrm{rad}$, de forma a verificar as tensões de von Mises máximas para todas as direções possíveis que a força pode assumir. Os gráficos de pós-processamento também são ilustrados nas Figuras 25 e 26; estes são obtidos considerando $1 \times 10^{4}$ ângulos igualmente espaçados entre 0 e $2 \pi$ rad.

Analisando as topologias e as tensões de von Mises máximas pós-processadas, nas Figuras 25 e 26, verifica-se que a tensão de von Mises máxima normalizada de cada gráfico, $\max \left(\sigma_{e q}^{\max } / \sigma_{y}\right)$, diminui com o aumento de casos de carga, até o caso limite de otimização com anti-otimização. Por outro lado, verifica-se que o volume estrutural também aumenta com o aumento de casos de carga. Isto faz sentido, de um ponto de vista de engenharia estrutural, uma vez que mais material é necessário para garantir a viabilidade em tensão para um maior número de casos de carga.

As topologias obtidas para um único caso de carga, em ambos os problemas (L1) e (L2), não possuem material entre os membros verticais, ao contrário das outras topologias. As topologias para quatro e oito casos de carga, em ambos os problemas (L1) e (L2), são as mesmas, apresentando um único membro entre os membros verticais, tendo diferenças somente em suas formas. A solução robusta, no caso (L1), apresenta dois membros cruzados 
Figura 25: Topologias otimizadas obtidas como solução do problema (L1) considerando, da direita para a esquerda, otimização com: 1 força determinística; 4 casos de força determinísticos; 8 casos de força determinísticos; anti-otimização com $\theta \in[0,2 \pi] \operatorname{rad}$.
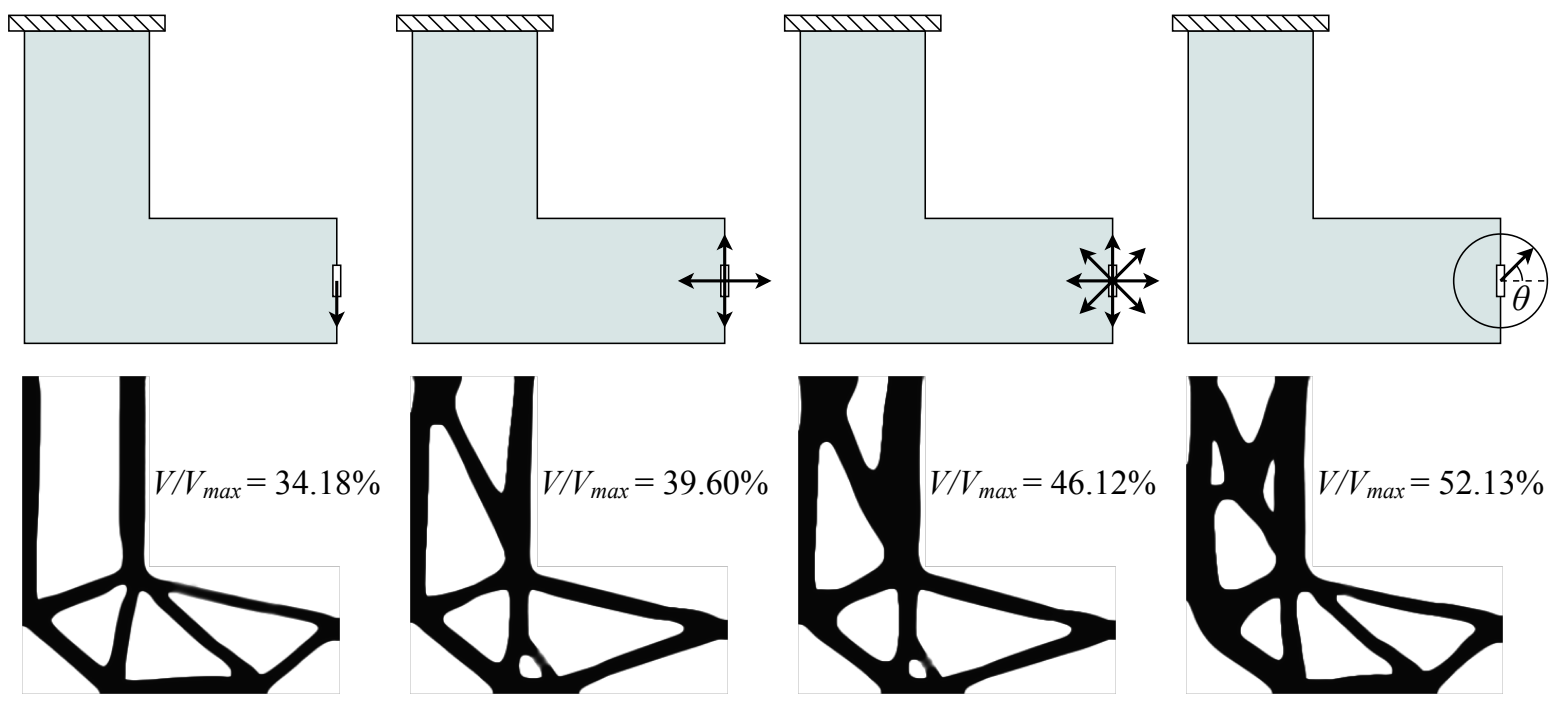

$\max \left(\sigma_{e q}^{\max } / \sigma_{y}\right)=5.841$

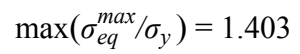

$$
\max \left(\sigma_{e q}^{\max } / \sigma_{y}\right)=1.074
$$

$\max \left(\sigma_{e q}^{\max } / \sigma_{y}\right)=1.004$
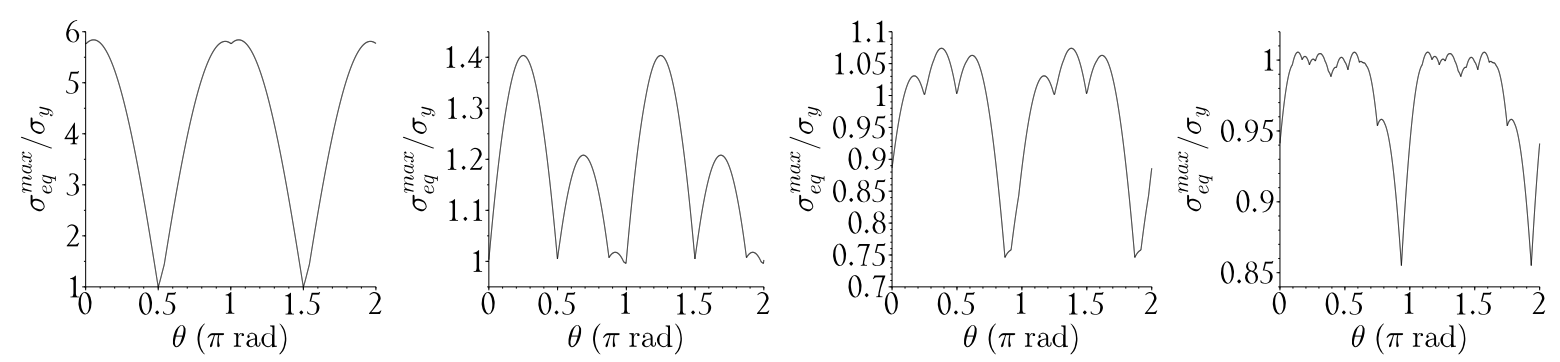

Fonte: Silva, Cardoso e Beck (2018).

entre os verticais, enquanto que a solução robusta no caso (L2) apresenta um único membro, como os resultados para quatro e oito casos de carga.

Estes resultados demonstram como as soluções determinísticas são instáveis e dependentes dos parâmetros utilizados na otimização. Mesmo quando vários casos de carga são considerados (até oito casos de carga, nestes exemplos), a viabilidade em tensão só é garantida para as direções das forças consideradas durante a otimização, como pode ser claramente verificado, nas Figuras 25 e 26, para quatro casos de carga, onde as restrições de tensão são satisfeitas para $\theta=0.0 \pi, 0.5 \pi, 1.0 \pi$ e $1.5 \pi$ rad. Qualquer perturbação na direção dos casos de carga poderia causar a falha da estrutura, conforme demonstrado no pós-processamento. As únicas topologias que se mostram verdadeiramente robustas são as soluções dos problemas de otimização com anti-otimização, onde as tensões máximas pós-processadas resultaram em 1.004, problema (L1), e 1.009, problema (L2), excedendo a tensão limite em $0.4 \%$ e $0.9 \%$, respectivamente, ou seja, respeitando a tolerância de $t_{\sigma} l_{\sigma}=1 \%$ considerada na solução dos problemas de otimização.

Além disso, a solução dos problemas determinísticos considerando vários casos de 
Figura 26: Topologias otimizadas obtidas como solução do problema (L2) considerando, da direita para a esquerda, otimização com: 1 força determinística; 4 casos de força determinísticos; 8 casos de força determinísticos; anti-otimização com $\theta \in[0,2 \pi] \mathrm{rad}$.
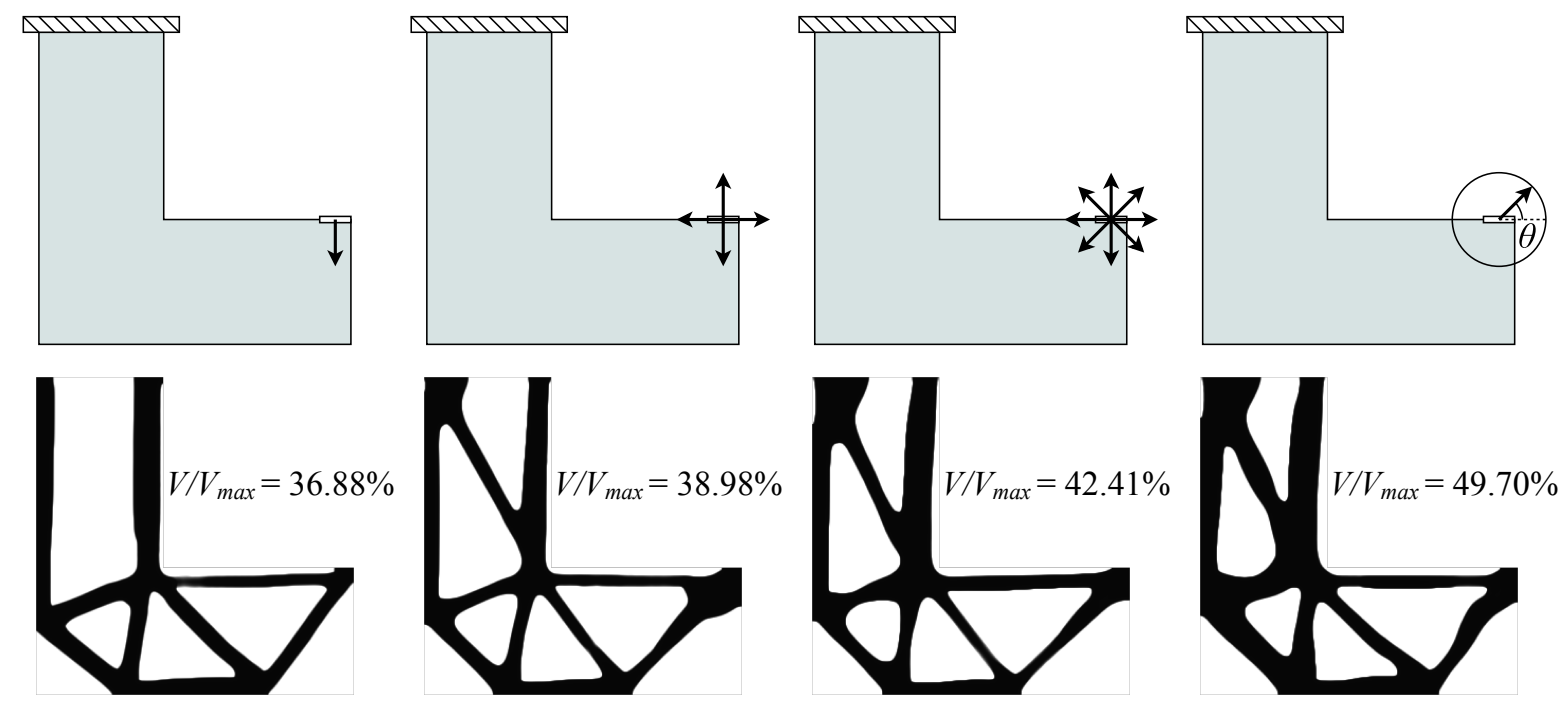

$\max \left(\sigma_{e q}^{\max } / \sigma_{y}\right)=5.448$

$$
\max \left(\sigma_{e q}^{\max } / \sigma_{y}\right)=1.387
$$

$$
\max \left(\sigma_{e q}^{\max } / \sigma_{y}\right)=1.081
$$
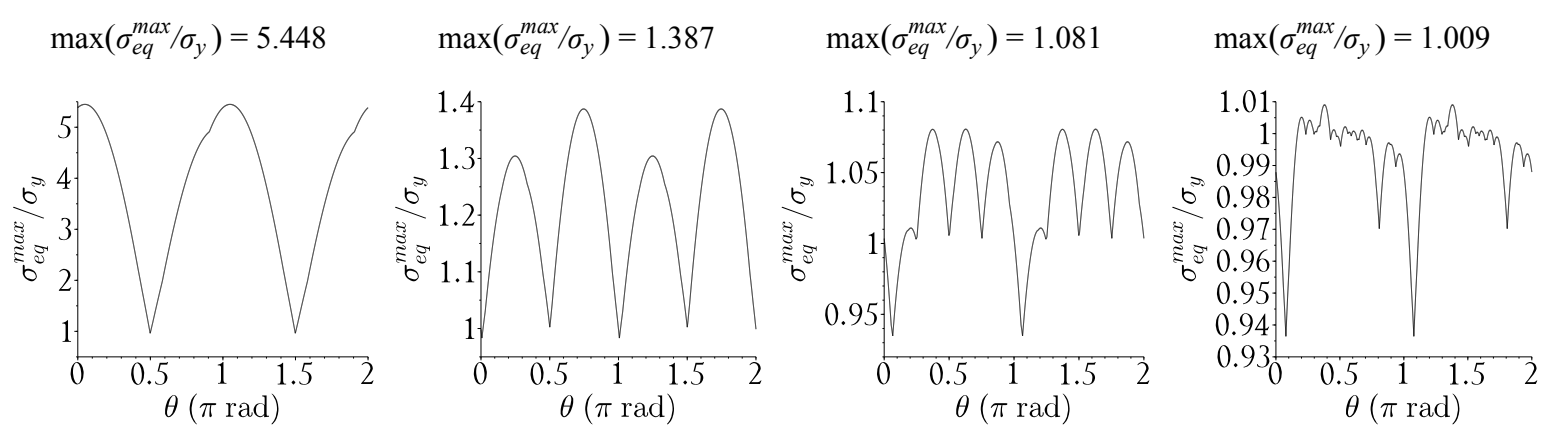

Fonte: Silva, Cardoso e Beck (2018).

carga é bastante difícil, devido a necessidade de ajustar o parâmetro de penalização associado ao método do Lagrangiano aumentado. Neste trabalho, o parâmetro de penalização $r$ e o seu valor máximo $r_{\max }$ são divididos pelo número de casos de carga, de forma a garantir subproblemas de otimização bem condicionados.

A Tabela 4 mostra o número total de iterações necessárias para convergência, para os problemas (L1) e (L2). Verifica-se que o número total de iterações para os problemas robustos é quase duas vezes o número de iterações para os problemas determinísticos, considerando um caso de carga. Este número de iterações adicionais é justificado, pois um problema muito mais desafiador é abordado neste caso, onde cada restrição de tensão deve ser satisfeita para a pior direção, dado um intervalo contínuo de direções.

Em relação ao custo computacional total, os problemas robustos são certamente mais custosos que o problema determinístico tradicional, devido a necessidade de solucionar equações de equilíbrio adicionais e problemas de anti-otimização a cada iteração do problema de otimização. Entretanto, conforme discutido previamente na subseção 7.1.2.1, o uso da superposição de efeitos e computação paralela pode ser feito de forma reduzir o 
Tabela 4: Número de iterações até a convergência, problemas determinísticos e robustos (L1) e (L2).

\begin{tabular}{lcc}
\hline Casos de carga & Problema (L1) & Problema (L2) \\
\hline 1 & 1675 & 1308 \\
4 & 2479 & 2832 \\
8 & 3153 & 3731 \\
Robusto & 2583 & 2595 \\
\hline
\end{tabular}

Fonte: adaptado de Silva, Cardoso e Beck (2018).

custo computacional total.

\subsection{Problema com restrição de tensão sob incerteza de manufatura}

De forma a demonstrar a aplicabilidade da formulação proposta, Equação (5.20), o problema (L2) é solucionado, nesta subseção, conforme Figura 27. A diferença entre o problema ilustrado na Figura 27 e aquele ilustrado na Figura 16 consiste em uma extensão do domínio de filtragem, de largura igual ao raio do filtro, preenchida com elementos vazios. A extensão do domínio de filtragem é apresentada por Clausen e Andreassen (2017) como uma técnica para reduzir possíveis efeitos de contorno do domínio de projeto sobre a topologia otimizada, que podem ocorrer devido a aplicação do filtro de densidades.

Figura 27: Problema (L2) considerado nesta subseção. Dimensões em metro e força em Newton.

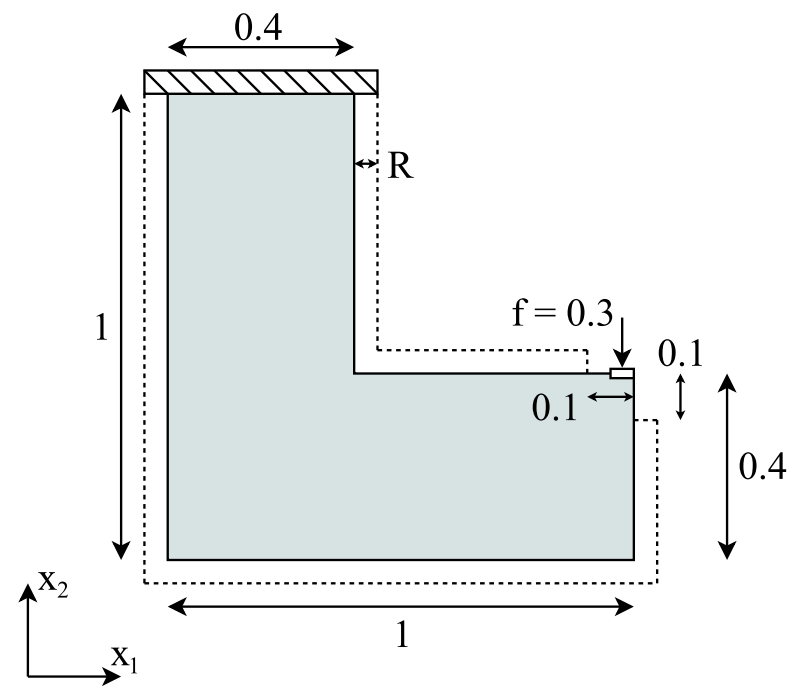

Fonte: Silva, Beck e Sigmund (2019).

Utilizam-se os mesmos dados de entrada definidos na subseção 7.1.1, com exceção dos parâmetros definidos neste parágrafo: $r^{(1)}=0.003$ e $r_{\max }=3000$, associados ao método do Lagrangiano aumentado; $n i t_{\max }=20$ como número máximo de iterações de cada subproblema de otimização; utilizam-se a função de interpolação de tensão $f_{\sigma}^{\varepsilon}$, com $\varepsilon=0.2$, e a função de projeção parametrizada, Equação $(2.11)$, com $\delta_{\text {max }}=\delta_{\text {lim }} / 2$, de 
forma a permitir uma fina camada de material intermediário entre as fases sólida e vazia da topologia, com o intuito de garantir acurácia no cálculo das tensões após variação uniforme de contorno.

Discretiza-se o domínio de projeto com uma malha de $400 \times 400$ elementos $\left(N_{e}=\right.$ 102400). Com a Equação (5.27), calcula-se $\delta_{\text {lim }}=2 \times R / l_{e}=2 \times 0.02 /(1 / 400)=16$. Utiliza-se uma abordagem de continuação de três passos para a atualização do parâmetro de projeção $\delta$, até o seu valor máximo de $\delta_{\max }=\delta_{\text {lim }} / 2=8$, como: $\delta^{(1)}=1, \delta^{(2)}=4 \mathrm{e}$ $\delta^{(3)}=8$.

Neste exemplo, as topologias dilatada, intermediária e erodida são avaliadas para $\eta_{d}=0.25, \eta_{i}=0.5$ e $\eta_{e}=0.75$, respectivamente. A solução otimizada é obtida em 1222 iterações. A Figura 28 mostra os três campos otimizados de densidades relativas e as respectivas tensões equivalentes de von Mises.

Figura 28: Topologias e respectivas tensões equivalentes de von Mises para estruturas otimizadas erodida (esquerda), intermediária (centro) e dilatada (direita).

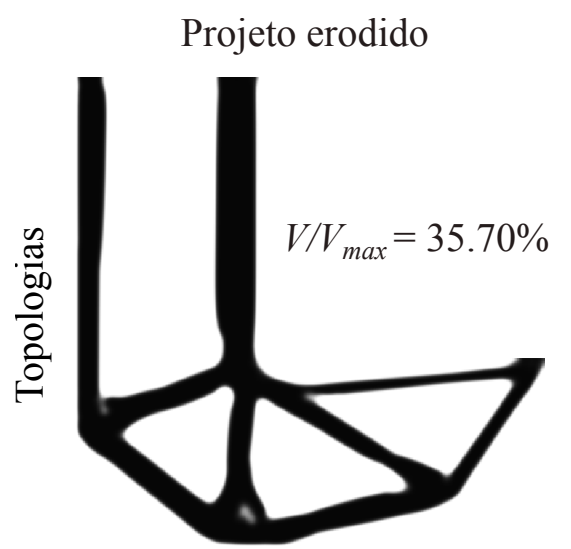

$$
\text { Projeto intermediário }
$$

Projeto dilatado
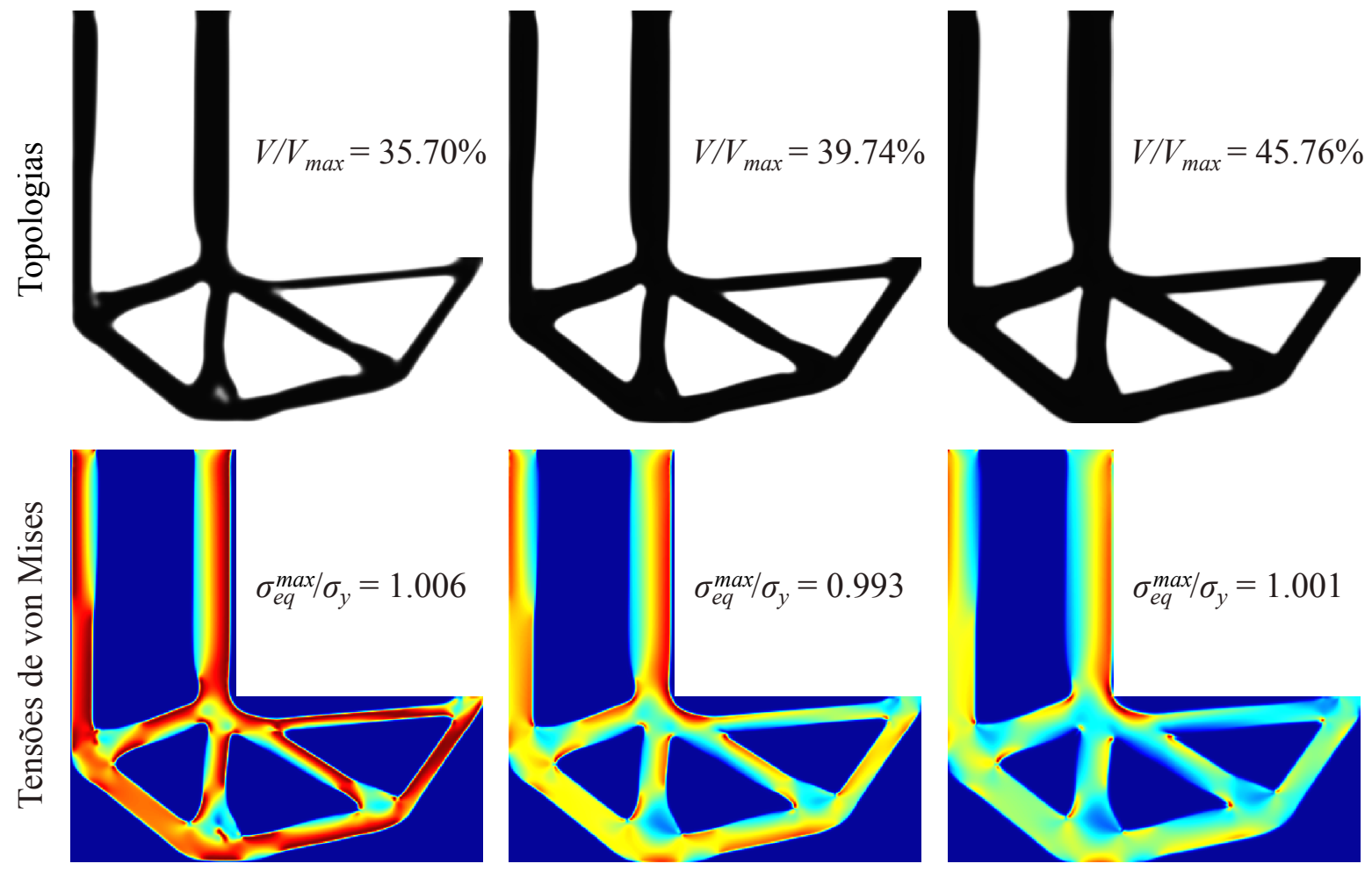

Fonte: adaptado de Silva, Beck e Sigmund (2019).

Analisando a Figura 28, verifica-se que as topologias erodida, intermediária e dilatada são quase as mesmas. A única diferença entre elas está na topologia erodida, que apresenta um pequeno furo. A Figura 28 também mostra que as três estruturas satisfazem o critério de falha em tensão em todos os pontos onde a tensão de von Mises é calculada, considerando a tolerância estabelecida de $t o l_{\sigma}=1 \%$ em relação a tensão limite. Conforme 
esperado, a estrutura erodida é um sistema muito estressado, enquanto a estrutura dilatada apresenta menos pontos sob a tensão máxima. É interessante observar que existe uma tendência para evitar o canto vivo do domínio de projeto, em cada um dos três campos de densidades relativas. A influência do pequeno furo sobre a distribuição de tensão da topologia erodida é verificada posteriormente, durante o pós-processamento com malhas alinhadas ao corpo.

Apesar das estruturas erodida, intermediária e dilatada respeitarem as restrições de tensão, a avaliação das tensões de von Mises para valores de $\eta$ entre $\eta_{d}$ e $\eta_{e}$ é necessária, para verificar se a estrutura intermediária é verdadeiramente robusta em relação a variações uniformes de contorno. Além disso, deve-se efetuar um pós-processamento com malhas alinhadas ao corpo, para verificar se as tensões obtidas com o modelo baseado em pixel (aqui baseado em pixel, por ser um estudo bidimensional) coincidem com as tensões obtidas através do modelo ajustado ao corpo (topologia suave subjacente).

Consequentemente, dois esquemas de pós-processamento são empregados e posteriormente comparados: 1) esquema de pós-processamento baseado em pixel; 2) esquema de pós-processamento ajustado ao corpo.

O primeiro e mais simples procedimento de pós-processamento aqui empregado é o esquema baseado em pixel. Para $\eta \in[0.0,0.8]$, considerando passos incrementais de 0.001, aplica-se a função de projeção parametrizada, para $\delta=8$ (valor máximo considerado durante a otimização), sobre as variáveis filtradas otimizadas, $\tilde{\boldsymbol{\rho}}^{*}$, e calcula-se a máxima tensão equivalente de von Mises. Este procedimento fornece um gráfico $\eta \times \sigma_{e q}^{\max } / \sigma_{y}$, que é posteriormente analisado junto aos resultados do esquema de pós-processamento ajustado ao corpo. O intervalo $\eta \in[0.0,0.8]$ é intencionalmente investigado, pois ele contém os valores limites de $\eta_{d}=0.25$ e $\eta_{e}=0.75$, empregados durante a otimização. Valores de $\eta$ maiores que 0.8 não são utilizados no pós-processamento, pois estruturas cada vez mais finas são obtidas com o aumento do valor de $\eta$, levando a estruturas desconectadas e valores extremamente altos de tensão, escondendo completamente as menores contribuições de tensão no gráfico.

O segundo procedimento de pós-processamento, o esquema ajustado ao corpo, também é realizado para $\eta \in[0.0,0.8]$. Entretanto, como uma malha não estruturada específica é necessária para cada pequena variação de forma/topologia, um menor número de valores de $\eta$ é considerado. Este estudo é então realizado para dezesseis valores de $\eta$, de $\eta=0.05$ até $\eta=0.8$, considerando passos incrementais de 0.05 .

O seguinte procedimento é adotado, de forma a obter estruturas suaves das topologias baseadas em pixel, e posteriormente obter malhas não estruturadas ajustadas aos corpos destas estruturas:

1. Uma simples média nodal é efetuada sobre o campo otimizado de densidades filtradas, 
incluindo os elementos vazios presentes nas áreas de extensão do filtro de densidades;

2. Para um dado valor de $\eta$, a topologia suave subjacente é diretamente obtida das suas curvas de nível, extraídas diretamente da média nodal do campo de densidades filtradas;

3. Uma malha refinada não estruturada, com elementos de segunda ordem triangulares de seis nós, é gerada sobre as curvas de nível previamente obtidas, utilizando-se o software Gmsh (GEUZAINE; REMACLE, 2009).

O procedimento é ilustrado na Figura 29.

Figura 29: Procedimento empregado para extração de contorno suave das topologias otimizadas.

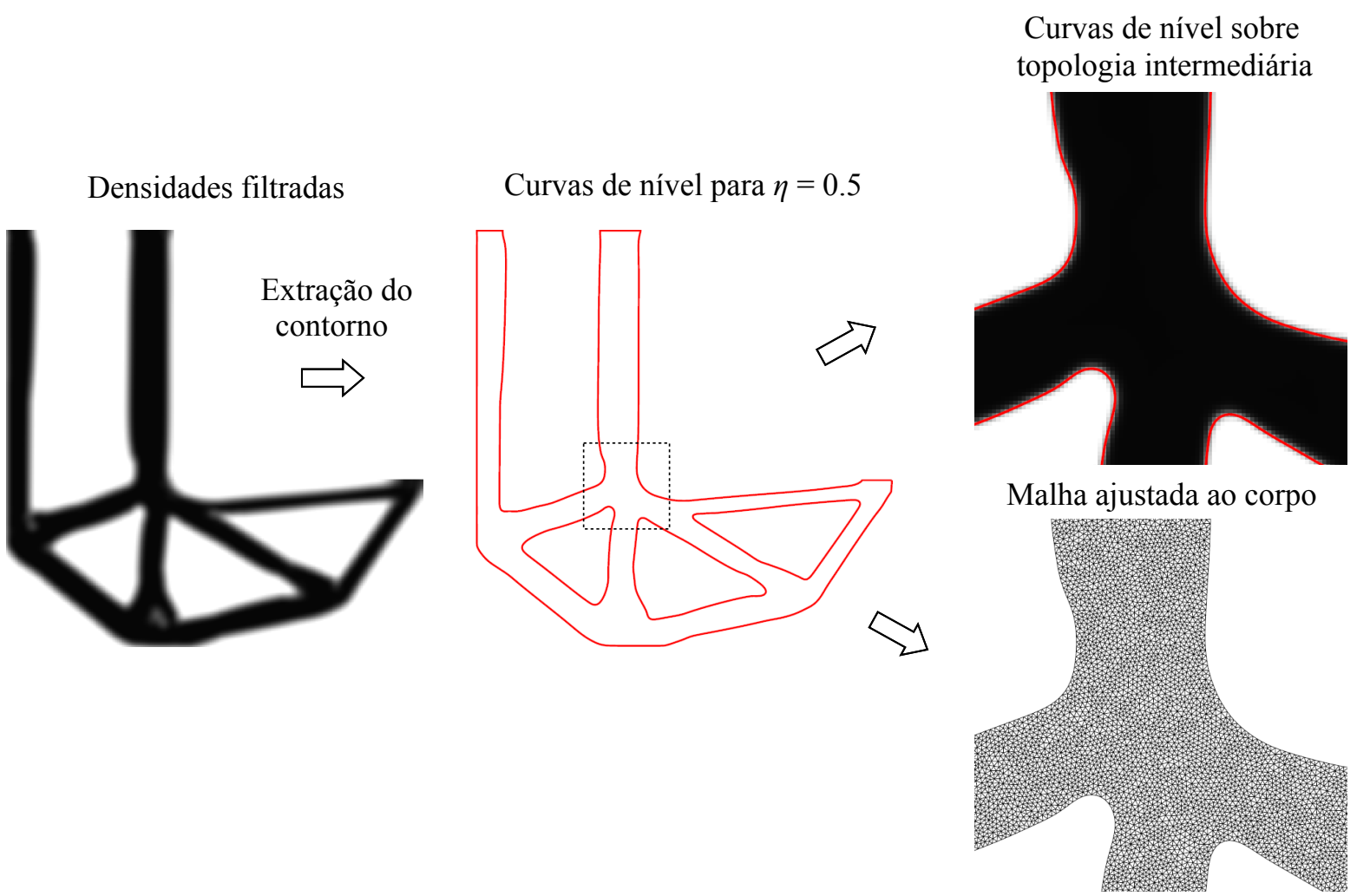

Fonte: adaptado de Silva, Beck e Sigmund (2019).

Após a etapa de geração de malha, as tensões equivalentes de von Mises são avaliadas no centroide de cada elemento triangular. O número de elementos em cada malha varia, sendo em média 78000.

A Figura 30 mostra os resultados obtidos para os dois procedimentos de pósprocessamento empregados.

Analisando a Figura 30, pode-se verificar variação de tensão suave entre $\eta_{d}=0.25$ e $\eta_{e}=0.75$, considerando ambos os modelos baseado em pixel e ajustado ao corpo. Considerando o modelo baseado em pixel (linha sólida), observa-se que o valor da tensão 
Figura 30: Tensões de von Mises máximas pós-processadas. Resultados referentes às estruturas erodida, intermediária e dilatada, considerando o modelo ajustado ao corpo, são indicados por círculos.

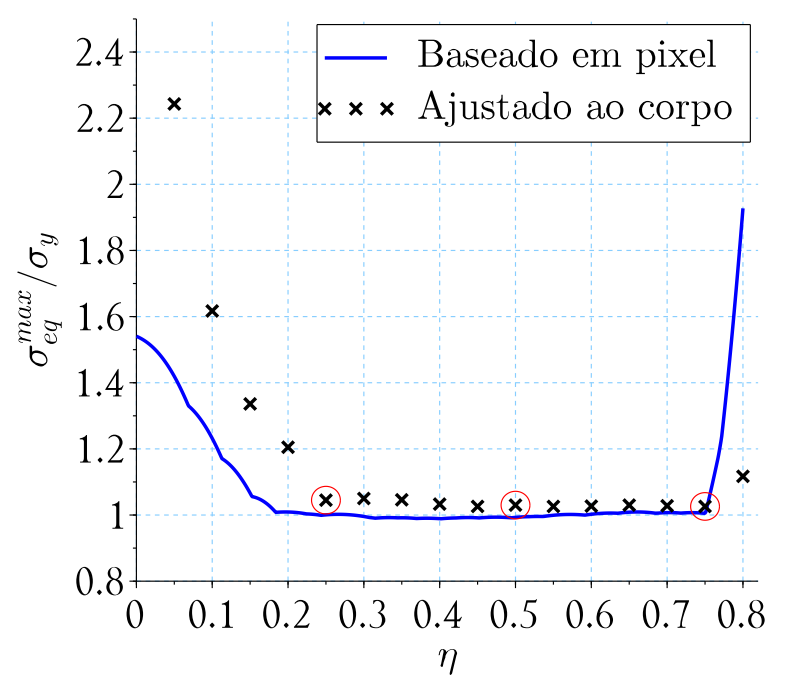

Fonte: adaptado de Silva, Beck e Sigmund (2019).

máxima normalizada é próximo de 1 no intervalo entre $\eta_{d}=0.25$ e $\eta_{e}=0.75$. É também importante observar que, apesar de diferentes, as respostas obtidas com a abordagem ajustada ao corpo, entre $\eta_{d}=0.25$ e $\eta_{e}=0.75$, são próximas das respostas obtidas com a abordagem baseada em pixel, excedendo a restrição de tensão em apenas 5\%. Consequentemente, verifica-se que a abordagem proposta, Equação (5.20), é adequada para obtenção de estruturas realmente robustas a variações uniformes de contorno, quando valores apropriados de $\delta_{\max }$ (projeção parametrizada) e $\varepsilon$ (interpolação de tensão) são escolhidos.

Na estrutura erodida, Figura 28 (esquerda), existe um pequeno furo, indicando uma diferença local entre as topologias erodida e intermediária. Como esperado, este pequeno furo também está presente nas curvas de nível associadas a topologia erodida $(\eta=0.75)$, Figura 31 (a) e consequentemente, no modelo de elementos finitos ajustado ao corpo, Figura 31 (c). De forma a verificar a influência desta diferença sobre a tensão de von Mises máxima, o mesmo procedimento de pós-processamento com malha ajustada ao corpo é realizado desconsiderando o pequeno furo, Figura 31 (b), onde uma tensão máxima de 1.026 é obtida, Figura 31 (d), ou seja, não existe diferença entre as tensões máximas no projeto erodido com ou sem o pequeno furo. Consequentemente, neste exemplo, esta diferença entre as topologias erodida e intermediária não influencia na robustez do projeto intermediário.

Fora do limite de $\eta$ considerado na otimização, ou seja, para $\eta<0.25$ e $\eta>0.75$, diferentes comportamentos são observados. Nestes casos, o modelo é muito grosso (levando a estruturas com um canto vivo de singularidade de tensão) ou muito fino. Entretanto, 
Figura 31: Curvas de nível, estrutura erodida: (a) considerando o furo; (b) desconsiderando o furo; e respectivas tensões equivalentes de von Mises resultantes de modelos ajustados ao corpo: (c) e (d).

Curvas de nível para $\eta=0.75$

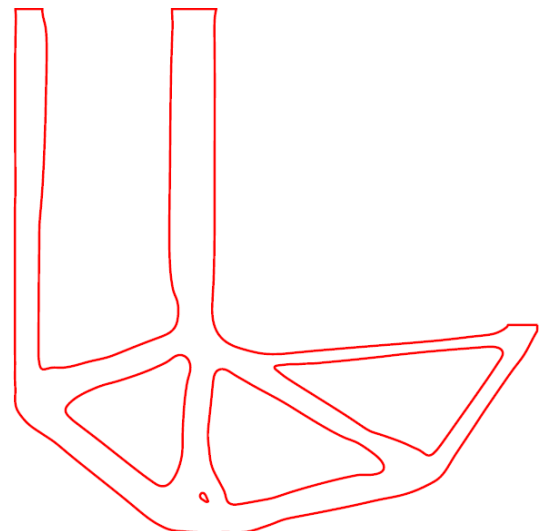

(a)

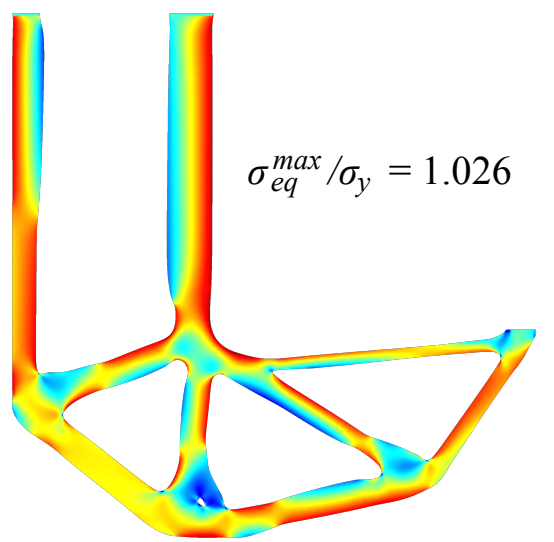

(c)
Curvas de nível para $\eta=0.75$ desconsiderando o furo

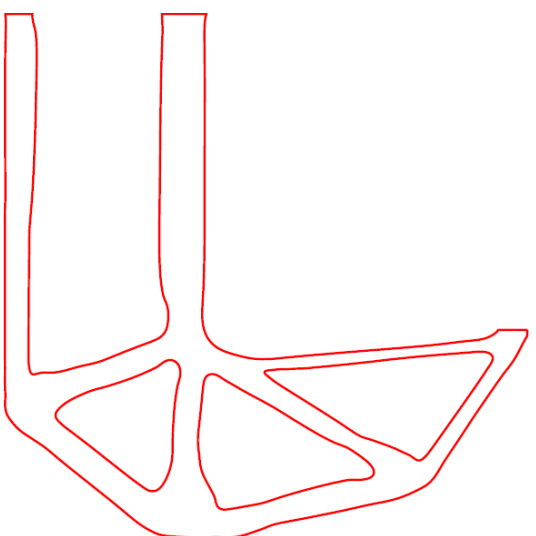

(b)

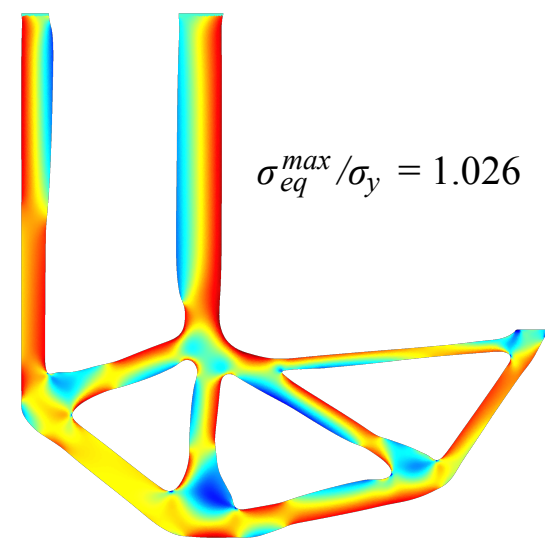

(d)

Fonte: adaptado de Silva, Beck e Sigmund (2019).

ambos os casos estão fora do escopo da otimização e não alteram a acurácia dos resultados obtidos.

Apesar de boa concordância ser observada entre as tensões de von Mises máximas, para $\eta \in[0.25,0.75]$, é também interessante observar a similaridade das distribuições de tensão resultantes das análises baseadas em pixel e ajustadas ao corpo. A Figura 32 mostra as distribuições de tensão de von Mises obtidas com os modelos baseado em pixel e ajustado ao corpo, para $\eta=0.5$. Apesar das tensões máximas serem ligeiramente diferentes, as distribuições de tensão são bastante similares, devido a utilização bem sucedida do parâmetro limite de projeção, $\delta_{l i m}$, associada a escolha razoável do parâmetro de interpolação de tensão.

Note que a distribuição de tensão baseada em pixel tem um contorno de transição entre as fases sólida e vazia, ou seja, existe uma transição de tensão suave das tensões 
Figura 32: Tensões equivalentes de von Mises obtidas para os modelos baseado em pixel (esquerda) e ajustado ao corpo (direita), para $\eta=0.5$.
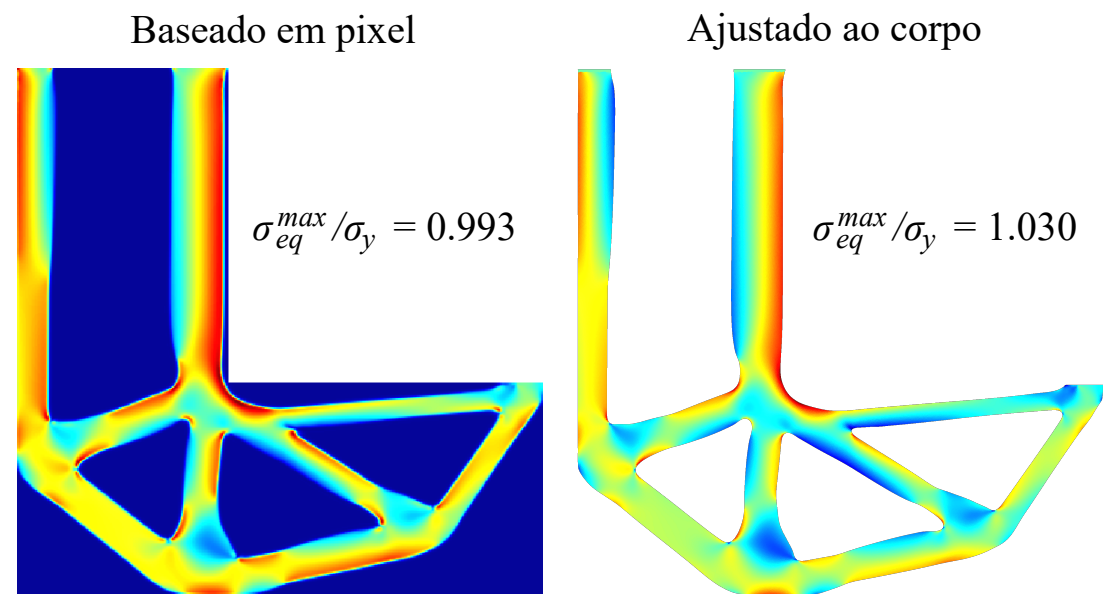

Fonte: adaptado de Silva, Beck e Sigmund (2019).

finitas, presentes na fase sólida, para as tensões nulas, presentes na fase vazia. Este fenômeno, que também é observado nas estruturas erodida e dilatada, Figura 28, é uma consequência direta da utilização de uma fina camada de material de transição entre as fases sólida e vazia da topologia. Apesar destas tensões de transição não possuírem significado físico, pode-se verificar que elas não afetam a qualidade da distribuição de tensão, uma vez que as tensões dos modelos baseado em pixel e ajustado ao corpo são bastante similares, com exceção do fato do modelo ajustado ao corpo não possuir tais efeitos de transição de contorno, Figura 32. Além disso, as camadas de transição entre as fases sólida e vazia, no modelo baseado em pixel, podem ser propriamente reduzidas com refino da malha, o que reduziria o tamanho dos elementos e, consequentemente, aumentaria o valor de $\delta_{l i m}$. Maiores valores de $\delta_{\text {lim }}$ permitiriam a utilização de maiores valores de $\delta_{\max }$ na projeção parametrizada, sem os efeitos indesejados de oscilação de tensão devido a variações uniformes de contorno; assim mantendo uma resposta de tensão suave para menores larguras de transição de material intermediário, considerando as distribuições de tensão dos modelos baseados em pixel.

Também é notável, embora não seja inesperado neste exemplo, que a otimização resulte em uma estrutura que mantém tensão de von Mises máxima constante após variações uniformes de contorno; consequentemente fornecendo um projeto verdadeiramente robusto em relação à tensão máxima. Neste aspecto, o projeto baseado em tensão é bastante diferente do projeto baseado em flexibilidade. Conforme demonstrado em Sigmund (2009), a estrutura erodida é sempre a mais crítica no projeto baseado em flexibilidade. O projeto baseado em tensão, por outro lado, apresenta um comportamento bastante diferente, onde as restrições de tensão estão ativas em todo o intervalo de $\eta$ considerado na otimização, indicando que todas as estruturas para $\eta$ entre $\eta_{d}$ e $\eta_{e}$ são críticas.

É importante enfatizar que o resultado obtido com esta formulação, Equação 
(5.20), se mostrou robusto em relação a incerteza uniforme de manufatura somente. Caso variações não uniformes de contorno ocorram, nada pode ser afirmado sobre o desempenho da estrutura obtida.

\subsubsection{Influência do tamanho da malha}

De forma a verificar a influência do tamanho da malha sobre a qualidade da solução robusta, o problema (L2), Figura 27, é solucionado com uma malha mais grosseira, com 25600 elementos (grade de $200 \times 200$ elementos). O problema é solucionado para $\delta_{\max }=8$, que coincide com o valor de $\delta_{l i m}$ neste caso. A solução otimizada é obtida em 911 iterações.

A Figura 33 mostra as topologias intermediárias, para malhas grosseira $\left(N_{e}=\right.$ $25600)$ e refinada $\left(N_{e}=102400\right)$. Detalhes dos cantos reentrantes são mostrados, onde as densidades filtradas são ilustradas junto às curvas de nível suaves, utilizadas no pósprocessamento com malhas ajustadas ao corpo.

Figura 33: Topologias intermediárias (robustas) e detalhes dos cantos reentrantes com densidades filtradas e curvas de nível consideradas no pós-processamento, para $N_{e}=25600$ (esquerda) e $N_{e}=102400$ (direita).
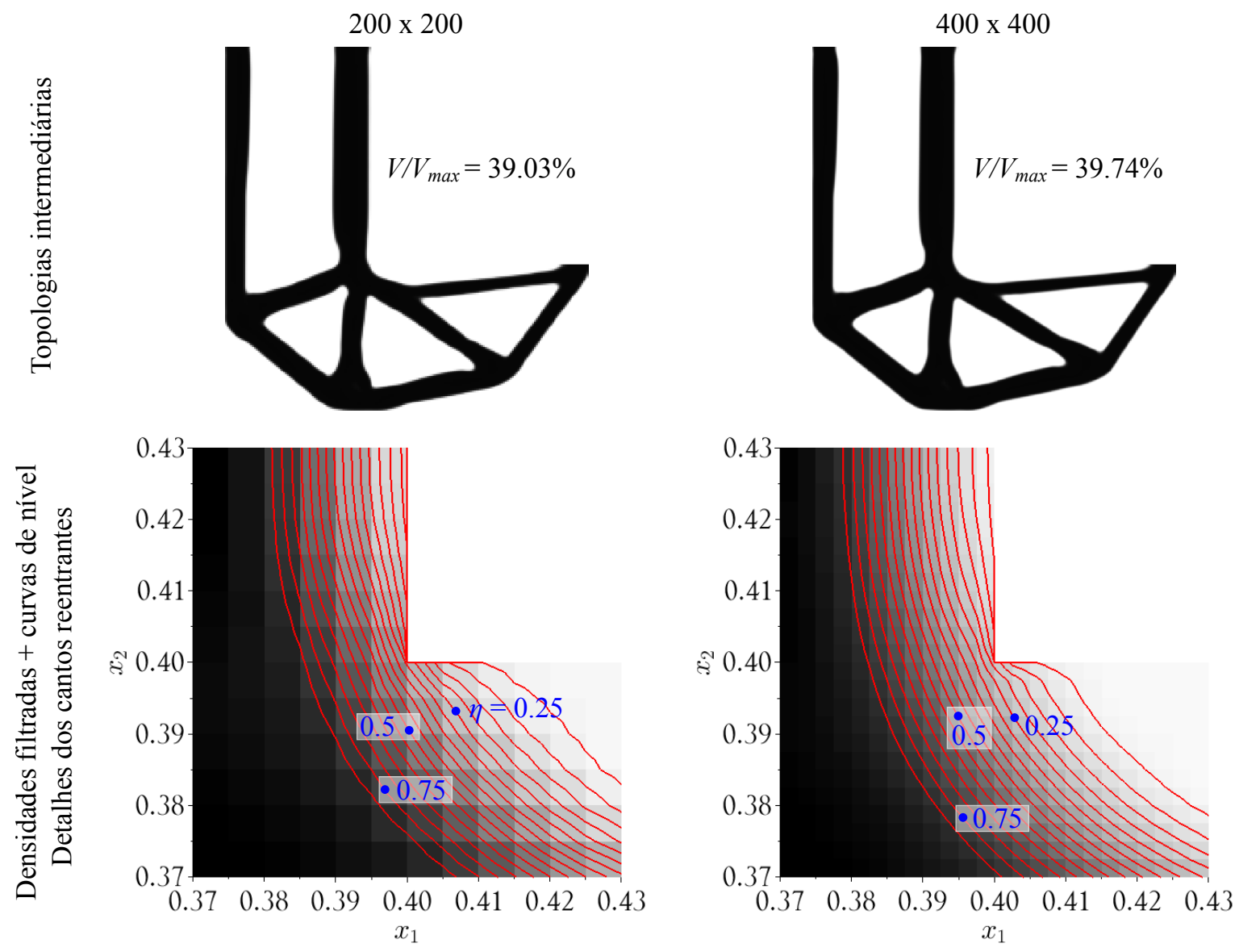

Fonte: adaptado de Silva, Beck e Sigmund (2019).

Apesar de topologias idênticas serem obtidas após a otimização, pequenas diferenças em suas formas são observadas. Analisando as curvas de nível sobre as densidades filtradas, 
verificam-se duas diferenças entre as soluções para malhas grosseira e refinada: 1) as curvas de nível referentes à solução com malha refinada são mais suaves; e 2) a curva de nível para $\eta=0.25$ (projeto dilatado), considerando a malha grosseira, possui um pequeno canto reentrante.

Apesar destas diferenças, a solução otimizada com malha grosseira também apresenta bons resultados de pós-processamento, dentro do intervalo $\eta \in[0.25,0.75]$, conforme ilustrado na Figura 34, com exceção do resultado para $\eta=0.25$ obtido com o modelo ajustado ao corpo, que apresenta um canto reentrante.

Figura 34: Tensões de von Mises máximas pós-processadas, para $N_{e}=25600$. Resultados referentes às estruturas erodida, intermediária e dilatada, considerando o modelo ajustado ao corpo, são indicados por círculos.

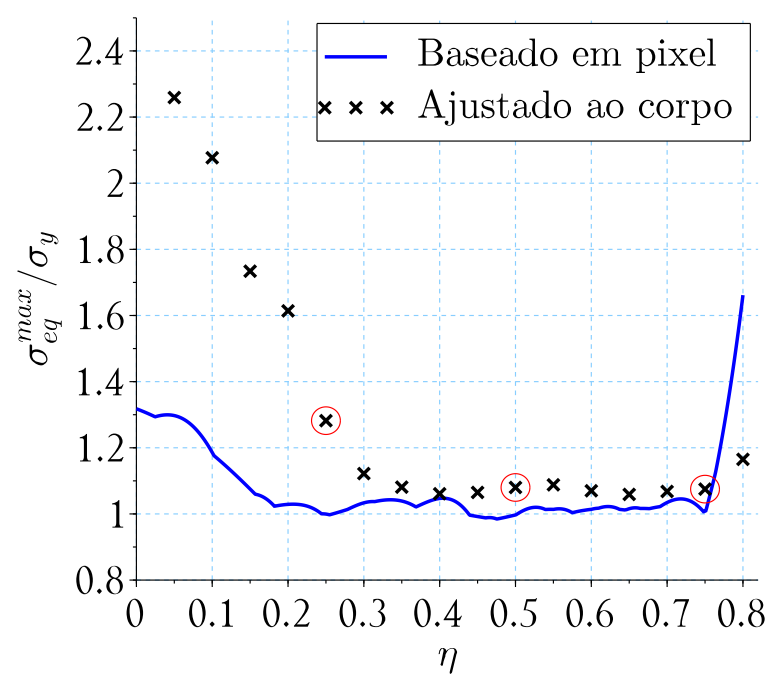

Fonte: adaptado de Silva, Beck e Sigmund (2019).

As tensões de von Mises máximas, do modelo baseado em pixel, oscilam com a variação do valor de $\eta$, de 0.25 até 0.75 . Este comportamento oscilatório é justificado, pois para a malha mais grosseira, o valor de $\delta_{\max }$, utilizado na projeção parametrizada, coincide com o valor de $\delta_{\text {lim }}$. Conforme mostrado na Figura 14, para $\varepsilon=0.2$ e $\delta=\delta_{\text {lim }}$, pequenas oscilações de tensão podem ocorrer, independente da discretização. Apesar destas oscilações, verifica-se que a magnitude da tensão máxima não excede largamente o valor da tensão limite normalizada, de 1 . Dentro do intervalo $\eta \in[0.25,0.75]$, as tensões associadas ao modelo baseado em pixel excedem a restrição de tensão em apenas $4.7 \%$.

Analisando as tensões máximas provenientes dos modelos ajustados ao corpo, maiores diferenças são observadas. Conforme ilustrado na Figura 33, para $N_{e}=25600$, a curva de nível obtida para $\eta=0.25$ apresenta um pequeno canto reentrante. Neste caso, as respostas obtidas tanto com o modelo baseado em pixel quanto com o modelo ajustado ao corpo são questionáveis, pois não se pode garantir convergência de tensão com refino de malha em tal situação, devido a presença de canto de singularidade de tensão no modelo 
físico.

Para $\eta>0.25$, as curvas de nível apresentam comportamento muito mais suave na região do canto reentrante, permitindo melhores comparações entre ambos os modelos. Dentro do intervalo $\eta \in[0.3,0.75]$, as tensões provenientes dos modelos ajustados ao corpo excedem a restrição de tensão em até $12.2 \%$.

Analisando estes resultados, verifica-se que apesar de boa resposta em tensão ser obtida com uma malha mais grosseira, uma malha mais refinada é desejável para melhor descrever a resposta em tensão das estruturas suaves subjacentes. Na Figura 33, topologias idênticas são observadas; no entanto, pequenas diferenças na forma das estruturas suaves subjacentes parecem ser cruciais quando o objetivo é obter uma boa resposta em tensão. Além disso, uma malha mais refinada parece melhor representar o efeito de singularidade de tensão do canto reentrante, como pode ser visto na Figura 33, onde a curva de nível para $\eta=0.25$ encosta no canto reentrante para a malha mais grosseira, mas é absolutamente suave para a malha mais refinada.

\subsubsection{Resultados numéricos complementares}

De forma a complementar a seção de resultados, dois problemas adicionais são solucionados. Os resultados obtidos são pós-processados com modelos ajustados ao corpo, utilizando o mesmo esquema de pós-processamento apresentado anteriormente. Os dados de entrada para o algoritmo de otimização e as propriedades do material são os mesmos considerados na subseção 7.2 , a menos que especificado de outra forma.

\subsubsection{Problema de domínio com furo circular}

O problema de domínio com furo circular aqui abordado (AMSTUTZ; NOVOTNY, 2010; PEREIRA; FANCELLO; BARCELLOS, 2004), Figura 35, é discretizado com uma malha distorcida contendo 52608 elementos bilineares isoparamétricos de quatro nós. $\mathrm{O}$ problema é solucionado considerando as condições de simetria, seguindo Pereira, Fancello e Barcellos (2004). A força vertical é distribuída sobre a parte superior do furo circular $\left(x_{2} \geqslant 0\right)$ de acordo com a função $f\left(x_{1}\right)=-x_{1}^{2}+1.5^{2}$, onde $\left(x_{1}, x_{2}\right)=(0,0)$ é o centroide do furo.

Dados de entrada: raio do filtro $R=0.5$ e tensão de escoamento $\sigma_{y}=5 \times 10^{3}$. Os valores de $\eta$ (projeção parametrizada) são escolhidos como $\eta_{d}=0.4, \eta_{i}=0.5 \mathrm{e}$ $\eta_{e}=0.6$. Neste exemplo, como uma malha não regular é utilizada para discretizar o domínio de projeto, o valor de $\delta_{\text {lim }}$ é calculado para o maior elemento da malha, como $\delta_{\text {lim }}=2 \times R / l_{e}=2 \times 0.5 / 0.065=15.4$. O valor de $\delta$ é atualizado considerando uma abordagem de três passos, até o valor máximo de $\delta_{\max }$, como: $\delta^{(1)}=1, \delta^{(2)}=4$ e $\delta^{(3)}=8$, com $\delta_{\text {max }} \cong \delta_{\text {lim }} / 2$. A solução otimizada é obtida em 515 iterações. 
Figura 35: Problema de domínio com furo circular.
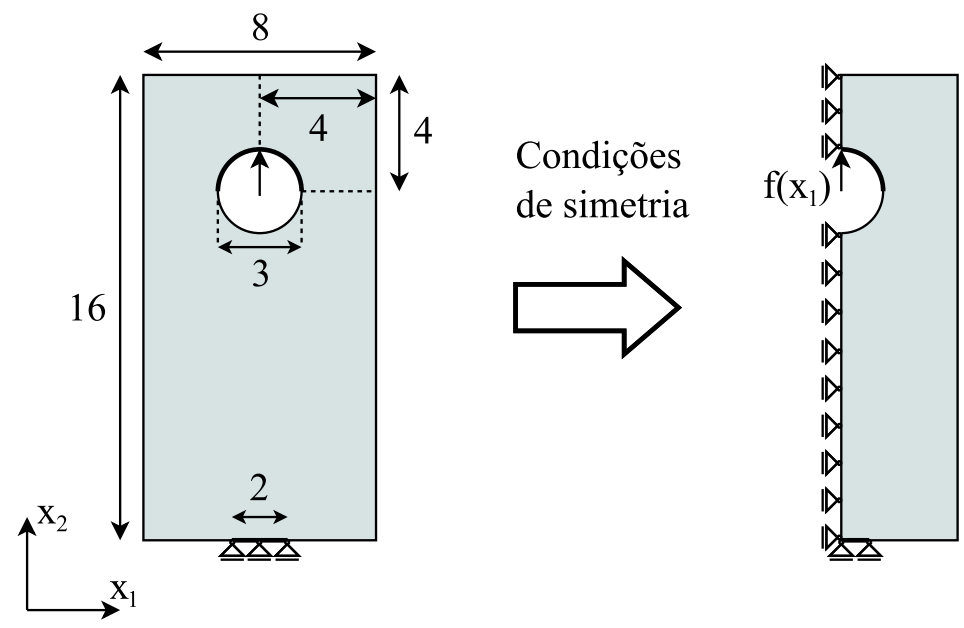

Fonte: adaptado de Silva, Beck e Sigmund (2019).

A Figura 36 mostra as topologias erodida, intermediária e dilatada obtidas como resposta do problema de otimização, tal como as suas respectivas curvas de nível, tensões baseadas em pixel e tensões ajustadas ao corpo. A Figura 37 mostra os gráficos de pósprocessamento, onde as tensões equivalentes de von Mises máximas são calculadas para os modelos baseados em pixel e ajustados ao corpo, dentro do intervalo $\eta \in[0.4,0.6]$, ou seja, o intervalo considerado na otimização topológica.

Analisando a Figura 36, observa-se que as topologias erodida, intermediária e dilatada são iguais. Em relação às distribuições de tensão, apesar da estrutura erodida ser a mais solicitada, verifica-se que as estruturas intermediária e dilatada também apresentam áreas altamente solicitadas, perto da região onde a força distribuída termina.

As tensões baseadas em pixel e ajustadas ao corpo, Figura 36, mostram uma região com baixas tensões de von Mises próxima do contorno onde a força distribuída é aplicada. As tensões de von Mises são pequenas nestes pontos, pois estes são pontos de transição onde a tensão passa de compressão para tração. Estes resultados podem ser utilizados para traçar alguns paralelos com o trabalho de Amstutz e Novotny (2010), onde a solução deste mesmo problema apresenta pequenos furos nestas regiões, indicando que o volume estrutural pode ser ainda mais minimizado ao remover material destas regiões pouco estressadas; entretanto ao custo de se obter estruturas sensíveis a variações uniformes de manufatura. As soluções otimizadas ilustradas na Figura 36 não apresentam os pequenos furos na região onde a força é aplicada, pois a formulação robusta de três campos é empregada para solucionar o problema. Conforme apresentado em Wang, Lazarov e Sigmund (2011), a formulação robusta garante uma escala de comprimento mínimo tanto na fase sólida quanto na fase vazia da topologia. Neste exemplo, o aparecimento destes pequenos furos é suprimido pelo uso de um grande raio de filtragem.

O gráfico de pós-processamento, Figura 37, indica que uma estrutura verdadeira- 
Figura 36: Topologias (esquerda) e respectivas curvas de nível (direita). Tensões obtidas com modelos baseados em pixel (esquerda) e ajustados ao corpo (direita). Problema de domínio com furo circular.
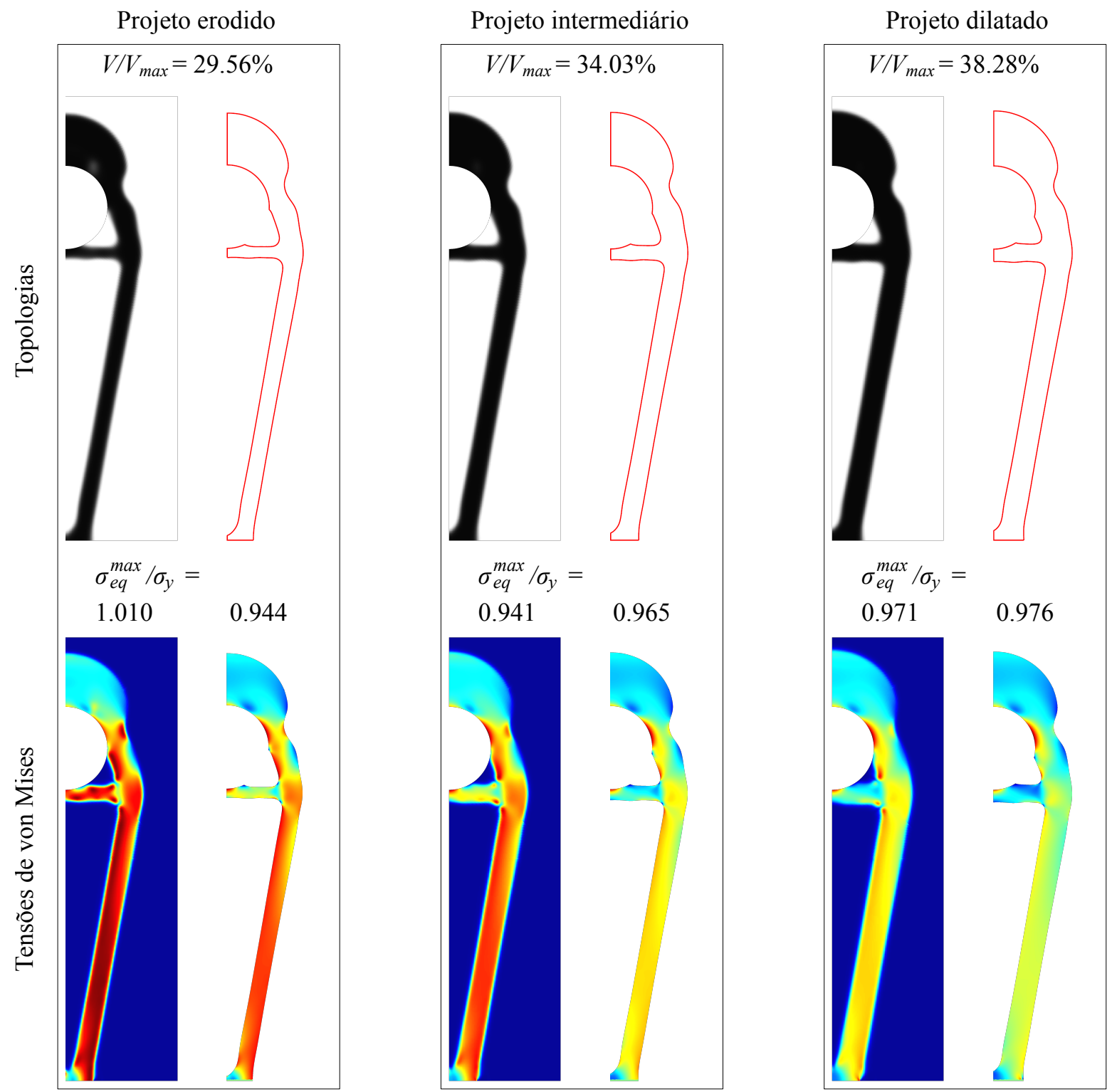

Fonte: adaptado de Silva, Beck e Sigmund (2019).

mente robusta é obtida, pois todos os projetos dentro do intervalo $\eta \in[0.4,0.6]$ apresentam uma tensão de von Mises máxima que satisfaz a restrição de tensão considerando a tolerância prescrita de $t o l_{\sigma}=1 \%$, tanto para os modelos baseados em pixel quanto para os modelos ajustados ao corpo. Além disso, a tensão de von Mises máxima apresenta um comportamento suave para $\eta \in[0.4,0.6]$.

\subsubsection{Problema de domínio com corte triangular}

O problema de domínio com corte triangular (LE et al., 2010; LIAN et al., 2017), Figura 38, é discretizado com uma malha regular com 125538 elementos, correspondente a uma grade de $600 \times 300$ elementos, desconsiderando os elementos que possuírem nós 
Figura 37: Tensões de von Mises máximas pós-processadas, problema de domínio com furo circular. Resultados referentes às estruturas erodida, intermediária e dilatada, considerando o modelo ajustado ao corpo, são indicados por círculos.

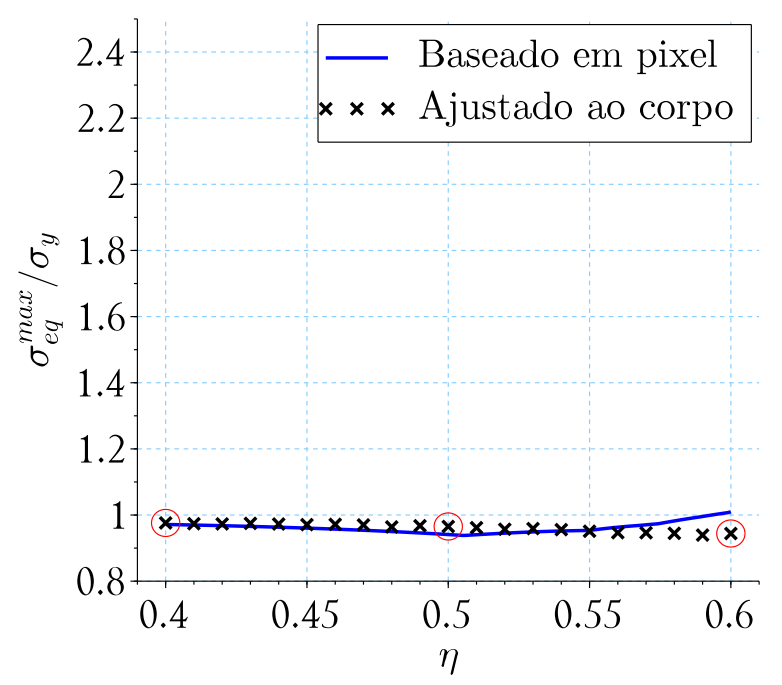

Fonte: adaptado de Silva, Beck e Sigmund (2019).

dentro da região do corte triangular, os quais são removidos da malha.

Figura 38: Problema de domínio com corte triangular.

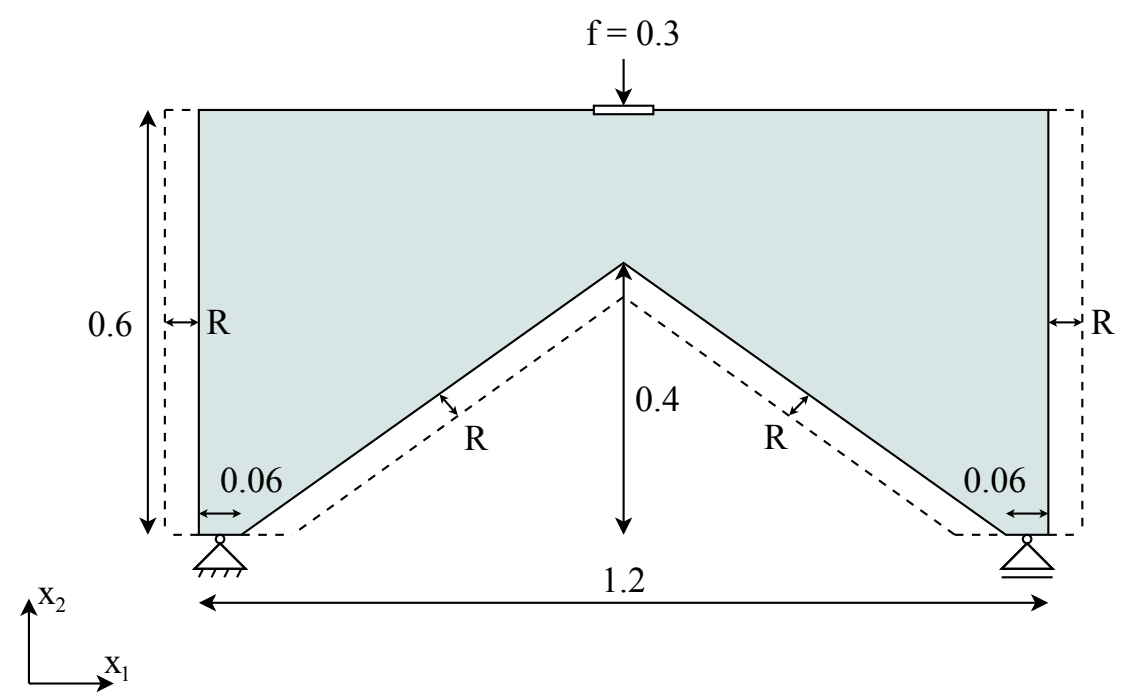

Fonte: Silva, Beck e Sigmund (2019).

Dados de entrada: raio do filtro $R=0.016$, com extensão de domínio de filtragem conforme ilustrado na Figura 38, e tensão de escoamento $\sigma_{y}=16 \times 10^{3}$. Os valores de $\eta$ são escolhidos como $\eta_{d}=0.25, \eta_{i}=0.5$ e $\eta_{e}=0.75$. Neste exemplo, $\delta_{\text {lim }}=2 \times R / l_{e}=$ $2 \times 0.016 /(1.2 / 600)=16$. O valor de $\delta$ é atualizado considerando uma abordagem de três passos, até o valor máximo de $\delta_{\max }$, como: $\delta^{(1)}=1, \delta^{(2)}=4$ e $\delta^{(3)}=8$, com $\delta_{\max }=\delta_{\text {lim }} / 2$. A força aplicada, de 0.3, é distribuída sobre o comprimento de 0.08 , para evitar concentrações de tensão. As tensões de von Mises próximas às regiões de apoio não são consideradas 
durante as etapas de otimização e pós-processamento. A solução otimizada é obtida em 897 iterações.

A Figura 39 mostra as topologias erodida, intermediária e dilatada obtidas como resposta do problema de otimização, tal como as suas respectivas curvas de nível, tensões baseadas em pixel e tensões ajustadas ao corpo. Os gráficos de pós-processamento são mostrados na Figura 40, dentro do intervalo $\eta \in[0.25,0.75]$.

Figura 39: Topologias (linha 1), respectivas curvas de nível (linha 2), tensões obtidas com modelos baseados em pixel (linha 3) e ajustados ao corpo (linha 4). Problema de domínio com corte triangular.
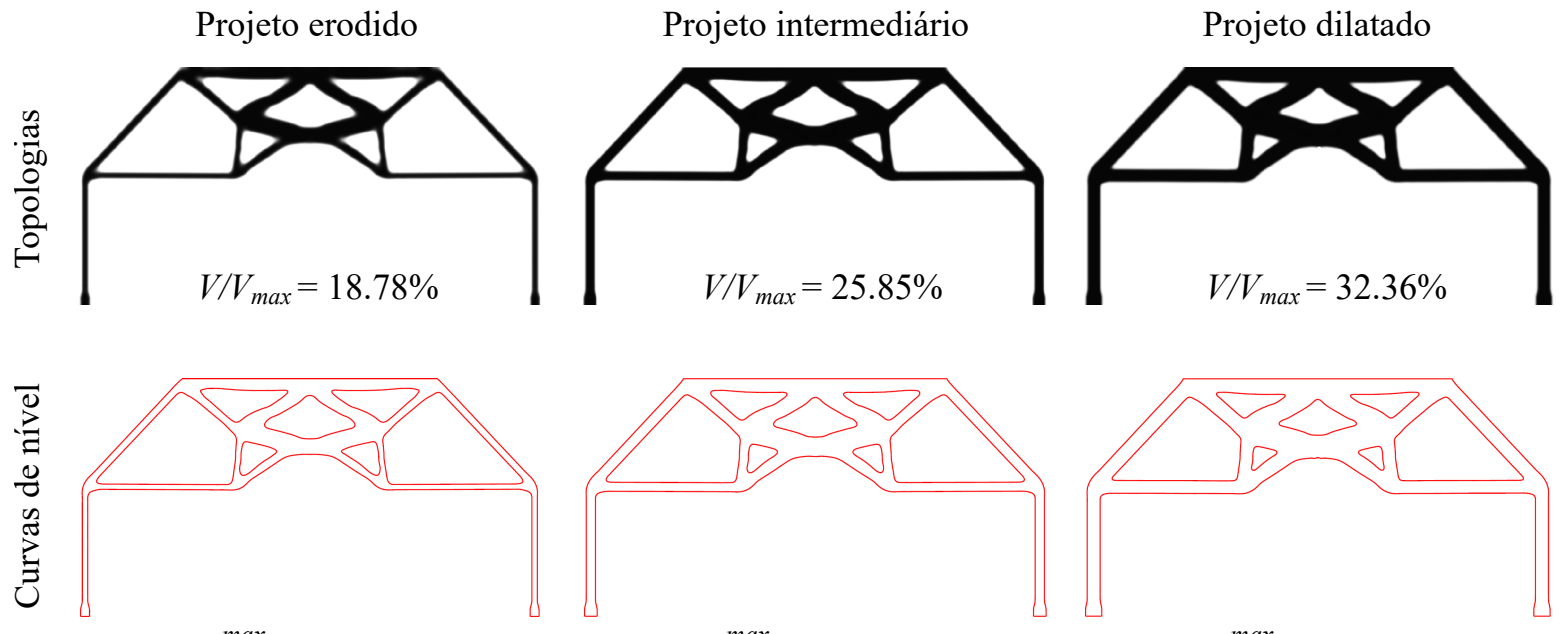

$\sigma_{e q}^{\max } / \sigma_{y}=1.010$

$\sigma_{e q}^{\max } / \sigma_{y}=0.979$
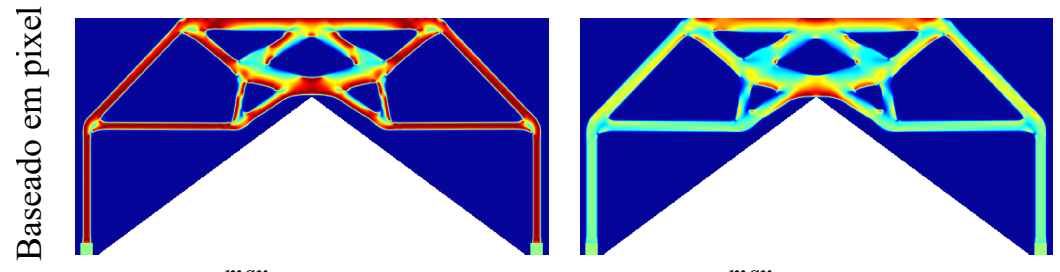

$\sigma_{e q}^{\max } / \sigma_{y}=0.951$
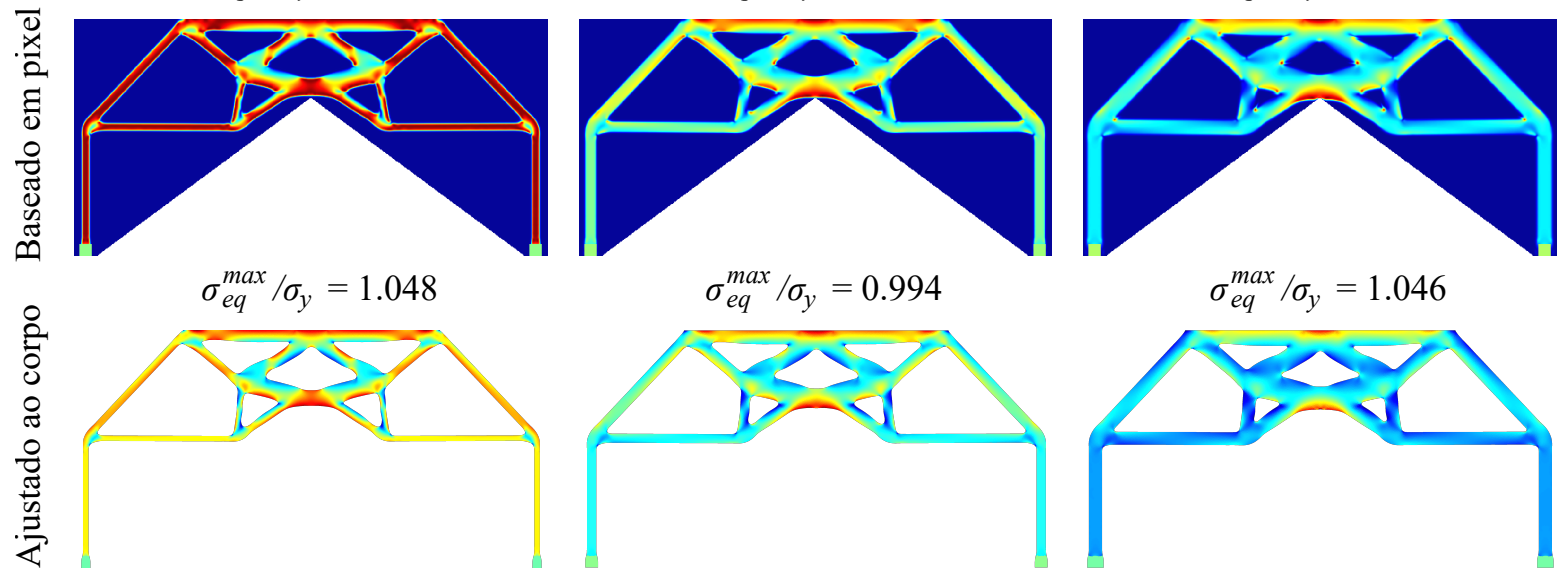

Fonte: adaptado de Silva, Beck e Sigmund (2019).

Analisando a Figura 39, verifica-se que as topologias erodida, intermediária e dilatada são as mesmas. Todas as estruturas evitaram adequadamente o canto de singularidade de tensão proveniente do corte triangular, no sentido em que as tensões de von Mises satisfazem localmente a restrição de tensão nestas regiões. Apesar da estrutura erodida ser a mais solicitada, ambas as estruturas intermediária e dilatada apresentam regiões altamente solicitadas, perto do canto reentrante no domínio de projeto e perto da força distribuída aplicada. Além disso, verifica-se que as tensões ajustadas ao corpo estão em concordância com as tensões baseadas em pixel. 
Figura 40: Tensões de von Mises máximas pós-processadas, problema de domínio com corte triangular. Resultados referentes às estruturas erodida, intermediária e dilatada, considerando o modelo ajustado ao corpo, são indicados por círculos.

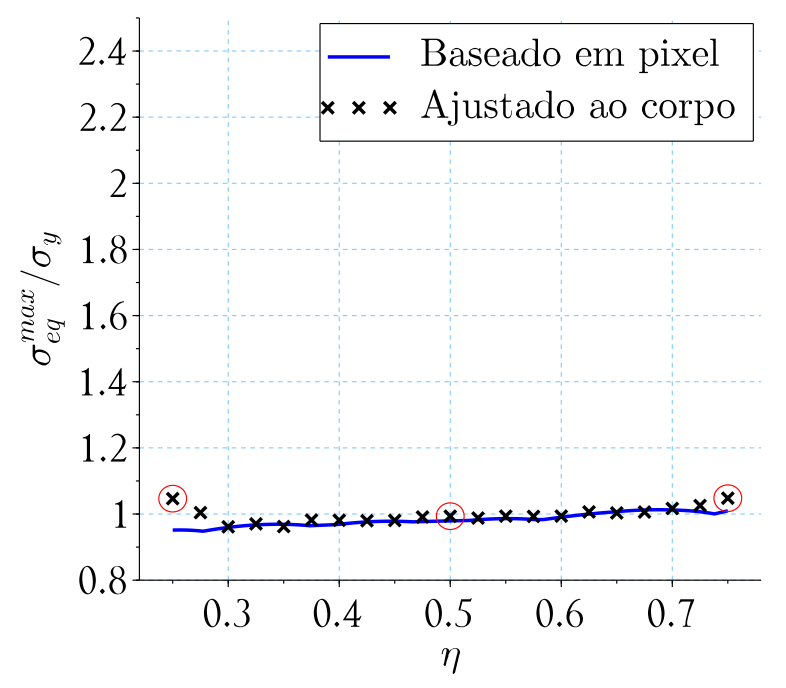

Fonte: adaptado de Silva, Beck e Sigmund (2019).

O gráfico de pós-processamento, Figura 40, mostra que a tensão de von Mises máxima possui um comportamento suave em todo o intervalo $\eta \in[0.25,0.75]$. As tensões provenientes do modelo baseado em pixel excedem a restrição de tensão em, no máximo, $1.3 \%$, enquanto que este valor é de $4.8 \%$ para o modelo ajustado ao corpo, indicando que a solução obtida é verdadeiramente robusta em relação a variações uniformes de contorno. 



\section{CONCLUSÕES}

Este trabalho aborda o problema de otimização topológica de estruturas contínuas com restrição de tensão sob incertezas. Especificamente, quatro formulações foram propostas para lidar com incertezas no problema de minimização de volume com restrição de tensão. Três delas para lidar com incertezas na intensidade e direção das forças aplicadas: 1) formulação robusta probabilística, onde cada restrição de tensão é escrita como uma soma ponderada entre o seu valor esperado e o seu desvio padrão, os quais foram obtidos com uma abordagem de perturbação de primeira ordem; 2) formulação baseada em confiabilidade, onde consideram-se restrições probabilísticas sobre cada ponto de cálculo de tensão, por meio de uma abordagem acoplada de primeira ordem; e 3) formulação robusta não probabilística, onde considera-se o pior cenário para as restrições de tensão, por meio de uma abordagem acoplada de otimização com anti-otimização. A quarta e última formulação foi proposta para lidar com incerteza uniforme de manufatura: 4) formulação robusta não probabilística de três campos, onde três topologias são consideradas simultaneamente no procedimento de otimização, de forma a simular possíveis erros de manufatura.

A abordagem baseada em densidade foi empregada na formulação dos problemas de otimização topológica. Os problemas resultantes, que apresentam um elevado número de restrições de tensão, foram solucionados com uma abordagem proposta neste trabalho, baseada no método do Lagrangiano aumentado: 1) todas as restrições de tensão são incluídas na função objetivo, ponderadas por um parâmetro de penalização e multiplicadores de Lagrange; 2) os subproblemas resultantes são solucionados com um método de máxima descida, modificado para lidar com as restrições laterais; 3) uma abordagem de continuação é utilizada para atualizar o parâmetro de projeção e obter topologias 0/1. A abordagem de solução proposta é genérica e pode ser utilizada para solucionar qualquer problema de otimização topológica com restrição de tensão, sendo ele determinístico ou sob incertezas.

Diversos problemas foram solucionados para mostrar a aplicabilidade das formulações propostas. Os resultados obtidos mostraram que as topologias resultantes da abordagem determinística são extremamente sensíveis a incertezas. Por outro lado, as formulações não determinísticas se mostraram válidas alternativas a formulação tradicional determinística, fornecendo resultados verdadeiramente confiáveis e robustos.

\subsection{Conclusões específicas: problemas sob incerteza nas forças}

Considerando os problemas sob incertezas nas forças aplicadas, apesar de abordagens de primeira ordem serem empregadas nas formulações probabilísticas, boa concordância foi obtida no pós-processamento com o método de Simulação de Monte Carlo. No problema analisado sujeito a duas forças não correlacionadas de intensidade Gaussiana, as abordagens 
robusta probabilística e baseada em confiabilidade forneceram resultados quase idênticos, demonstrando que a abordagem robusta, neste caso, pode ser utilizada como uma alternativa a abordagem baseada em confiabilidade. Mostrou-se, também, que a abordagem não probabilística fornece um resultado mais conservador que as demais abordagens, pois esta leva em conta a combinação de extremos dos intervalos, garantindo a viabilidade de tensão também para estes casos, ao contrário do que acontece nas abordagens probabilísticas, onde as combinações de extremos são negligenciadas devido às suas baixas probabilidades de ocorrência. Quando uma única força de intensidade incerta foi considerada, as três abordagens não determinísticas forneceram resultados equivalentes. Isto é justificado, no caso da abordagem robusta não probabilística, uma vez que não existe uma associação de extremos quando uma única variável incerta é considerada.

Os problemas sob incerteza na direção das forças aplicadas foram solucionados com a abordagem robusta não probabilística. Mostrou-se, neste caso, que um resultado verdadeiramente robusto pode ser obtido com a abordagem proposta, e que a abordagem determinística sob vários casos de carga não é capaz de garantir a robustez da estrutura otimizada.

As formulações sob incerteza nas forças aplicadas se mostraram muito não lineares, exigindo um maior número de iterações para satisfazer os critérios de convergência impostos pelo projetista, quando comparadas a abordagem tradicional determinística.

\subsection{Conclusões específicas: problemas sob incerteza de manufatura}

Considerando os problemas sob incerteza de manufatura, solucionados com a abordagem robusta de três campos proposta, boa concordância foi obtida no pós-processamento baseado em modelos de elementos finitos ajustados ao corpo. Foi demonstrado que a abordagem proposta é capaz de obter topologias tolerantes a erros de manufatura e que

satisfazem as restrições de tensão. Um estudo de dependência da malha foi realizado sobre o problema em forma de L, demonstrando que topologias idênticas são obtidas para dois tamanhos de malha distintos, com pequenas diferenças em suas formas. Entretanto, a solução otimizada considerando a malha mais refinada apresentou maior robustez em relação a incertezas de manufatura, demonstrando que estas pequenas diferenças na forma da topologia otimizada são cruciais para garantir acurácia de tensão e, consequentemente, robustez da topologia suave subjacente.

\subsection{Sugestões para trabalhos futuros}

Esta subseção apresenta algumas sugestões para trabalhos futuros, relacionadas ao problema de otimização topológica com restrição de tensão sob incertezas:

- Estender a formulação robusta probabilística para problemas com comportamento 
não linear geométrico;

- Estender a formulação robusta de três campos para problemas com comportamento não linear geométrico;

- Estender as formulações propostas para problemas de mecanismos flexíveis com restrição de tensão;

- Propor uma formulação unificada, para lidar de forma simultânea com incertezas de manufatura e de forças externas. Esta formulação pode ser obtida por meio da extensão da formulação apresentada na Equação (5.20), considerando que cada um dos três campos de densidades relativas está sujeito a incertezas nas forças externas. Desta forma, obtém-se um problema de otimização com três restrições probabilísticas (ou não probabilísticas, considerando a abordagem de anti-otimização) por ponto de cálculo de tensão;

- Propor uma formulação para lidar com incerteza na posição das forças aplicadas;

- Propor uma formulação baseada em confiabilidade considerando a probabilidade de falha do sistema, em vez de restrições probabilísticas sobre cada ponto de cálculo de tensão;

- Estudar alternativas ao método utilizado na solução dos subproblemas de otimização, originados do método do Lagrangiano aumentado;

- Estudar alternativas a abordagem utilizada para garantir acurácia no cálculo de tensão nas interfaces irregulares, entre as fases sólida e vazia da topologia. 



\section{REFERÊNCIAS}

ALLAIRE, G.; JOUVE, F.; TOADER, A.-M. Structural optimization using sensitivity analysis and a level-set method. Journal of Computational Physics, v. 194, n. 1, p. 363-393, 2004.

ALLEN, M.; MAUTE, K. Reliability-based shape optimization of structures undergoing fluid-structure interaction phenomena. Computer Methods in Applied Mechanics and Engineering, v. 194, n. 30-33, p. 3472-3495, 2005.

AMBROSIO, L.; BUTTAZZO, G. An optimal design problem with perimeter penalization. Calculus of Variations and Partial Differential Equations, v. 1, n. 1, p. 55-69, 1993.

AMSTUTZ, S.; NOVOTNY, A. A. Topological optimization of structures subject to von mises stress constraints. Structural and Multidisciplinary Optimization, v. 41, n. 3, p. 407-420, 2010.

AMSTUTZ, S.; NOVOTNY, A. A.; NETO, E. A. de S. Topological derivative-based topology optimization of structures subject to drucker-prager stress constraints.

Computer Methods in Applied Mechanics and Engineering, v. 233-236, p. 123-136, 2012.

AOUES, Y.; CHATEAUNEUF, A. Reliability-based optimization of structural systems by adaptive target safety - application to rc frames. Structural Safety, v. 30, n. 2, p. 144-161, 2008.

AOUES, Y.; CHATEAUNEUF, A. Benchmark study of numerical methods for reliabilitybased design optimization. Structural and Multidisciplinary Optimization, v. 41, n. 2, p. 277-294, 2010.

ARORA, J. S. Introduction to Optimum Design. 3rd. ed. Boston: Academic Press, 2012 .

ASADPOURE, A.; TOOTKABONI, M.; GUEST, J. K. Robust topology optimization of structures with uncertainties in stiffness - application to truss structures. Computers \& Structures, v. 89, n. 11-12, p. 1131-1141, 2011.

BA-ABBAD, M. A.; NIKOLAIDIS, E.; KAPANIA, R. K. New approach for system reliability-based design optimization. AIAA Journal, v. 44, n. 5, p. 1087-1096, 2006.

BARLOW, J. Optimal stress locations in finite element models. International Journal for Numerical Methods in Engineering, v. 10, n. 2, p. 243-251, 1976.

BATHE, K.-J. Finite Element Procedures. Upper Sadle River, New Jersey: Prentice Hall, 1996.

BECK, A. T.; GOMES, W. J. S. A comparison of deterministic, reliability-based and risk-based structural optimization under uncertainty. Probabilistic Engineering Mechanics, v. 28, p. 18-29, 2012. 
BECK, A. T. et al. A comparison between robust and risk-based optimization under uncertainty. Structural and Multidisciplinary Optimization, v. 52, n. 3, p. 479-492, 2015.

BEN-TAL, A.; NEMIROVSKI, A. Robust optimization - methodology and applications. Mathematical Programming, v. 92, n. 3, p. 453-480, 2002.

BENDSØE, M. P.; KIKUCHI, N. Generating optimal topologies in structural design using a homogenization method. Computer Methods in Applied Mechanics and Engineering, v. 71, n. 2, p. 197-224, 1988.

BENDSØE, M. P.; SIGMUND, O. Material interpolation schemes in topology optimization. Archive of Applied Mechanics, v. 69, n. 9-10, p. 635-654, 1999.

BENDSØE, M. P.; SIGMUND, O. Topology Optimization: Theory, Methods and Applications. Berlin: Springer, 2003.

BEYER, H.-G.; SENDHOFF, B. Robust optimization - a comprehensive survey. Computer Methods in Applied Mechanics and Engineering, v. 196, n. 33-34, p. 3190-3218, 2007.

BIRGIN, E. G.; MARTÍnEZ, J. M. Practical Augmented Lagrangian Methods for Constrained Optimization. Philadelphia, PA: Society for Industrial and Applied Mathematics, 2014.

BRUGGI, M. On an alternative approach to stress constraints relaxation in topology optimization. Structural and Multidisciplinary Optimization, v. 36, n. 2, p. 125-141, 2008.

BRUGGI, M.; DUYSINX, P. Topology optimization for minimum weight with compliance and stress constraints. Structural and Multidisciplinary Optimization, v. 46, n. 3, p. 369-384, 2012.

BRUNS, T. E.; TORTORELLI, D. A. Topology optimization of non-linear elastic structures and compliant mechanisms. Computer Methods in Applied Mechanics and Engineering, v. 190, n. 26-27, p. 3443-3459, 2001.

CARDOSO, E. L.; FONSECA, J. S. O. Complexity control in the topology optimization of continuum structures. Journal of the Brazilian Society of Mechanical Sciences and Engineering, v. 25, n. 3, p. 293-301, 2003.

CHARRAS, G. T.; GULDBERG, R. E. Improving the local solution accuracy of large-scale digital image-based finite element analyses. Journal of Biomechanics, v. 33 , n. 2, p. 255-259, 2000.

CHENG, G. D.; GUO, X. $\varepsilon$-relaxed approach in structural topology optimization. Structural Optimization, v. 13, n. 4, p. 258-266, 1997.

CHENG, K.-T.; OLHOFF, N. An investigation concerning optimal design of solid elastic plates. International Journal of Solids and Structures, v. 17, n. 3, p. 305-323, 1981.

CHO, K.-H. et al. Reliability-based topology optimization based on bidirectional evolutionary structural optimization using multi-objective sensitivity numbers.

International Journal of Automotive Technology, v. 12, n. 6, p. 849-856, 2011. 
CLAUSEN, A.; ANDREASSEN, E. On filter boundary conditions in topology optimization. Structural and Multidisciplinary Optimization, v. 56, n. 5, p. 1147-1155, 2017.

DÍAZ, A.; SIGMUND, O. Checkerboard patterns in layout optimization. Structural optimization, v. 10, n. 1, p. 40-45, 1995.

DITLEVSEN, O.; MADSEN, H. O. Structural Reliability Methods. Internet Edition, 2007. Disponível em: <http://od-website.dk/books/OD-HOM-StrucRelMeth-Ed2.3.7. $\mathrm{pdf}>$.

DOITRAND, A. et al. Comparison between voxel and consistent meso-scale models of woven composites. Composites Part A: Applied Science and Manufacturing, v. 73, p. 143-154, 2015.

DU, X.; CHEN, W. Sequential optimization and reliability assessment method for efficient probabilistic design. Journal of Mechanical Design, v. 126, n. 2, p. 225-233, 2004.

DUYSINX, P.; BENDSØE, M. P. Topology optimization of continuum structures with local stress constraints. International Journal for Numerical Methods in Engineering, v. 43, n. 8, p. 1453-1478, 1998.

DUYSINX, P.; BRUYNEEL, M.; FLEURY, C. Solution of large scale optimization problems with sequential convex programming. Liège, Belgium, 2009. Disponível em: <https://orbi.uliege.be/bitstream/2268/25613/1/Duysinx_DCAMM09.pdf>.

DUYSINX, P.; SIGMUND, O. New developments in handling stress constraints in optimal material distribution. In: 7th AIAA/USAF/NASA/ISSMO Symposium on Multidisciplinary Analysis and Optimization. St. Louis, United States: Multidisciplinary Analysis Optimization Conferences, 1998. p. 1501-1509.

ELISHAKOFF, I. Probabilistic Theory of Structures. 2nd. ed. London: Dover, 1999.

ELISHAKOFF, I.; HAFTKA, R. T.; FANG, J. Structural design under bounded uncertainty - optimization with anti-optimization. Computers \& Structures, v. 53, n. 6, p. 1401-1405, 1994.

ELISHAKOFF, I.; OHSAKI, M. Optimization and Anti-Optimization of Structures Under Uncertainty. London: Imperial College Press, 2010.

EMMENDOERFER, H.; FANCELLO, E. A. A level set approach for topology optimization with local stress constraints. International Journal for Numerical Methods in Engineering, v. 99, n. 2, p. 129-156, 2014.

EMMENDOERFER, H.; FANCELLO, E. A. Topology optimization with local stress constraint based on level set evolution via reaction-diffusion. Computer Methods in Applied Mechanics and Engineering, v. 305, p. 62-88, 2016.

EMMENDOERFER, H.; SILVA, E. C. N.; FANCELLO, E. A. Stress-constrained level set topology optimization for design-dependent pressure load problems. Computer Methods in Applied Mechanics and Engineering, v. 344, p. 569-601, 2019.

FANCELLO, E. A. Topology optimization for minimum mass design considering local failure constraints and contact boundary conditions. Structural and Multidisciplinary Optimization, v. 32, n. 3, p. 229-240, 2006. 
FANCELLO, E. A.; PEREIRA, J. T. Structural topology optimization considering material failure constraints and multiple load conditions. Latin American Journal of Solids and Structures, v. 1, n. 1, p. 3-24, 2003.

FANG, G. et al. Smoothing artificial stress concentrations in voxel-based models of textile composites. Composites Part A: Applied Science and Manufacturing, v. 80, p. 270-284, 2016.

FLEURY, C. Conlin: An efficient dual optimizer based on convex approximation concepts. Structural optimization, v. 1, n. 2, p. 81-89, 1989.

GEUZAINE, C.; REMACLE, J.-F. Gmsh: A 3-d finite element mesh generator with built-in pre- and post-processing facilities. International Journal for Numerical Methods in Engineering, v. 79, n. 11, p. 1309-1331, 2009.

GUEST, J. K.; PRÉVOST, J. H.; BELYTSCHKO, T. Achieving minimum length scale in topology optimization using nodal design variables and projection functions. International Journal for Numerical Methods in Engineering, v. 61, n. 2, p. 238-254, 2004.

GULDBERG, R. E.; HOLLISTER, S. J.; CHARRAS, G. T. The accuracy of digital image-based finite element models. Journal of Biomechanical Engineering, v. 120, n. 2, p. 289-295, 1998.

GUO, X. et al. Confidence structural robust design and optimization under stiffness and load uncertainties. Computer Methods in Applied Mechanics and Engineering, v. 198, n. 41-44, p. 3378-3399, 2009.

GURAV, S. P.; GOOSEN, J. F. L.; VANKEULEN, F. Bounded-but-unknown uncertainty optimization using design sensitivities and parallel computing: Application to mems.

Computers \& Structures, v. 83, n. 14, p. 1134-1149, 2005.

HOLLISTER, S. J.; RIEMER, B. A. Digital-image-based finite element analysis for bone microstructure using conjugate gradient and gaussian filter techniques. In: Proceedings SPIE. San Diego, United States: Mathematical Methods in Medical Imaging II, 1993. v. 2035, p. 95-106.

HOLMBERG, E.; THORE, C.-J.; KLARBRING, A. Game theory approach to robust topology optimization with uncertain loading. Structural and Multidisciplinary Optimization, v. 55, n. 4, p. 1383-1397, 2017.

HOLMBERG, E.; TORSTENFELT, B.; KLARBRING, A. Stress constrained topology optimization. Structural and Multidisciplinary Optimization, v. 48, n. 1, p. 33-47, 2013.

JOG, C. S.; HABER, R. B. Stability of finite element models for distributed-parameter optimization and topology design. Computer Methods in Applied Mechanics and Engineering, v. 130, n. 3-4, p. 203-226, 1996.

JUNG, H.-S.; CHO, S. Reliability-based topology optimization of geometrically nonlinear structures with loading and material uncertainties. Finite Elements in Analysis and Design, v. 41, n. 3, p. 311-331, 2004. 
KANG, Z.; LIU, P. Reliability-based topology optimization against geometric imperfections with random threshold model. International Journal for Numerical Methods in Engineering, v. 115, n. 1, p. 99-116, 2018.

KIYONO, C. Y.; SILVA, E. C. N.; REDDY, J. N. Optimal design of laminated piezocomposite energy harvesting devices considering stress constraints. International Journal for Numerical Methods in Engineering, v. 105, n. 12, p. 883-914, 2016.

KIYONO, C. Y. et al. A new multi-p-norm formulation approach for stress-based topology optimization design. Composite Structures, v. 156, p. 10-19, 2016.

KOGISO, N. et al. Robust topology optimization for compliant mechanisms considering uncertainty of applied loads. Journal of Advanced Mechanical Design, Systems, and Manufacturing, v. 2, n. 1, p. 96-107, 2008.

KOHN, R. V.; STRANG, G. Optimal design and relaxation of variational problems, i. Communications on Pure and Applied Mathematics, v. 39, n. 1, p. 113-137, 1986.

KOHN, R. V.; STRANG, G. Optimal design and relaxation of variational problems, ii. Communications on Pure and Applied Mathematics, v. 39, n. 2, p. 139-182, 1986.

KOHN, R. V.; STRANG, G. Optimal design and relaxation of variational problems, iii. Communications on Pure and Applied Mathematics, v. 39, n. 3, p. 353-377, 1986.

LAMBERTI, L.; PAPPALETTERE, C. Comparison of the numerical efficiency of different sequential linear programming based algorithms for structural optimisation problems.

Computers \& Structures, v. 76, n. 6, p. 713-728, 2000.

LAZAROV, B. S.; SCHEVENELS, M.; SIGMUND, O. Topology optimization with geometric uncertainties by perturbation techniques. International Journal for Numerical Methods in Engineering, v. 90, n. 11, p. 1321-1336, 2012.

LE, C. et al. Stress-based topology optimization for continua. Structural and Multidisciplinary Optimization, v. 41, n. 4, p. 605-620, 2010.

LEON, D. M. de et al. Stress-constrained topology optimization for compliant mechanism design. Structural and Multidisciplinary Optimization, v. 52, n. 5, p. 929-943, 2015 .

LIAN, H. et al. Combined shape and topology optimization for minimization of maximal von mises stress. Structural and Multidisciplinary Optimization, v. 55, n. 5, p. 1541-1557, 2017.

LIANG, J.; MOURELATOS, Z. P.; NIKOLAIDIS, E. A single-loop approach for system reliability-based design optimization. Journal of Mechanical Design, v. 129, n. 12, p. 1215-1224, 2007.

LOMBARDI, M. Optimization of uncertain structures using non-probabilistic models. Computers \& Structures, v. 67, n. 1-3, p. 99-103, 1998.

LOMBARDI, M.; HAFTKA, R. T. Anti-optimization technique for structural design under load uncertainties. Computer Methods in Applied Mechanics and Engineering, v. 157, n. 1-2, p. 19-31, 1998. 
LONG, K.; WANG, X.; LIU, H. Stress-constrained topology optimization of continuum structures subjected to harmonic force excitation using sequential quadratic programming. Structural and Multidisciplinary Optimization, 2018.

LOPES, C. G.; NOVOTNY, A. A. Topology design of compliant mechanisms with stress constraints based on the topological derivative concept. Structural and Multidisciplinary Optimization, v. 54, n. 4, p. 737-746, 2016.

LOPEZ, R. H.; BECK, A. T. Reliability-based design optimization strategies based on form: a review. Journal of the Brazilian Society of Mechanical Sciences and Engineering, v. 34, n. 4, p. 506-514, 2012.

LUO, Y.; WANG, M. Y.; KANG, Z. An enhanced aggregation method for topology optimization with local stress constraints. Computer Methods in Applied Mechanics and Engineering, v. 254, p. 31-41, 2013.

LUO, Y. et al. Reliability based topology optimization for continuum structures with local failure constraints. Computers \& Structures, v. 143, p. 73-84, 2014.

MAUTE, K.; FRANGOPOL, D. M. Reliability-based design of mems mechanisms by topology optimization. Computers \& Structures, v. 81, n. 8-11, p. 813-824, 2003.

MELCHERS, R. E.; BECK, A. T. Structural Reliability Analysis and Prediction. 3rd. ed. New Jersey: Wiley, 2018.

MIGUEL, L. F. F. et al. Robust design optimization of tmds in vehicle-bridge coupled vibration problems. Engineering Structures, v. 126, p. 703-711, 2016.

NGUYEN, T. H.; SONG, J.; PAULINO, G. H. Single-loop system reliability-based design optimization using matrix-based system reliability method: Theory and applications.

Journal of Mechanical Design, v. 132, n. 1, p. 011005-1-11, 2009.

PARÍS, J. et al. Topology optimization of continuum structures with local and global stress constraints. Structural and Multidisciplinary Optimization, v. 39, n. 4, p. 419-437, 2009.

PARK, G.-J. et al. Robust design: An overview. AIAA Journal, v. 44, n. 1, p. 181-191, 2006.

PEREIRA, A. de A.; CARDOSO, E. L. On the influence of local and global stress constraint and filtering radius on the design of hinge-free compliant mechanisms. Structural and Multidisciplinary Optimization, v. 58, n. 2, p. 641-655, 2018.

PEREIRA, J. T.; FANCELlO, E. A.; BARCELLOS, C. S. Topology optimization of continuum structures with material failure constraints. Structural and Multidisciplinary Optimization, v. 26, n. 1-2, p. 50-66, 2004.

PICELLI, R. et al. Stress-based shape and topology optimization with the level set method. Computer Methods in Applied Mechanics and Engineering, v. 329, p. $1-23,2018$.

PILKEY, W. D. Peterson's stress concentration factors. 2nd. ed. New York: John Wiley \& Sons, 2007. 
RAO, S. S. Engineering Optimization: Theory and Practice. 4th. ed. New Jersey: Wiley, 2009.

ROSS, S. A First Course in Probability. 8th. ed. Upper Saddle River, New Jersey: Prentice Hall, 2010.

SANTOS, R. B. dos; LOPES, C. G.; NOVOTNY, A. A. Structural weight minimization under stress constraints and multiple loading. Mechanics Research Communications, v. 81, p. 44-50, 2017.

SANTOS, R. B. dos; TORII, A. J.; NOVOTNY, A. A. Reliability-based topology optimization of structures under stress constraints. International Journal for Numerical Methods in Engineering, v. 114, n. 6, p. 660-674, 2018.

SHARMA, A.; MAUTE, K. Stress-based topology optimization using spatial gradient stabilized xfem. Structural and Multidisciplinary Optimization, v. 57, n. 1, p. $17-38,2018$.

SIGMUND, O. Design of Material Structures using Topology Optimization. 1994. Tese (Ph. D. Thesis) — Technical University of Denmark, Lyngby, Denmark, 1994.

SIGMUND, O. Morphology-based black and white filters for topology optimization. Structural and Multidisciplinary Optimization, v. 33, n. 4-5, p. 401-424, 2007.

SIGMUND, O. Manufacturing tolerant topology optimization. Acta Mechanica Sinica, v. 25, n. 2, p. 227-239, 2009.

SIGMUND, O.; MAUTE, K. Topology optimization approaches. Structural and Multidisciplinary Optimization, v. 48, n. 6, p. 1031-1055, 2013.

SIGMUND, O.; PETERSSON, J. Numerical instabilities in topology optimization: A survey on procedures dealing with checkerboards, mesh-dependencies and local minima. Structural optimization, v. 16, n. 1, p. 68-75, 1998.

SILVA, G. A. da. Otimização topológica de estruturas contínuas considerando incertezas. 2016. Dissertação (Dissertação de Mestrado) — Universidade do Estado de Santa Catarina, Joinville, Brasil, 2016.

SILVA, G. A. da; BECK, A. T. Reliability-based topology optimization of continuum structures subject to local stress constraints. Structural and Multidisciplinary Optimization, v. 57, n. 6, p. 2339-2355, 2018.

SILVA, G. A. da; BECK, A. T.; CARDOSO, E. L. Topology optimization of continuum structures with stress constraints and uncertainties in loading. International Journal for Numerical Methods in Engineering, v. 113, n. 1, p. 153-178, 2018.

SILVA, G. A. da; BECK, A. T.; SIGMUND, O. Stress-constrained topology optimization considering uniform manufacturing uncertainties. Computer Methods in Applied Mechanics and Engineering, v. 344, p. 512-537, 2019.

SILVA, G. A. da; CARDOSO, E. L. Stress-based topology optimization of continuum structures under uncertainties. Computer Methods in Applied Mechanics and Engineering, v. 313, p. 647-672, 2017. 
SILVA, G. A. da; CARDOSO, E. L.; BECK, A. T. Non-probabilistic robust continuum topology optimization with stress constraints. Structural and Multidisciplinary Optimization, 2018.

STEFANOU, G. The stochastic finite element method: Past, present and future. Computer Methods in Applied Mechanics and Engineering, v. 198, n. 9-12, p. 1031-1051, 2009.

STOLPE, M.; SVANBERG, K. An alternative interpolation scheme for minimum compliance topology optimization. Structural and Multidisciplinary Optimization, v. 22, n. 2, p. 116-124, 2001.

SVANBERG, K. The method of moving asymptotes - a new method for structural optimization. International Journal for Numerical Methods in Engineering, v. 24, n. 2, p. 359-373, 1987.

SVÄRD, H. Interior value extrapolation: a new method for stress evaluation during topology optimization. Structural and Multidisciplinary Optimization, v. 51, n. 3, p. $613-629,2015$.

THORE, C.-J. On a nash game for topology optimization under load-uncertainty: Finding the worst load. In: VII European Congress on Computational Methods in Applied Sciences and Engineering. Crete Island, Greece: ECCOMAS Congress 2016, 2016. p. 5-10.

THORE, C.-J.; HOLMBERG, E.; KLARBRING, A. A general framework for robust topology optimization under load-uncertainty including stress constraints. Computer Methods in Applied Mechanics and Engineering, v. 319, p. 1-18, 2017.

TOOTKABOnI, M.; ASADPOURE, A.; GUEST, J. K. Topology optimization of continuum structures under uncertainty - a polynomial chaos approach. Computer Methods in Applied Mechanics and Engineering, v. 201-204, p. 263-275, 2012.

TROYA, M. A. S. de; TORTORELLI, D. A. Adaptive mesh refinement in stressconstrained topology optimization. Structural and Multidisciplinary Optimization, v. 58, n. 6, p. 2369-2386, 2018.

TU, J.; CHOI, K. K.; PARK, Y. H. A new study on reliability-based design optimization. Journal of Mechanical Design, v. 121, n. 4, p. 557-564, 1999.

WANG, C.; QIAN, X. Heaviside projection-based aggregation in stress-constrained topology optimization. International Journal for Numerical Methods in Engineering, v. 115, n. 7, p. 849-871, 2018.

WANG, F.; LAZAROV, B. S.; SIGMUND, O. On projection methods, convergence and robust formulations in topology optimization. Structural and Multidisciplinary Optimization, v. 43, n. 6, p. 767-784, 2011.

WANG, M. Y.; WANG, X.; GUO, D. A level set method for structural topology optimization. Computer Methods in Applied Mechanics and Engineering, v. 192, n. 1-2, p. 227-246, 2003. 
XU, S.; CAI, Y.; CHENG, G. Volume preserving nonlinear density filter based on heaviside functions. Structural and Multidisciplinary Optimization, v. 41, n. 4, p. 495-505, 2010.

YOUN, B. D.; CHOI, K. K. An investigation of nonlinearity of reliability-based design optimization approaches. Journal of Mechanical Design, v. 126, n. 3, p. 403-411, 2003.

YOUN, B. D.; CHOI, K. K.; PARK, Y. H. Hybrid analysis method for reliability-based design optimization. Journal of Mechanical Design, v. 125, n. 2, p. 221-232, 2003.

YOUN, B. D. et al. Reliability-based design optimization for crashworthiness of vehicle side impact. Structural and Multidisciplinary Optimization, v. 26, n. 3-4, p. 272-283, 2004. 